\title{
Retrospective analysis of permanent prostate implants based on patient-specific Monte Carlo dose calculations
}

by

\section{Ali-Reza Mehan Haidari}

\begin{abstract}
A thesis submitted to the Faculty of Graduate and Postdoctoral Affairs in partial fulfillment of the requirements for the degree of

\author{
Master of Science
}

in
\end{abstract}

Physics

Specialization in Medical Physics

Ottawa-Carleton Institute for Physics

Department of Physics

Carleton University

Ottawa, Ontario, Canada

September 2016 


\section{Abstract}

Current dose calculations for permanent prostate implant brachytherapy are performed using the American Association of Physicists in Medicine TG-43 formalism, where dose distributions are derived for a homogeneous water phantom in the absence of inter-seed effects. Using a model-based approach, this study derives more accurate patient models to investigate differences in dosimetry, radiobiological endpoints, and clinical outcomes, compared to the clinical TG-43 based approach.

Post-implant CT images are used to derive unique virtual patient phantoms (both water- and tissue-based) for a cohort of patients. With the use of the Monte Carlo (MC) approach to dose calculations, radiation transport and dose deposition is simulated in these virtual phantoms. Dose and volume metrics are extracted for all the patients and comparisons between the tissue- and water-based approaches are made. Using dosimetric quantities extracted from the two approaches, radiobiological indices are computed and compared. Investigation of clinical outcomes is also performed using a database of patient follow-up data and the computed dosimetric quantities.

Dosimetric quantities are shown to vary between the tissue- and water-based approaches for the prostate and various organs-at-risk, with dose overestimation of up to $9.12 \%$ in the prostate using the water-based approach (TG-43 formalism). This suggests that TG-43 based dose calculations are inaccurate, emphasizing the need for more accurate, model-based approaches to dose calculations. 


\section{Acknowledgments}

I would like to thank my supervisors, Dr. Rowan Thomson and Dr. Joanna Cygler, for all their insight, support, and expertise over the past two years, always providing guidance and suggestions in a positive manner. Both of them have shown passion, enthusiasm, and commitment towards scientific research and their students.

I would like to thank my colleagues and peers at Carleton University for their fruitful discussion and assistance, particularly: Nelson Miksys, Martin Martinov, Patty Oliver, and Stephen Deering.

I would like to express my deepest gratitude towards my family for their unconditional support throughout my studies; without them, none of this would be possible.

Funds for this research were provided by: Robert L. Clarke Graduate Scholarship in Medical Physics, Early Researcher Award from Ministry of Research and Innovation, and the Canadian Cancer Society Research Institute. 


\section{Statement of originality}

This thesis is a summary of the most significant contributions of the author's research while enrolled in the MSc program at Carleton University. Dr. Rowan Thomson and Dr. Joanna Cygler co-supervised the project, providing input on all aspects of the study. Dr. Oliver Holmes (The Ottawa Hospital Cancer Centre) extracted follow-up data from patient medical records for the patient outcomes study. Otherwise, the author of this thesis is responsible for development of methodology, computational work, and data analysis.

\section{Paper in preparation}

A-R. M. Haidari, N. Miksys, J. E. Cygler, O. Holmes, G. Perry, C. E, R. M. Thomson. Retrospective analysis of permanent prostate implants based on Monte Carlo dose calculations.

- Concise summary of the results from this thesis.

\section{Conference abstracts}

A-R. M. Haidari, N. Miksys, J. E. Cygler, C. E, G. Perry, O. Holmes, R. M. Thomson. Effect of Tissue Heterogeneity and Interseed Effects on Urethral Dose for Permanent Prostate Brachytherapy. Brachytherapy 15 (2016): S186-S187.

- Presented as a poster at the 2016 World Congress of Brachytherapy in San Francisco, CA. 


\section{Contents}

$\begin{array}{ll}\text { Abstract } & \text { ii }\end{array}$

$\begin{array}{ll}\text { Acknowledgments } & \text { iii }\end{array}$

Statement of originality iv

Table of contents

List of tables $\quad$ vii

$\begin{array}{ll}\text { List of figures } & \text { ix }\end{array}$

List of acronyms, notation, and definitions $\quad \mathbf{x}$

1 Introduction 1

1.1 Prostate Carcinoma and Brachytherapy . . . . . . . . . . . . . . 1

1.2 Dose Calculations . . . . . . . . . . . . . . . . . . 2

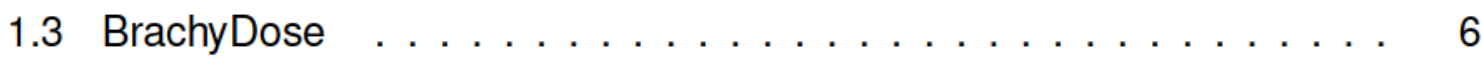

1.4 Radiobiological Modeling . . . . . . . . . . . . . . . . 7

1.5 Thesis Outline . . . . . . . . . . . . . . . . . . . . 10

2 Methods 12

2.1 Patient Cohort Demographics . . . . . . . . . . . . . . . . . . . . . 12

2.2 Derivation of Virtual Patient Phantoms . . . . . . . . . . . . . . . . . . 12

2.2.1 Metallic Artifact Reduction . . . . . . . . . . . . . . 13

2.2 .2 Contour Interpolation . . . . . . . . . . . . . . 16

2.2.3 Tissue Assignment Scheme . . . . . . . . . . . . . . . . 17

2.2.4 Tissue Assignment and Bladder Contrast Agent . . . . . . 20

2.3 Dose Calculations with BrachyDose . . . . . . . . . . . . . . . 21

2.4 Dosimetric Analysis . . . . . . . . . . . . . . . . . . . . . 22 
2.5 Radiobiological Analysis . . . . . . . . . . . . . . . 23

2.6 Outcomes Study . . . . . . . . . . . . . . . . . . . . . 25

3 Results 27

3.1 Dosimetry . . . . . . . . . . . . . . . . . . . 27

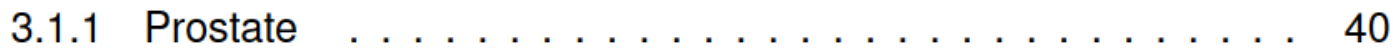

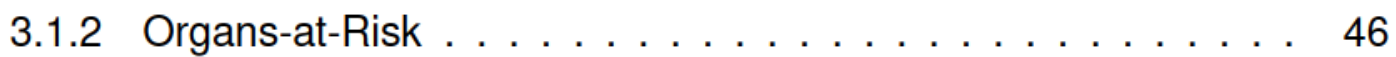

3.2 Radiobiological Modeling . . . . . . . . . . . . . . . . . . . . . . . 49

3.3 Outcome Study . . . . . . . . . . . . . . . . . . . . 52

4 Discussion $\quad 58$

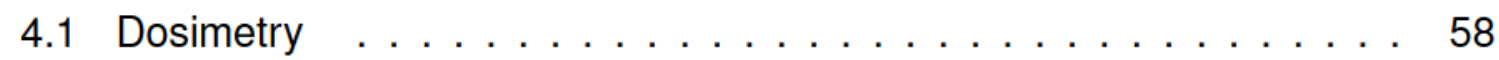

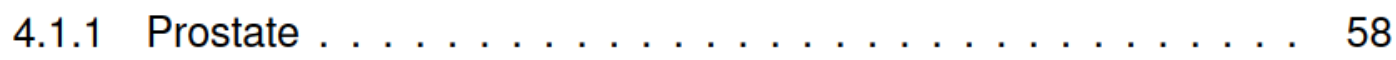

4.1 .2 Organs-at-Risk . . . . . . . . . . . . . . . 63

4.1 .3 General Comments . . . . . . . . . . . . . . . . . . . . 65

4.2 Radiobiological Modeling . . . . . . . . . . . . . . . . 67

4.3 Outcomes Study . . . . . . . . . . . . . . . . . . 68

5 Conclusions and Future Outlook 70

$\begin{array}{ll}\text { Appendices } & 72\end{array}$

$\begin{array}{ll}\text { A Patient Data Storage } & 72\end{array}$

B Philips Brilliance CT Big Bore Calibration Curve 74

$\begin{array}{ll}\text { C Photon Cross-sections } & 75\end{array}$ 


\section{List of Tables}

2.1 Patient and tumor factor demographics . . . . . . . . . . . . . 13

2.2 Elemental composition and mass density of tissues . . . . . . . . 20

2.3 Statistical uncertainties for simulations . . . . . . . . . . . . 21

2.4 Radiobiological modeling parameters $\ldots \ldots \ldots \ldots$

3.1 Absolute and relative organ dose-volume metrics for example patients 28

3.2 Absolute and relative dose-volume metrics for patient cohort . . . . 39

3.3 Toxicity outcomes for patient cohort . . . . . . . . . . . . . . . 53

B.1 CT number to mass density calibration curve $\ldots \ldots \ldots 74$ 


\section{List of Figures}

1.1 Diagram showing coordinate system used in the TG-43 formalism . . 3

2.1 Intermediary steps for derivation of patient models . . . . . . . . . . . 14

2.2 Flowchart of process involved in derivation of patient models . . . . 15

2.3 Mass-energy absorption coefficients of tissues normalized to water . . 18

2.4 Mass attenuation coefficients of tissues normalized to water . . . . . . 19

3.1 Dose distribution and line profiles for example Patient 1 (only ISA) . . 29

3.2 Dose distribution and line profiles for example Patient 1 (only Tissue

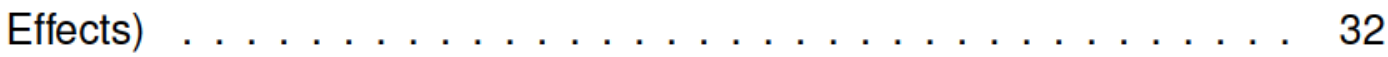

3.3 Dose distribution and line profiles for example Patient $1 \ldots \ldots 4$

3.4 Dose distribution and line profiles for example Patient $2 \ldots \ldots$

3.5 Dose distribution and line profiles for example Patient $3 \ldots \ldots$

3.6 Histogram of distribution of prostate dose metrics . . . . . . . . . . 41

3.7 Histogram of distribution of prostate volume metrics . . . . . . . . . 42

3.8 Boxplot of normalized prostate dose-volume metrics . . . . . . . . 43

3.9 Normalized prostate metrics as a function of calcification in prostate . 45

3.10 Boxplot of the distribution of absolute OAR dose-volume metrics . . . 47

3.11 Boxplot of the distribution of normalized OAR dose-volume metrics . . 48

3.12 Histogram of BED and TCP for 110 Gy cut-off . . . . . . . . . . . 50 
3.13 Cumulative DVH illustrating discarded voxels with 110 Gy cut-off . . . 51

3.14 Histogram of BED and TCP for 9.43 Gy cut-off . . . . . . . . . . 53

3.15 Radiation toxicity outcomes related to absolute dose metrics . . . . 54

3.16 Radiation toxicity outcomes related to absolute volume metrics . . . . 56

3.17 Radiation toxicity outcomes related to normalized dose-volume metrics 57

C.1 Photon cross-sections for various tissues $\ldots \ldots \ldots \ldots$ 


\section{List of acronyms, notation, and definitions}

\section{Notation}

$\alpha$

$\beta$

$\eta$

$\frac{\mu_{e n}}{\rho}$

$\underline{\mu}$

$D_{R x}$

$D_{t}$

$D_{x}$

$D_{x c c}$

$M C$

$M C_{\text {noISA }}$

$T_{\text {eff }}$

$T G 43$

$V_{x}$

\section{Acronyms}

Coefficient describing single-strand DNA breakage

Coefficient describing double-strand DNA breakage

Percentage of voxels in the prostate region assigned to calcifications

Mass-energy absorption coefficient

Mass attenuation coefficient

Prescription dose, 145 Gy for ${ }^{125}$ I implants

Minimum dose threshold for low-dose inclusions in the simple radiobiological model

Minimum dose received by $x$ percent of the volume for the relevant structure

Minimum dose received by $x$ volume of structure in cubic centimeters Virtual patient models with heterogeneous tissue assignment scheme and inter-seed effects enabled

Virtual patient models with heterogeneous tissue assignment scheme and inter-seed effects disabled

Radiobiologically effective treatment time, time when rate of cell death is equivalent to rate of cell proliferation

Virtual patient models with homogeneous (water) tissue assignment scheme and inter-seed effects disabled

Fractional organ volume that receives at least $x$ percent of the prescription dose $D_{R x}$

American Association of Physicists in Medicine, scientific and professional organization of medical physicists 
BED Biologically Effective Dose, quantity to describe tissue-specific biological response to radiation damage

CPE Charged Particle Equilibrium, condition whereby the number of charged particles leaving a volume for each energy and particle type equals the number entering the volume

CT Computed Tomography, imaging technique that uses $\mathrm{X}$-rays to produce body images

CTCAE Common Terminology Criteria for Adverse Events, set of criteria for the standardized classification of adverse effects due to cancer treatments

DFS Disease Free Survival, length of time after primary cancer treatment where the patient survives and shows no signs or symptoms of cancer

DICOM-RT Digital Imaging and Communications in Medicine for Radiotherapy, standardized file format for treatment and imaging data

DSF Dose Scaling Factor, used to convert dose per history to absorbed dose for each voxel

DVH Dose-Volume Histogram, binned absorbed dose to fractional organ volume receiving a minimum of said dose

HU Hounsfield Unit, scale for describing radiodensity

ICRP International Commission on Radiological Protection, international organization that provides recommendations and guidance for radiation protection

ICRU International Commission on Radiation Units and Measurements, international organization that provides recommendations on radiation related quantities and units

Kerma Kinetic Energy Released per Unit Mass, sum of initial energies of all charged particles released by uncharged ionizing radiation 
MAR

MBDCA

MC

MRI

NCCN

$\mathrm{NCl}$

OAR

PIPB

PSA

SPECT

STR

TAS

TCP

TG-43
Metallic Artifact Reduction, technique used to mitigate metallic artifacts present in post-implant CT images

Model-Based Dose Calculation Algorithm, dose calculation algorithm that utilizes detailed heterogeneous patient models for computation of dose distributions

Monte Carlo, the Monte Carlo technique for computation of dose distributions

Magnetic Resonance Imaging, imaging technique that uses magnetic fields and radio waves to form images of the body

National Comprehensive Cancer Network

National Cancer Institute

Organs At Risk, organs in close proximity to the prostate region that do not require treatment, including the bladder, rectum, and urethra Permanent Implant Prostate Brachytherapy, procedure whereby encapsulated radionuclides are implanted into a patients prostate Prostate-Specific Antigen, protein produced by prostate gland that is typically elevated for patients with prostate cancer Single-Photon Emission Computed Tomography Simple Threshold Replacement, algorithm for the mitigation of metallic artifacts

Tissue Assignment Scheme, set of rules that define tissue compositions for each voxel of the image

Tumor Control Probability, probability of tumor eradication under the constraints of the radiobiological model

AAPM Task Group 43 Report, brachytherapy dose calculation formalism 
TNM

\section{Definitions}

Seeds Encapsulated Radionuclides

Phantom Virtual Patient Model, computation patient phantom used for computation of dose distributions with BrachyDose

Voxel Three-Dimensional Pixel 


\section{Chapter 1}

\section{Introduction}

This thesis presents a retrospective dosimetric study for 157 patients who underwent lowdose rate permanent implant prostate brachytherapy (PIPB) at The Ottawa Hospital Cancer Centre (Ottawa, Canada). This introduction sets the stage for concepts related to dosimetry, radiobiological modeling, and outcomes analysis that will be reported and discussed in this study.

\subsection{Prostate Carcinoma and Brachytherapy}

Prostate carcinoma is the most common cancer, while being the third most common cause of cancer death, among Canadian men. Over 24,000 of the 100,500 (23.9\%) new male cancer cases in 2015 have been attributed to prostate cancer ${ }^{1}$. According to the National Comprehensive Cancer Network (NCCN), adenocarcinoma, the most common form of prostate cancer, can be graded according to three factors ${ }^{2}$ : TumorNode-Metastasis (TNM) staging (describes the tumor size and confinement within the prostate, regional lymph node metastasis, and distant metastasis); Gleason Sum Score (GS) staging (describes the growth of the cancer cell pathologically, based on biopsy samples); and Prostate Specific Antigen (PSA) level (a protein produced by cells in the prostate gland that can be measured with a blood test; elevated levels are present in men with prostate cancer). ${ }^{3}$ The NCCN classification scheme is as 
follows:

- Low-risk: T-stage $\leq 2 \mathrm{~b}, \mathrm{GS} \leq 6$, and PSA $\leq 10$.

- Intermediate-risk: T-stage $\leq 2 \mathrm{~b}$ and/or GS $=7$ and/or $10<\mathrm{PSA} \leq 20$.

- High-risk: T-stage $=2 \mathrm{c}$, or GS $\geq 8$, or PSA $>20$.

Patients with low or intermediate-risk prostate carcinoma are recommended ${ }^{3}$ to receive brachytherapy as monotherapy, or as hybrid therapy (brachytherapy in conjunction with external beam radiation therapy). Prostate brachytherapy is an outpatient radiotherapy technique where encapsulated radioactive sources (seeds) are implanted, either permanently or temporarily, into the target through the perineum under guidance of a transrectal ultrasound probe. Seed locations are optimized through an inverse planning technique where dose to the tumor is maximized while sparing dose to physician-contoured organs-at-risk (OAR). The advantage to this technique is that it allows for a highly conformal radiation treatment of the tumor from within the prostate volume, reducing radiation to critical OAR. For this study, patients received a low-dose rate ( $\leq 2 \mathrm{~Gy} \mathrm{~h}^{-1}$ ) permanent implant prostate brachytherapy (PIPB) treatment with an ${ }^{125}$ | radionuclide, either as monotherapy or hybrid therapy.

\subsection{Dose Calculations}

Currently, the clinical standard for calculating dose (energy absorbed per unit mass of medium) distributions for brachytherapy treatments is outlined in the American Association of Physicists in Medicine (AAPM) Task Group 43 report (TG-43) ${ }^{4}$. According to this formalism, the dose rate for a single radioactive source is derived using the following expression:

$$
\dot{D}(r, \theta)=S_{K} \cdot \Lambda \cdot \frac{G_{L}(r, \theta)}{G_{L}\left(r_{0}, \theta_{0}\right)} \cdot g_{L}(r) \cdot F(r, \theta)
$$


for a two-dimensional line source approximation, where: $S_{K}$ is the air-kerma (kinetic energy released per unit mass) strength (air-kerma rate in vacuo at a given distance multiplied by the square of the distance); $\Lambda$ is the dose-rate constant in water (ratio of the dose rate at a reference point $P\left(r_{0}, \theta_{0}\right)$ to $S_{K}$, where $r_{0}=1 \mathrm{~cm}$ and $\left.\theta_{0}=90^{\circ}\right) ; G_{L}$ is the geometry function for a line source (which gives the variation of dose rate due to the spatial distribution of radioactivity within the source, neglecting any attenuation or scattering effects); $g_{L}$ is the radial dose function (which takes into account the effects of attenuation and scattering on the dose rate as a function of distance); $F$ is the anisotropy function (which describes the angular variation of dose rate). Figure 1.1 illustrates the coordinate system used in the TG-43 formalism.

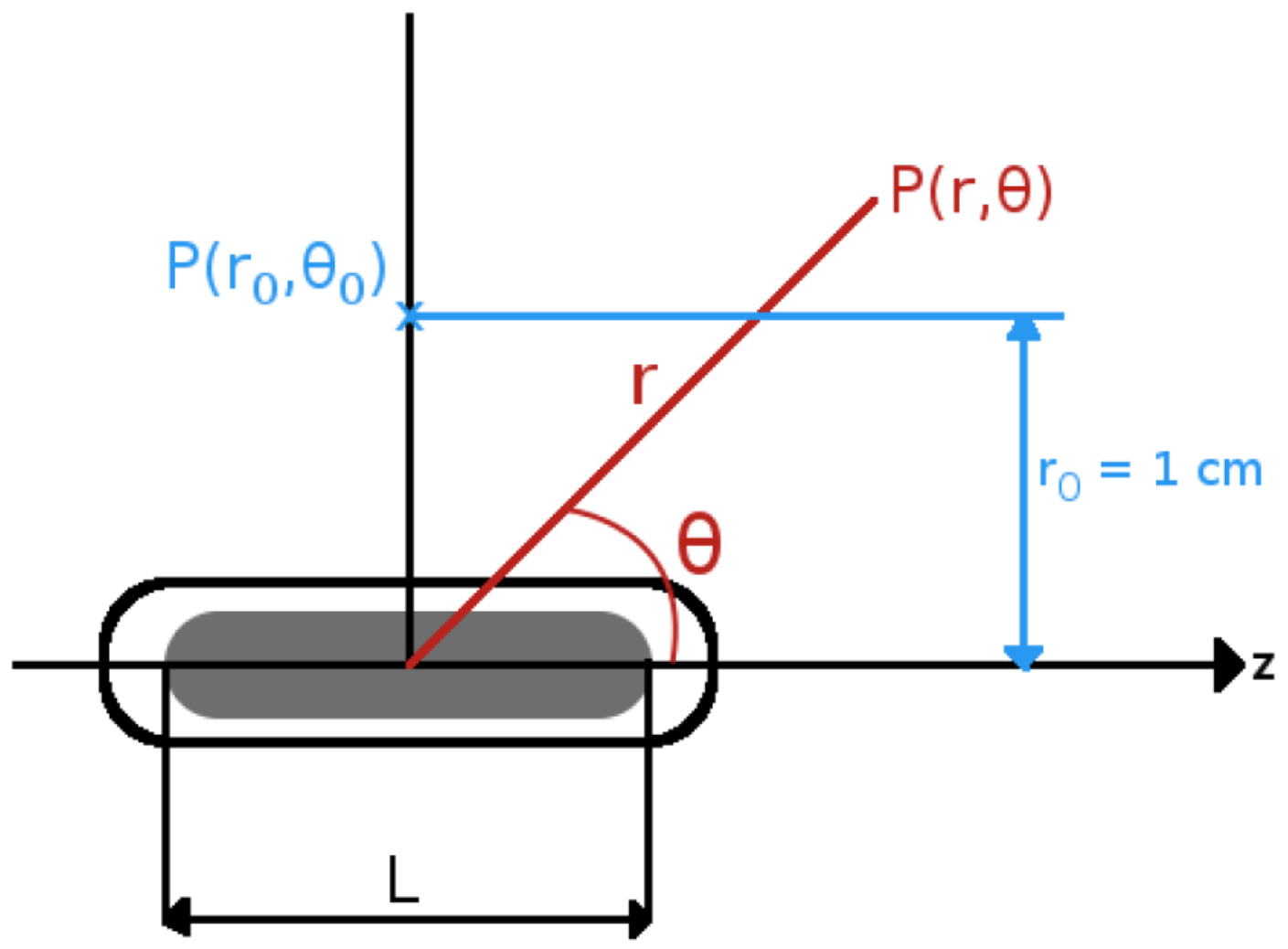

Figure 1.1: Diagram showing coordinate system used in the TG-43 formalism ${ }^{4}$ with single radioactive source (seed) shown.

According to the AAPM TG-43 protocol, the dose distribution for each patient is 
calculated under two assumptions: the patient is made up of an infinitely expansive water medium, ignoring tissue heterogeneities present in the patient anatomy; the dose contribution of each seed is independent of any other seed, ignoring inter-seed scattering and attenuation effects among neighbouring seeds. These two assumptions provide a simplistic view of the patient anatomy and radiation transport within this anatomy. When considering the energies relevant to prostate brachytherapy (e.g. average photon energy of ${ }^{125} \mathrm{I}$ is $28 \mathrm{keV}$ ), photon interactions are dominated by the photoelectric effect that has a cross section of interaction per atom proportional to $\frac{Z^{4}}{(h \nu)^{3}} 5$, where $Z$ is the atomic number of the medium, and $h \nu$ is the photon energy. As a result of this dependence on the atomic number of the medium, any deviations of effective atomic number, $Z_{\text {eff }}$, of the tissue relative to water can affect the probability of photon interaction, and therefore alter dose distributions. In the case of prostate brachytherapy, patients with large accumulations of calcium $(Z=20)$ salts (calcifications) within the prostate region may have areas of under-dosage (cold spots) due to the attenuation of photon fluence (number of particles $d N$, incident on a sphere of cross-sectional area $d a$ ) and lack of backscatter that is not taken into account in the AAPM TG-43 paradigm.

Recently, AAPM-ESTRO-ABG-ABS TG- $186^{6}$ advised for the use of advanced model-based dose calculation algorithms (MBDCA) for brachytherapy, which include taking into consideration tissue heterogeneities and inter-seed effects. Model-based approaches involve simulating radiation transport in virtual phantoms (models) that have heterogeneous media (various tissues and sources) as opposed to being water homogeneously. Current model-based approaches to dose calculations for brachytherapy applications include collapsed-cone superposition/convolution ${ }^{7-10}$, grid-based Boltzmann equation solvers ${ }^{11,12}$, and Monte Carlo simulations ${ }^{13-15}$. Collapsed-cone superposition/convolution method involves using direct raytracing of the primary photons to compute primary dose to water using the kerma approximation. Dose to water 
from first scatter, and multiple scatter, originating from raytracing results of the primary dose are computed. Primary and scattered doses are scaled to account for heterogeneities using similar raytracing operations ${ }^{9,16}$. While being computationally fast, this method is limited in accuracy and breadth of heterogeneity corrections ${ }^{17}$. Grid-based Boltzmann equation solvers use deterministic methods for solving the linear Boltzmann transport equation (LBTE), usually through spatial discretization ${ }^{18}$. Solutions closest to the "truth" are dependent on the amount of discretization, resulting in high computational intensity ${ }^{17-19}$ for this method. Monte Carlo simulations involve random sampling of particles to solve the LBTE. Absorbed dose per voxel (three-dimensional pixel) is typically scored using a track-length estimator based on the assumption of absorbed dose and collisional kerma equivalency under conditions of charged-particle equilibrium (CPE, the number of charged particles leaving a volume for each energy and particle type equals the number entering). In this study, the MC method for dose calculations is used for computing absorbed dose in patient models.

Using the MC method of MBDCA in this study, models are derived from postimplant CT images using a tissue assignment scheme (TAS), a set of rules that define tissue compositions for each voxel of the image. Physician-defined contours delineate organ volumes that are assigned tissue compositions taken from literature ${ }^{20-22}$. CT numbers (in Hounsfield Units, HU) relating to mass densities (see Appendix B) are used to discern various tissues present within or outside organ volumes, and assign voxel-specific mass densities. Pre-modeled seeds are overlaid onto virtual phantoms at dosimetrist-defined seed centers. Radiation transport is performed in heterogeneous medium $(m)$ with absorbed dose to local medium $\left(D_{m, m}\right)$ being reported alongside traditional TG-43 water $(w)$ values $\left(D_{w, w}\right)$.

Work in the literature by Carrier et al. ${ }^{23},{ }^{24}$, Fekete et al. ${ }^{25}$, Chibani et al. ${ }^{26}$, 
Miksys et al. ${ }^{27}$, Mason et al. ${ }^{28}$ making use of these model-based approaches have shown overestimation of the minimum dose received by $90 \%$ of the prostate volume $\left(D_{90}\right)$ of up to $14.1 \%$ by the AAPM TG-43 dose calculation method, making it imperative to consider tissue heterogeneities and inter-seed effects when doing post-implant dosimetry.

\subsection{BrachyDose}

Monte Carlo (MC) simulations are a state-of-the-art approach for simulating radiation transport and calculating dose for brachytherapy treatments. Using the EGSnrc (Electron Gamma Shower) ${ }^{29,30}$ user-code for fast dose calculations for brachytherapy, BrachyDose ${ }^{14}$ simulates radiation transport and energy deposition of brachytherapy sources in detailed patient models rapidly.

The use of Yegin's multi-geometry package ${ }^{31}$ allows for complex geometries to be modeled using a combination of rectilinear, cylindrical, spherical, and conical shapes. BrachyDose allows for pre-modeled sources from a seed database ${ }^{32}$ to be superimposed onto unique virtual patient phantoms (.egsphants, discussed in 2.2) with the option of turning inter-seed effects on and off.

BrachyDose simulates radiation transport and scores dose for each voxel in a virtual patient phantom using a tracklength estimator ${ }^{14}$. This is done under the assumption that the absorbed dose is equal to the collisional kerma (kinetic energy released per unit mass, the energy transferred to a charged particle that undergoes an ionizing interaction in the medium) since conditions of CPE are satisfied. Given the low energy nature of the photons being emitted by the seeds, the ranges of the electrons produced $(0.013 \mathrm{~mm}$ for $25 \mathrm{keV}$ electrons in water) are less than any voxel dimension (approximately $0.3 \mathrm{~mm} \times 0.3 \mathrm{~mm} \times 2 \mathrm{~mm}$ ), meaning the energy of electrons is deposited locally. Therefore, under conditions of CPE, dose $D$, is equal to 
collisional kerma $K_{c o l}$, given by the following expression (for a monoenergetic photon beam):

$$
D \stackrel{C P E}{=} K_{c o l}=E \cdot \phi \cdot\left(\frac{\mu_{e n}}{\rho}\right)
$$

where: $E$ is the energy of the photons with a photon fluence $\phi$, and mass-energy absorption coefficient, $\frac{\mu_{e n}}{\rho}$, for the medium. Using a tracklength estimator, BrachyDose scores dose $D^{j}$ for the $j^{\text {th }}$ voxel given by the following expression:

$$
D^{j}=\left[\sum_{i} E_{i} t_{i}\left(\frac{\mu_{e n}}{\rho}\right)_{i}\right] / V_{j}
$$

where: $E_{i}$ is the energy for the $i^{t h}$ photon that has a tracklength in the voxel of $t_{i}$; $\left(\frac{\mu_{e n}}{\rho}\right)_{i}$ the mass-energy absorption coefficient for the $i^{t h}$ photon; $V_{j}$ the volume of the $j^{\text {th }}$ voxel.

Relative statistical dose uncertainties for each voxel are estimated based on a history-by-history method ${ }^{33}$, where a history is defined as a single starting particle (photon). If $X_{i}$ is the absorbed dose in a single voxel for an independent history $i$, the uncertainty $s$ on the total dose received by the voxel over the total number of histories $N$, is given by the following expression:

$$
s=\sqrt{\frac{1}{N-1}\left[\frac{\sum_{i=1}^{N} X_{i}^{2}}{N}-\left(\frac{\sum_{i=1}^{N} X_{i}}{N}\right)^{2}\right]}
$$

\subsection{Radiobiological Modeling}

Absorbed dose is a physical quantity describing the amount of energy absorbed per unit mass of medium, but does not describe the biological effect of this dose. Using the linear-quadratic cell inactivation model ${ }^{34}$ that describes the cell survival for a given amount of ionizing radiation, one may describe the surviving fraction of cells, 
$S$, that receive an absorbed dose, $D$, by the following expression:

$$
S=\exp \left(-\left[-\alpha D-\beta D^{2}\right]\right)
$$

and the associated biologically effective dose (BED) that represents tissue-specific biological response to radiation damage:

$$
B E D=-\frac{1}{\alpha} \ln S=D\left[1+\frac{D}{(\alpha / \beta)}\right]
$$

where $\alpha$ describes the intrinsic radiosensitivity of the cells, or the average yield of cell kills resulting from single-strand DNA breaks, while $\beta$ characterizes cell kills resulting from double-strand DNA breaks. Following the method outlined by Dale et al ${ }^{35,36}$, the analytic expression for the BED of prostate implants that use a single type of radionuclide (with half-life $T_{1 / 2}$ and decay constant $\lambda$ ) can be derived by additionally modeling: cell repopulation using the potential doubling time of the tumour cells $\left(T_{p o t}\right)$; and repair of sub-lethal damage ${ }^{3,37}$ (when only one of two critical targets needed for cell inactivation are damaged). It can be shown that the BED for a permanent prostate implant is then given by:

$$
B E D=D\left(T_{e f f}\right) R E\left(T_{e f f}\right)-\ln 2 \frac{T_{e f f}}{\alpha T_{p o t}}
$$

where $D\left(T_{e f f}\right)$ is the total dose within the radiobiologically effective treatment time, $T_{\text {eff }}$ :

$$
T_{\text {eff }}=1.44 T_{1 / 2} \ln \left[\alpha \cdot D \cdot \frac{T_{\text {pot }}}{T_{1 / 2}}\right]
$$

The relative effectiveness at the effective treatment time, $R E\left(T_{\text {eff }}\right)$ is given by:

$$
R E\left(T_{\text {eff }}\right)=1+\left(\frac{\beta}{\alpha}\right) \frac{\dot{D}_{0}}{(\mu-\lambda)} \cdot \frac{1}{1-e^{-\lambda T_{e f f}}}\left\{1-e^{-2 \lambda T_{e f f}}-\frac{2 \lambda}{\mu+\lambda}\left(1-\exp \left[-(\mu+\lambda) T_{e f f}\right]\right)\right\}
$$


where $\dot{D}_{0}$ is the initial dose rate, and $\mu$ the time constant that models sub-lethal damage repair capability. Considering the inhomogenous dose-rate distributions for prostate implants, the BED calculation must be partitioned into subvolumes that are assumed to have a uniform dose-rate. ${ }^{38,39}$ Therefore, the total equivalent uniform biologically effective dose (EUBED) for an implant may be computed using the following expression:

$$
E U B E D=-\frac{1}{\alpha} \ln \left(\sum_{i} v_{i} e^{-\alpha B E D_{i}}\right)
$$

where $v_{i}$ is the fractional volume of the subvolume (voxel volume), and $B E D_{i}$ is the BED of the subvolume. EUBED simplifies to BED for homogeneous dose distributions. EUBED will be referred to as BED herein for simplicity.

The link between amount of radiation-induced cell kill (BED) and clinical response can be modelled under the assumption that cell death follows Poisson statistics; therefore, the tumor control probability (TCP) for permanent prostate implants ${ }^{3,40}$, the probability of no surviving tumor cells, can be computed with the following expression:

$$
T C P=\exp \left[-N_{0} e^{-\alpha \cdot B E D}\right]
$$

where $N_{0}$ is the total number of tumor cells prior to the start of treatment. While this model provides mathematical ease in implementation, it should be noted that it does not take into consideration cell proliferation during the treatment (leads to an underestimation of cure rate $)^{41}$ and improved models have been derived in the literature ${ }^{42,43}$. This study aims to apply this radiobiological model with a voxel-by-voxel approach using advanced MBDCA to compute radiobiological indicies to compare with clinical TG-43 derived parameters. 


\subsection{Thesis Outline}

Dose calculations performed using the current clinical formalism, outlined in the TG43 report, lack any consideration of tissue heterogeneities and inter-seed effects that are shown to significantly alter dose distributions, particularly at the low photon energies used in brachytherapy. With the use of advanced model-based dose calculation algorithms (MBDCA), these effects may be considered, allowing one to quantify and analyze the differences in the dose distributions between the two methods. Applying these advanced MBDCA and drawing dosimetric comparisons involves deriving patient specific models (virtual patient phantoms), simulating radiation transport, and extracting relevant dosimetric quantities for analysis.

Chapter 2 provides an overview of the method employed in the development of patient phantoms from post-implant CT images, exploring the issues while outlining the solutions in the process. Using the same process, two phantoms are derived for each patient: one following the protocol outlined in TG-43, while the other includes tissue heterogeneities and inter-seed effects. Simulation parameters used to compute dose distributions for each model using the Monte Carlo method are also outlined in this chapter. Furthermore, Chapter 2 describes the analysis techniques used to perform a dosimetric, radiobiological, and outcomes study, comparing the two dose calculation approaches.

Chapter 3 begins by showing three example patients used to describe the physical phenomena observed by considering tissue heterogeneities and inter-seed effects on prostate and organs-at-risk (OAR) dose distributions. Results for the entire patient cohort are then presented, along with radiobiological indices, and outcomes studies. Chapter 4 provides a discussion of the results presented in Chapter 3 , framing them in terms of analogous work in the literature. Chapter 5 concludes the results in the study and provides outlook on future works in the field. 
This study is unique in that it applies advanced MBDCA to perform radiobiological modeling and outcomes analysis for a large cohort of patients. It aims to provide insight while outlining current issues in the realm of MBDCA applied to brachytherapy treatments. 


\section{Chapter 2}

\section{Methods}

\subsection{Patient Cohort Demographics}

Table 2.1 presents patient and tumor factor demographics for the 157 patients in this study that were treated with low-dose rate PIPB at The Ottawa Hospital Cancer Centre between November 192007 and October 26 2015. Patients eligible for lowdose rate PIPB undergo a prostate volume study prior to the procedure. At the time of the procedure, treatment plans are generated using an inverse planning method. Patient statistics, tumor factors, treatment toxicity outcomes, and PSA followups were extracted from medical records by radiation oncology resident Dr. Oliver Holmes; patient treatment data were input into an anonymized database and extracted for usage.

\subsection{Derivation of Virtual Patient Phantoms}

Patients that underwent low-dose rate PIPB at The Ottawa Hospital Cancer Center had 30-day post-implant CT imaging performed. Image data, voxel dimensions, dosimetrist-defined seed centers, reference air kerma rate per seed, and physiciandefined organ contours are stored in the Digital Imaging and Communications in 
Table 2.1: Patient and tumor factor demographics for 157 patients included in the study.

\begin{tabular}{cccccc}
\hline \hline \multicolumn{1}{c}{ Age } & & & Gleason Score & {$[\mathrm{N}]$} \\
\cline { 1 - 2 } \cline { 5 - 6 } Mean & 63 & & $\leqslant 6$ & 120 \\
SD & 7 & & 7 & 32 \\
Range & $46-79$ & & $>7$ & 1 \\
Missing & 8 & & Missing & 4 \\
& & & & \\
Pre-treatment PSA & {$[\mathrm{ng} / \mathrm{mL}]$} & & Clinical T-stage & {$[\mathrm{N}]$} \\
\cline { 1 - 2 } \cline { 5 - 6 } Mean & 6.5 & & T1A & 1 \\
SD & 2.5 & & T1C & 129 \\
Range & $0.63-15.4$ & & T2A & 20 \\
$<5$ & 48 & & T2B & 3 \\
$5-10$ & 99 & & Missing & 4 \\
$>10$ & 7 & & & \\
Missing & 3 & & & \\
\hline \hline
\end{tabular}

Medicine for Radiotherapy (DICOM-RT) standard. Using a multi-step Python (Python Software Foundation 2016) script these DICOM-RT files are converted to unique virtual patient phantoms (.egsphants) for each patient to be used with BrachyDose to derive dose distributions, a process outlined in Figures 2.1 and 2.2. The script produces, in batch mode, CT images with overlaid organ contours and seed locations, .egsphant, and .egsinp (BrachyDose input files that include seed locations and various simulation parameters) for a list of patients.

\subsubsection{Metallic Artifact Reduction}

With the implementation of MBDCA it is vital to construct accurate virtual patient phantoms based on post-implant CT images. One problem this poses with brachytherapy is that seed artifacts in the images can lead to large dose errors ${ }^{44-46}$. Metallic artifacts present in the CT images as a result of the implanted seeds need to be corrected for with a simple threshold replacement (STR) technique. While many metallic artifact reduction schemes for brachytherapy have been explored ${ }^{45-47}$ such as fan beam virtual sinogram, 3D median filter, and raw sinogram, simple thresh- 

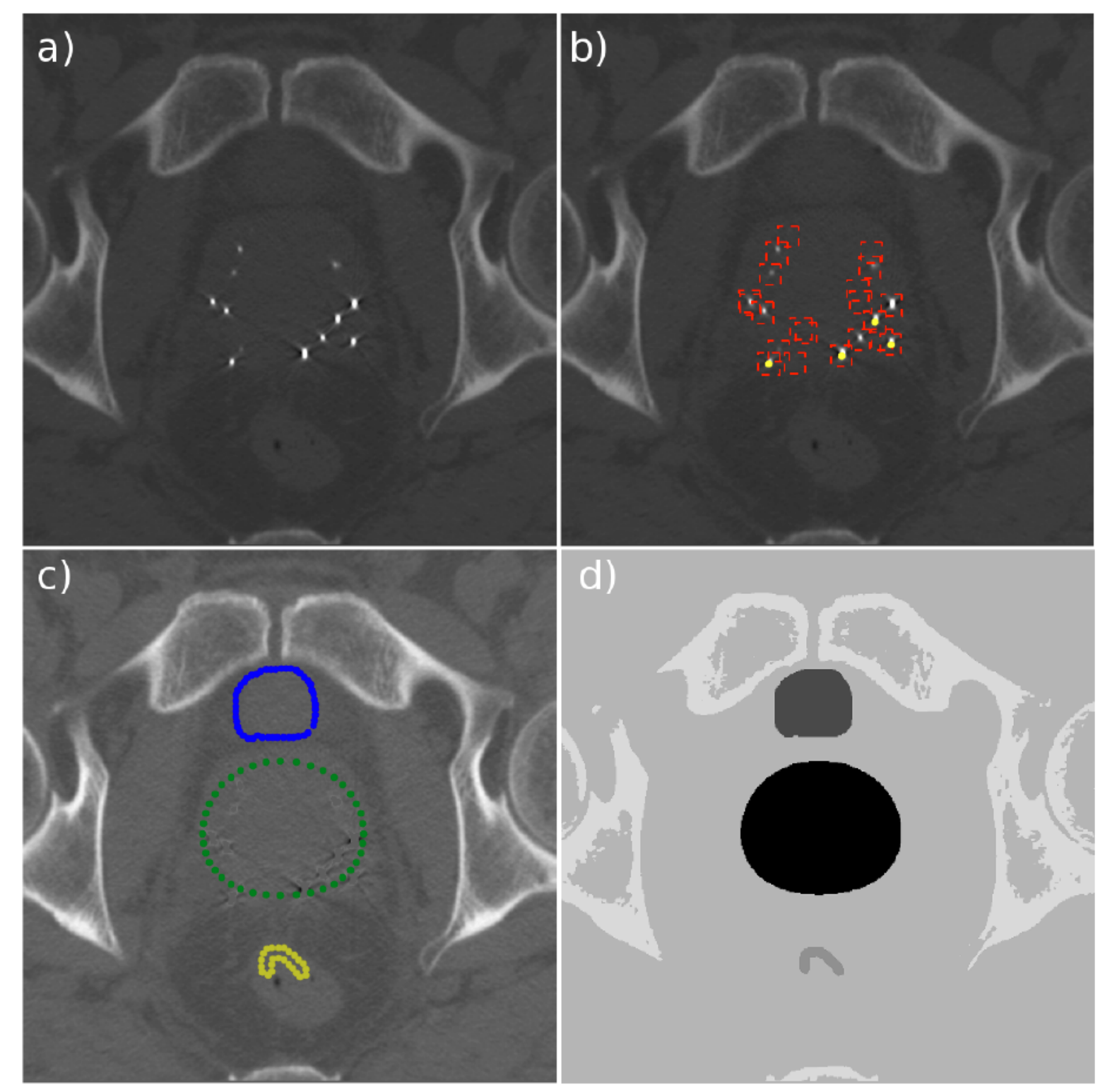

Figure 2.1: Overview of intermediary steps for the creation of virtual patient phantoms (.egsphants): (a) raw post-implant CT image that is extracted from DICOM CT files; (b) CT image with seed centres (yellow dots) and simple threshold replacement (STR) regions (red rectangle) overlaid; (c) corrected CT image with contours for the bladder (blue), prostate (green), and rectum (yellow) overlaid; (d) grayscale virtual patient phantom to be used for simulations with BrachyDose. 


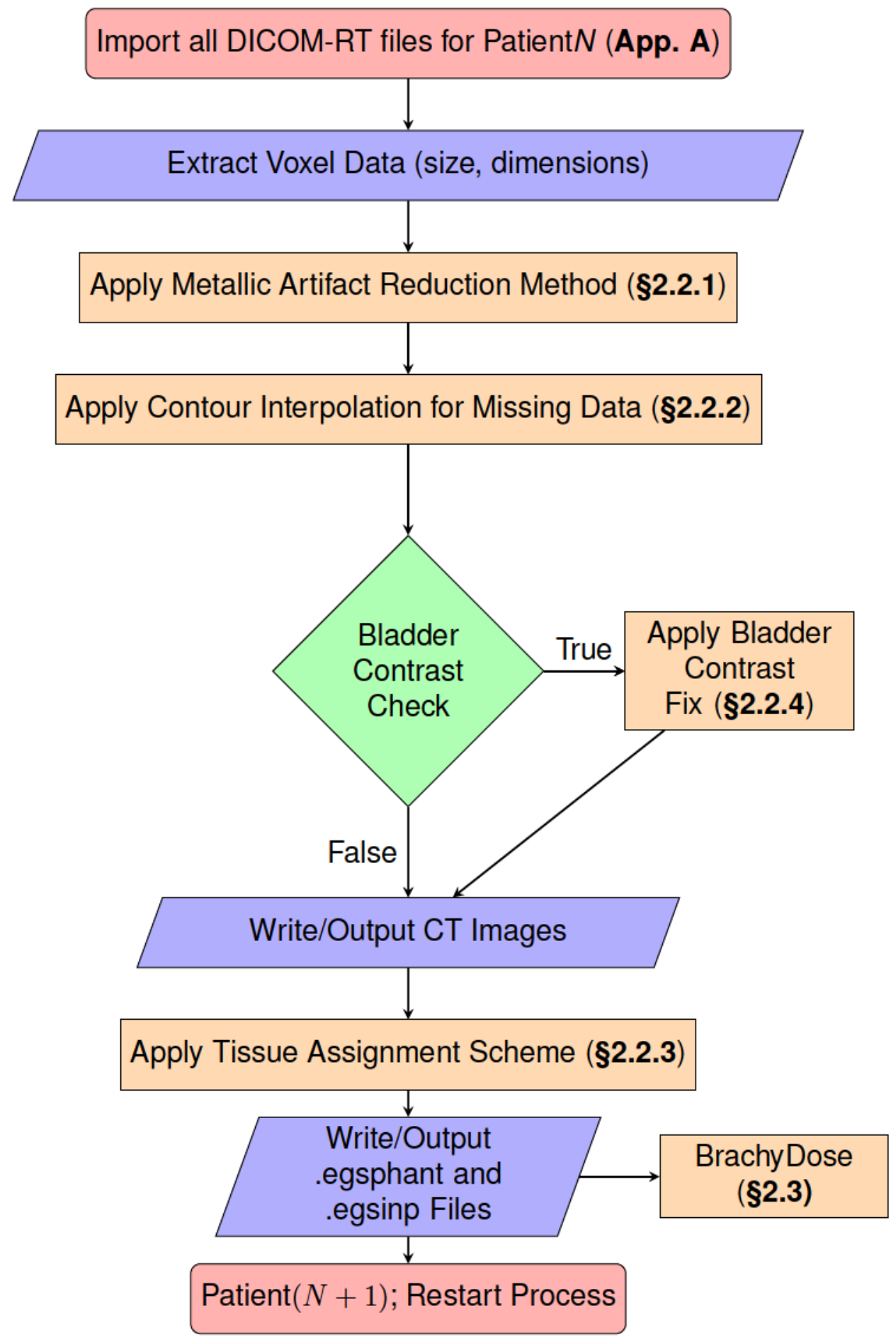

Figure 2.2: Flowchart of in-house python script for developing virtual patient models (.egsphants). Major steps are labeled with relevant section that give detailed explanation of the method used. 
old replacement (STR) provides a fast and effective method of mitigating metallic artifacts.

STR is a technique guided by seed-center locations defined in the DICOM-RT files. It defines a cubic CT volume of $20 \times 20 \times 4$ voxels $(x \times y \times z)$, corresponding to a $6.25 \mathrm{~mm} \times 6.25 \mathrm{~mm} \times 8.0 \mathrm{~mm}$ region (on average) about each seed-center that is flagged for artifact reduction. This constraining of voxels near seed-centers is done to minimize the elimination of high CT numbers that correspond to bone or calcification within the target volume. Furthermore, within this marked volume, voxels with CT numbers greater than a certain threshold (243 HU), corresponding to the upper-bound of soft-tissue CT numbers, are replaced by the inter-patient soft-tissue average $(\mathrm{N}=110)$ inside the prostate $(25 \mathrm{HU})$.

\subsubsection{Contour Interpolation}

For the creation of virtual patient phantoms (.egsphants), physician-defined organ contours were used to delineate organ volumes within the CT images. Any CT slice with missing organ contour information results in unrealistic, discontinuous organ volumes in the virtual patient phantoms. Using a weighted linear interpolation method, missing contour points can be estimated.

For each contoured organ, the first and last CT images with contour information are assumed to be the extremes of the organ in the $z$ direction. Each CT slice contains a set of $x, y$ coordinates corresponding to contour points that encloses a cross-sectional organ area for that slice. Any slice between the two extremes missing a set of coordinates is corrected by finding the closest slices with contour information above and below in the $z$ direction. For each $x, y$ point on the upper CT slice, the closest $x, y$ point on the lower slice is found, where the weighted sum of the individual elements in the coordinate are computed. Weighting is determined by taking the 
inverse of the number of slices between the two extremes. The upper slice (most proximal) is given the heaviest weight since it is assumed the most accurate.

This method works well for prostate brachytherapy treatments in this study since each contoured organ consists of a single continuous area that lacked extreme concavity. For interpolation of more complex contoured areas (i.e. branch contours in lung treatments, or intersecting contours to exclude areas within a larger contour) advanced techniques are available in the literature ${ }^{48-50}$.

\subsubsection{Tissue Assignment Scheme}

Once CT images are corrected for metallic artifacts using STR and all missing contours are corrected, a contour-guided voxel-by-voxel tissue assignment scheme (TAS) is applied. Using this scheme, every voxel in the CT image is assigned an elemental composition (tissue), and mass density, that is dependent on the voxel's image location (in relation to organ contours) and CT number (in Hounsfield Units).

For prostate brachytherapy procedures contoured organs include the prostate, bladder, rectum, and urethra that define image regions. If a voxel is located in any of the image regions a mass density threshold is used to determine tissue assignment. The density of each voxel is determined using a CT number to mass density calibration table (excluding patients with contrast present in their bladders (see 2.2.4) and mass densities less than air) shown in Appendix B.

A single tissue assignment scheme (labeled $M C$ ) applied to the cohort of patients in this study is presented in Table 2.2, with mass-energy absorption, and mass attenuation coefficients of various media relative to water shown in Figure 2.3 and Figure 2.4, respectively. 


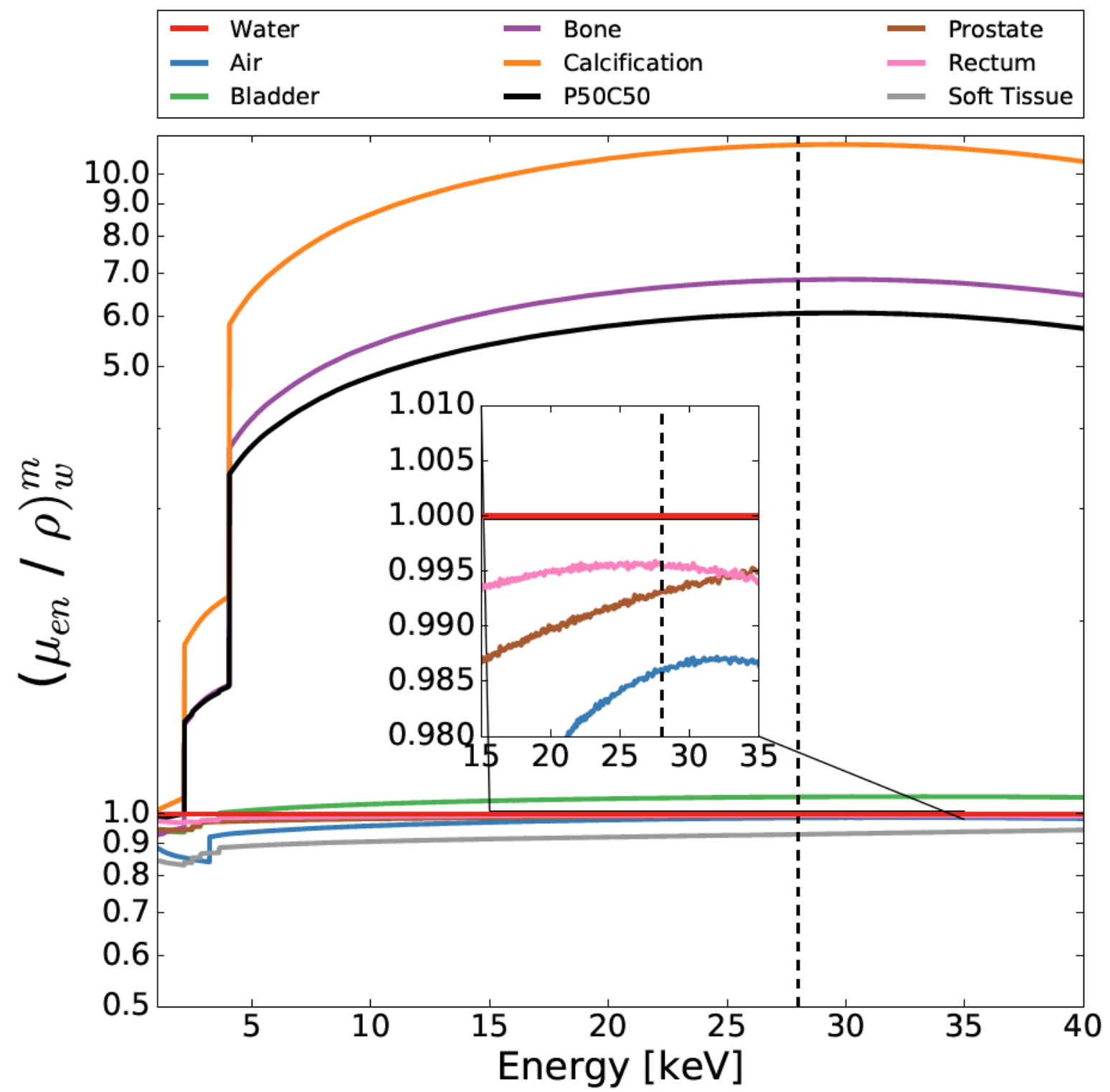

Figure 2.3: Mass-energy absorption coefficients (calculated with EGSnrc user-code g) of various tissues normalized to those for water water for relevant photon energies ( ${ }^{125}$ I brachytherapy seeds) in keV. Dashed vertical line represents the average energy of ${ }^{125}$ | radionuclide, $28 \mathrm{keV}$. 


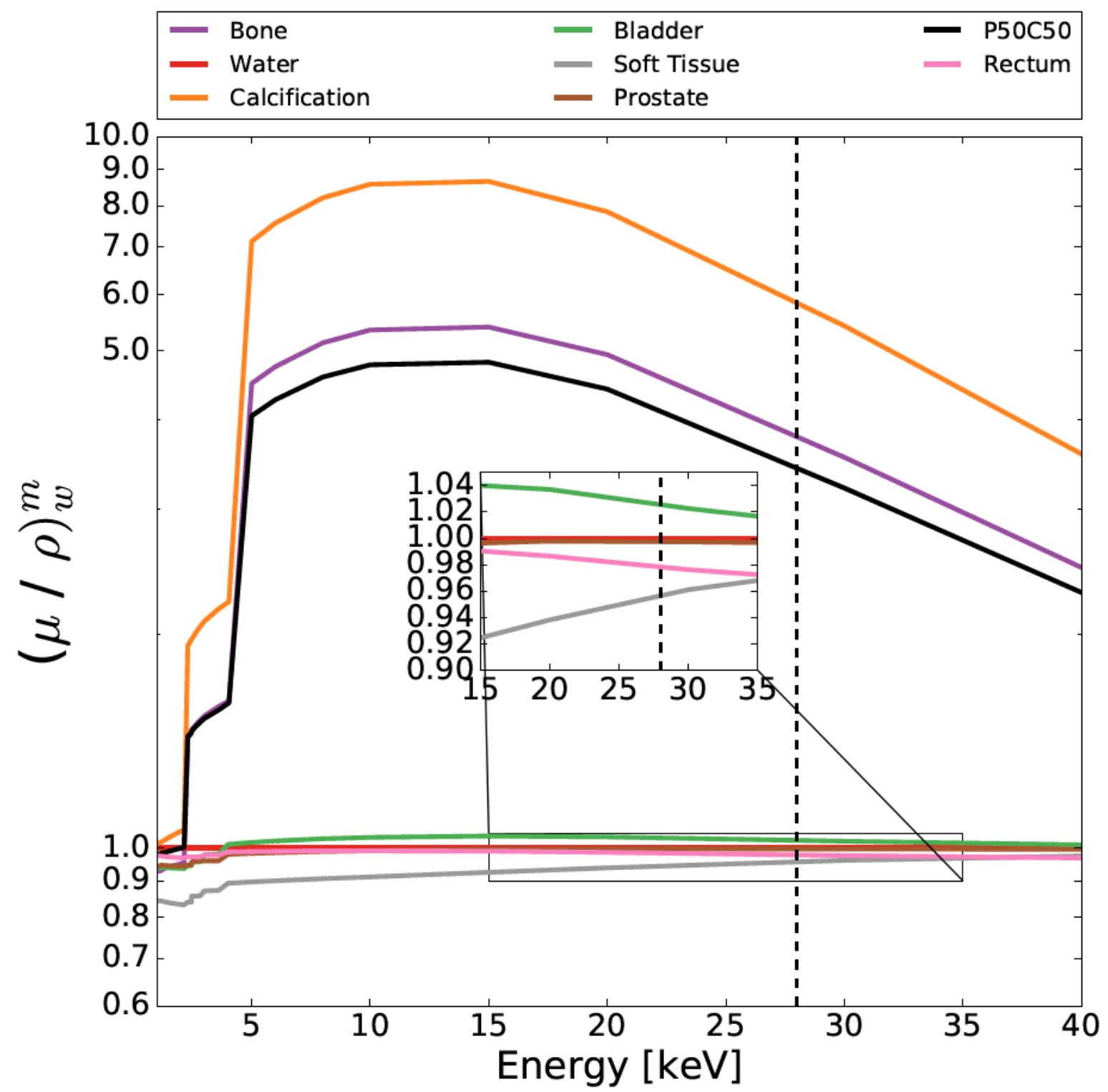

Figure 2.4: Mass attenuation coefficients (from NIST XCOM database ${ }^{51}$ ) of various tissues normalized to those for water for relevant photon energies ( ${ }^{125}$ I brachytherapy seeds) in keV. Dashed vertical line represents the average energy of ${ }^{125} \mid$ radionuclide, $28 \mathrm{keV}$. 
Table 2.2: Elemental composition and mass density of tissues used in tissue assignment scheme. Mass density thresholds are given for tissue assignments applied to various contour-defined regions. CT numbers that correspond to mass densities below $0.0012 \mathrm{~g} / \mathrm{cm}^{3}$ (air) are assigned to stated nominal density.

\begin{tabular}{|c|c|c|c|c|c|c|c|c|}
\hline \multirow{2}{*}{$\begin{array}{l}\text { Image } \\
\text { Region }\end{array}$} & \multirow{2}{*}{$\begin{array}{c}\text { Threshold } \\
{\left[\mathrm{g} / \mathrm{cm}^{3}\right]}\end{array}$} & \multirow{2}{*}{$\begin{array}{c}\text { Tissue } \\
\text { Assignment }\end{array}$} & \multicolumn{5}{|c|}{ Elemental Composition (Mass \%) } & \multirow{2}{*}{$\begin{array}{c}\rho \\
\rho \\
{\left[\mathrm{g} / \mathrm{cm}^{3}\right]}\end{array}$} \\
\hline & & & $\mathrm{H}$ & $\mathrm{C}$ & $\mathrm{N}$ & $\mathrm{O}$ & Elements with $\mathrm{Z}>8$ & \\
\hline \multirow[t]{3}{*}{$\overline{\text { Prostate }}$} & $\overline{[-0.16,1.14]}$ & Prostate ${ }^{20}(\mathbf{P})$ & $\overline{10.5}$ & 8.9 & 2.5 & 77.4 & $\mathrm{Na}(0.2), \mathrm{P}(0.1), \mathrm{S}(0.2), \mathrm{K}(0.2)$ & 1.04 \\
\hline & {$[1.27,8.65]$} & Calcification $^{22}$ (C) & 0.3 & 1.6 & 0.5 & 40.7 & $\mathrm{P}(18.7), \mathrm{Ca}(38.2)$ & 3.06 \\
\hline & {$[1.14,1.27]$} & $50 \%$ P-50\%C Mix & 5.4 & 5.3 & 1.5 & 59.1 & $\mathrm{P}(9.4), \mathrm{S}(0.1), \mathrm{K}(0.1)$ & 1. \\
\hline Blac & {$[-0.1$} & y Bladder (Empty) ${ }^{20}$ & 10.5 & 9.6 & 2.7 & 76.1 & $\mathrm{P}(0.2), \mathrm{S}$ & \\
\hline Rectum & {$[-0.16,8.65]$} & Rectum 21 & 6.3 & 12.1 & 2.2 & 79.0 & $\mathrm{Na}(0.01), \mathrm{P}(0.1), \mathrm{Cl}(0.1), \mathrm{K}($ & 0.75 \\
\hline Urethra & {$[-0.16$} & Pro & 10.5 & 8.9 & 2.5 & 77.4 & $\mathrm{Na}(0.2), \mathrm{P}(0.1), \mathrm{S}(0.2), \mathrm{K}(0.2)$ & 1.04 \\
\hline \multirow[t]{2}{*}{ Other } & {$[-0.16,1.14]$} & Average Male So & 10.5 & 25.6 & 2.7 & 60.2 & $\mathrm{Na}(0.1), \mathrm{P}(0.2), \mathrm{S}(0.3), \mathrm{Cl}(0.2), \mathrm{K}(0.2)$ & 1.03 \\
\hline & {$[1.14,8.65]$} & Corticol Bone ${ }^{20}$ & 3.4 & 15.5 & 4.2 & 43.5 & $\mathrm{Na}(0.1), \mathrm{Mg}(0.2), \mathrm{P}(10.3), \mathrm{S}(0.3), \mathrm{Ca}(22.5)$ & 1.92 \\
\hline
\end{tabular}

\subsubsection{Tissue Assignment and Bladder Contrast Agent}

Some patients were injected with $120 \mathrm{~cm}^{3}$ of $\mathrm{Nal}$ contrast solution via urinary catheter to enhance bladder visibility for delineation. Bladder contrast increases X-ray photon attenuation for the CT scanner that results in an increased CT number (much greater than tissue and similar to bone/calcification) and subsequently increased mass density. This presents two problems when applying a tissue assignment scheme as described above: first, any contrast present outside the bladder contour will be assigned as bone, an anatomically unrealistic scenario; second, the assignment of mass density based on CT number would lead to artificially large mass densities.

Patients with bladder contrast are flagged and corrected before the application of the tissue assignment scheme for virtual patient phantom derivations. For these patients, bladder contours on each slice are enlarged to encompass any contrast leakage outside the physician-defined contours. All voxels within this larger contoured area with CT number greater than $100 \mathrm{HU}$ (chosen to be greater than water or soft tissue) are replaced with $0 \mathrm{HU}$ (this results in mass density equal to unity). Contours are then reset to their original size and the tissue assignment scheme is applied. 
Table 2.3: Statistical uncertainties for each structure averaged over the patient cohort $(\mu)$, one-sigma standard deviations $(\sigma)$, and range of uncertainties, for the two simulation modes. Percentage of voxels included in the statistical uncertainty calculation $^{6,52}$ is also presented (refer to §2.4).

\begin{tabular}{|c|c|c|c|c|c|c|c|c|}
\hline \multirow[b]{2}{*}{ Organ } & \multicolumn{4}{|c|}{$\overline{\text { TG43 }}$} & \multicolumn{4}{|c|}{$M C$} \\
\hline & $\begin{array}{c}\mu \\
{[\%]}\end{array}$ & $\begin{array}{c}\sigma \\
{[\%]}\end{array}$ & $\begin{array}{c}\text { Range [\%] } \\
\text { (Min. - Max.) }\end{array}$ & $\begin{array}{l}\text { Voxels [\%] } \\
\text { Included }\end{array}$ & $\begin{array}{c}\mu \\
{[\%]}\end{array}$ & $\begin{array}{c}\sigma \\
{[\%]}\end{array}$ & $\begin{array}{c}\text { Range [\%] } \\
\text { (Min. - Max.) }\end{array}$ & $\begin{array}{c}\text { Voxels [\%] } \\
\text { Included }\end{array}$ \\
\hline$\overline{\text { Prostate }}$ & $\overline{0.48}$ & $\overline{0.05}$ & 0.26 & 99. & $\overline{0.48}$ & $\overline{0.05}$ & $0.26-0.59$ & 99.8 \\
\hline Bladder & 1.00 & 0.10 & 0.38 & 1 & 1.00 & 0.10 & $0.39-1.20$ & 15 \\
\hline Rectum & 0.80 & 0.08 & 0.56 & 72 & 0.81 & 0.08 & $0.56-0.99$ & 72. \\
\hline Urethra & 0.61 & 0.09 & $0.45-0.77$ & 94.6 & 0.61 & 0.09 & $0.45-0.77$ & 94.6 \\
\hline
\end{tabular}

\subsection{Dose Calculations with BrachyDose}

Virtual patient phantoms had average voxel dimensions of $0.03125 \mathrm{~cm} \times 0.03125 \mathrm{~cm}$ $\times 0.2 \mathrm{~cm}(496 \times 496 \times 56$ voxels $)$. Simulations are run with $10^{9}$ histories to achieve the average statistical uncertainties listed in Table 2.3. For each patient, simulations with heterogeneous tissue assignment with inter-seed effects turned on $(M C)$ are compared to simulations with all voxels in the virtual patient phantom assigned to water (density of $0.998 \mathrm{~g} / \mathrm{cm}^{3}$ ) with inter-seed effects turned off (TG43). Pre-modeled ${ }^{125}$ | brachytherapy sources (Amersham, OncoSeed, 6711) ${ }^{14}$ are superimposed on the virtual patient phantoms at dosimetrist-defined seed centers for both simulations modes: $M C$, and TG43. The ${ }^{125}$ I source spectrum from TG-43U $1^{4}$ is used, with all particles initialized within the source. Radiation transport and dose deposition are performed in medium $\left(D_{m, m}\right)$ for this study. Photon cross-sections are taken from the National Institute of Standards and Technology (NIST) XCOM database ${ }^{51}$. Source spectrum Bound Compton scattering, Rayleigh scattering, atomic relaxations, spin effects, photoelectron angular sampling, electron impact ionization are all modeled. Photon cut-off energy of $1 \mathrm{keV}$ is used, while electron transport is not simulated (electrons are assumed to deposit dose locally, see §1.2). 


\subsection{Dosimetric Analysis}

BrachyDose simulations output a data file (.3ddose) containing dose per simulated history and relative statistical uncertainty for each voxel in the virtual patient model. Dose per history is converted to absolute dose using a dose scaling factor (DSF), given by:

$$
D S F=\frac{S_{k, \text { seed }} \cdot \tau}{S_{k, \text { history }}}
$$

where: $S_{k, \text { seed }}$ is the air kerma strength of the seed (cGy $\mathrm{cm}^{2} \mathrm{~h}^{-1}$ ), and provided in the patient DICOM-RT file; $S_{k, h i s t o r y}$ is the air kerma per history (for Amersham, OncoSeed (6711) $3.7717 \cdot 10^{-14} \mathrm{cGy} \mathrm{cm}^{2} \mathrm{~h}^{-1}$ ) that can be computed using Monte Carlo methods; $\tau$ is the mean lifetime of the radioisotope (for ${ }^{125} \mid 2056.71 \mathrm{hrs}$ ). Using an in-house code to extract dosimetric quantities with the guidance of the physician defined organ contours, cumulative dose-volume histograms (DVH) are derived by binning absorbed dose to fractional organ volume receiving a minimum of said dose. Dose-volume histograms are derived for all contoured organ volumes for both TG43 and $M C$ simulations; dose and volume metrics for each organ are extracted through linear interpolation of the respective DVH.

Dose metrics, denoted $D_{x}$, represent the minimum dose received by $x$ percent of the volume for the relevant structure. In some cases an absolute volume may be used in place of a percentage volume, quoted as $D_{x c c}$, where $x$ is the absolute volume of the organ in cubic centimeters $\left(\mathrm{cm}^{3}\right.$ or $\mathrm{cc}$ ). Volume metrics, denoted $V_{x}$, represent the fractional organ volume that receives at least $x$ percent of the prescription dose $D_{R x}$ (145 Gy for ${ }^{125}$ I brachytherapy). Dose-volume histograms allow absolute dose or fractional organ volume to be expressed quantitatively but lack spatial information, so the use of more qualitative methods is employed to supplement any dose-volume analysis. To visualize spatial dose information, dose distributions are presented as 
a quantitative color gradient (color-scale) that maps out a two-dimensional relative dose map ( $M C$ calculated doses relative to TG43) for a given slice in the patient phantom. Line profiles are used to extract a one dimensional absorbed dose profile through any dimension of the voxelized .3ddose file, for either MC, TG43, or MC/TG43.

Statistical uncertainties to organ volumes defined by contours are calculated by computing the quadrature sum of the individual voxel uncertainties in the volume that receives a dose $\geq 0.25 \cdot D_{R x}$ (145 Gy). Due to the steep dose gradients in brachytherapy, voxels receiving lower doses will have higher relative uncertainties, negatively impacting the overall statistical uncertainty of the dose calculation ${ }^{6,52}$. Additional uncertainties for dose calculations come from uncertainties in the $\mathrm{XCOM}^{51}$ cross-section database, as well as uncertainties in the elemental tissue compositions taken from the literature. Seeing as these uncertainties are applied over the entire cohort, and that this study is mainly focused on comparing MC and TG43 dose distributions, it is expected that these uncertainties will be carried over systematically.

\subsection{Radiobiological Analysis}

Biologically effective dose (BED) is calculated based on the approach outlined in Section 1.4. A voxel-by-voxel approach is taken, where the BED of each voxel within the target volume is computed using Equation (1.7). The final BED value for the patient is a fractional voxel-volume weighted exponential sum of the individual BED values as shown in Equation (1.10). Similarly, tumor control probability (TCP) is calculated using Equation (1.11).

BED and TCP are computed using two methods: (1) discarding voxel doses below 110 Gy, a threshold based on the assumption that these low-doses are in the periphery of the generously delineated target volume as done in Ling et al. ${ }^{38}$, King 
et al. ${ }^{53}$; (2) discarding voxel doses below a low-dose minimum (or dose threshold, $D_{t}$ ), a physical (and mathematical) threshold for a positive $T_{\text {eff }}$.

While the 110 Gy dose cut-off assumption may hold true in the TG-43 paradigm, when using advanced MBDCA and considering inter-seed attenuation and large prostatic calcification, low-dose regions may be present within the tumor volume, an issue discussed in Section 4.2. To employ a dose cut-off to include these low-dose regions, one must take into account the mathematical consequences of low-dose inclusions in the BED and TCP models used. Each individual voxel $B E D_{i}$ must be calculated using Equation (1.7) which depends on an effective treatment time $T_{\text {eff }}$ given by Equation (1.8), repeated below:

$$
T_{\text {eff }}=1.44 T_{1 / 2} \ln \left[\alpha \cdot D \cdot \frac{T_{\text {pot }}}{T_{1 / 2}}\right]
$$

For this equation to make physical sense (and consequently to be mathematically solvable), $T_{\text {eff }}$ must have a positive value:

$$
T_{\text {eff }}=1.44 T_{1 / 2} \ln \left[\alpha \cdot D \cdot \frac{T_{p o t}}{T_{1 / 2}}\right]>0
$$

This restricts the domain of the natural logarithm to being greater than or equal to unity:

$$
\alpha \cdot D \cdot \frac{T_{p o t}}{T_{1 / 2}}>1
$$

$T_{p o t}, T_{1 / 2}$, and $\alpha$ are model parameters and physical constants for the BED equation and therefore constant in this case resulting in a restriction on the dose per voxel, $D$ :

$$
D>\frac{T_{1 / 2}}{T_{p o t} \cdot \alpha}
$$

Using the model parameters listed in Table 2.4 and the half-life of ${ }^{125}$ radionuclide 
(59.43 days), this dose threshold $D_{t}$ becomes:

$$
D_{t}>\frac{59.43 \text { days }}{42 \text { days } \cdot 0.15 \mathrm{~Gy}^{-1}}=9.43 \mathrm{~Gy}
$$

Literature values for $\alpha$ range from $0.019 \mathrm{~Gy}^{-1}-0.35 \mathrm{~Gy}^{-154,55}$, so values for $D_{t}$ range from 4.0 Gy to 74 Gy. The choice of $\alpha\left(=0.15 \mathrm{~Gy}^{-1}\right)$ for this study results in the 9.43 Gy cut-off calculated above.

Modeling parameters used are summarized in Table 2.4 and are consistent with the recommendations of the AAPM TG- $137^{54}$, which referenced works by Wang et al. ${ }^{56,57,58}$.

Table 2.4: Summary of radiobiological parameters used for the calculation of BED for the prostate of each patient.

\begin{tabular}{|c|c|c|}
\hline Parameter & Value & Description \\
\hline$\alpha$ & $0.15 \mathrm{~Gy}^{-1}$ & Intrinsic radiosensitivity of the cells \\
\hline$\alpha / \beta$ & 3.0 Gy & Description of tissue response to dose fractionation \\
\hline $\mathrm{T}_{p o t}$ & 42 days & Potential doubling time of the tumor cell \\
\hline $\mathrm{T}_{r}$ & $0.27 \mathrm{~h}$ & Repair half-life \\
\hline $\mathrm{N}_{0}$ & $10^{6}$ & Total number of tumour cells prior to start of treatment \\
\hline
\end{tabular}

\subsection{Outcomes Study}

Treatment toxicities were scored retrospectively by reviewing each patient's medical record and scored according to The National Cancer Institute (NCl) Common Terminology Criteria for Adverse Events v4.03 (CTCAE) ${ }^{59}$. Relevant radiation toxicities were grouped and recorded under three categories: erectile, urinary, and rectal. For each patient's respective toxicity, the worst observed toxicity (i.e. highest grade) was used. Advanced MBDCA provides accurate representations of patient anatomies compared to the TG-43 approach by modeling heterogeneities in tissue composition and mass density in addition to simulating realistic inter-seed effects. Radiation toxicities are a direct consequence of the brachytherapy treatment, and radiation ex- 
posure of the prostate, OAR, and surrounding healthy tissue. It is hypothesized that MBDCA provides an improved framework for quantifying absorbed dose to tissue that may result in a better prediction of delivered dose and post-treatment outcomes. This study seeks to better correlate differences in absorbed dose to the prostate and OAR calculated with $M C$ compared to TG43. 


\section{Chapter 3}

\section{Results}

\subsection{Dosimetry}

Tissue heterogeneities and inter-seed effects play significant roles in the perturbation of dose distributions calculated according to the TG-43 formalism. To explore and understand the physical intricacies of these effects, three example patients will be presented with results subsequently generalized for the entire patient cohort. Table 3.1 provides a summary of dose-volume metrics for prostate, bladder, and rectum, for the three example patients. Table 3.2 presents a summary of prostate, bladder, rectal, and urethral dose-volume metrics over the entire cohort as calculated by TG43 and $M C$.

\section{Example Patient 1}

For the first example patient, Patient 1, discrepancies between $M C$ and TG43 derived dose distributions will be observed by separating the inter-seed effects (Figure 3.1) and effects attributed to tissue heterogenities (Figure 3.2).

Figure 3.1 presents example Patient 1, where $M C$ and $M C_{n o I S A}(M C$ excluding inter-seed effects) derived doses are compared. This comparison allows one to observe the effect of inter-seed attenuation and scattering separated from tissue effects 


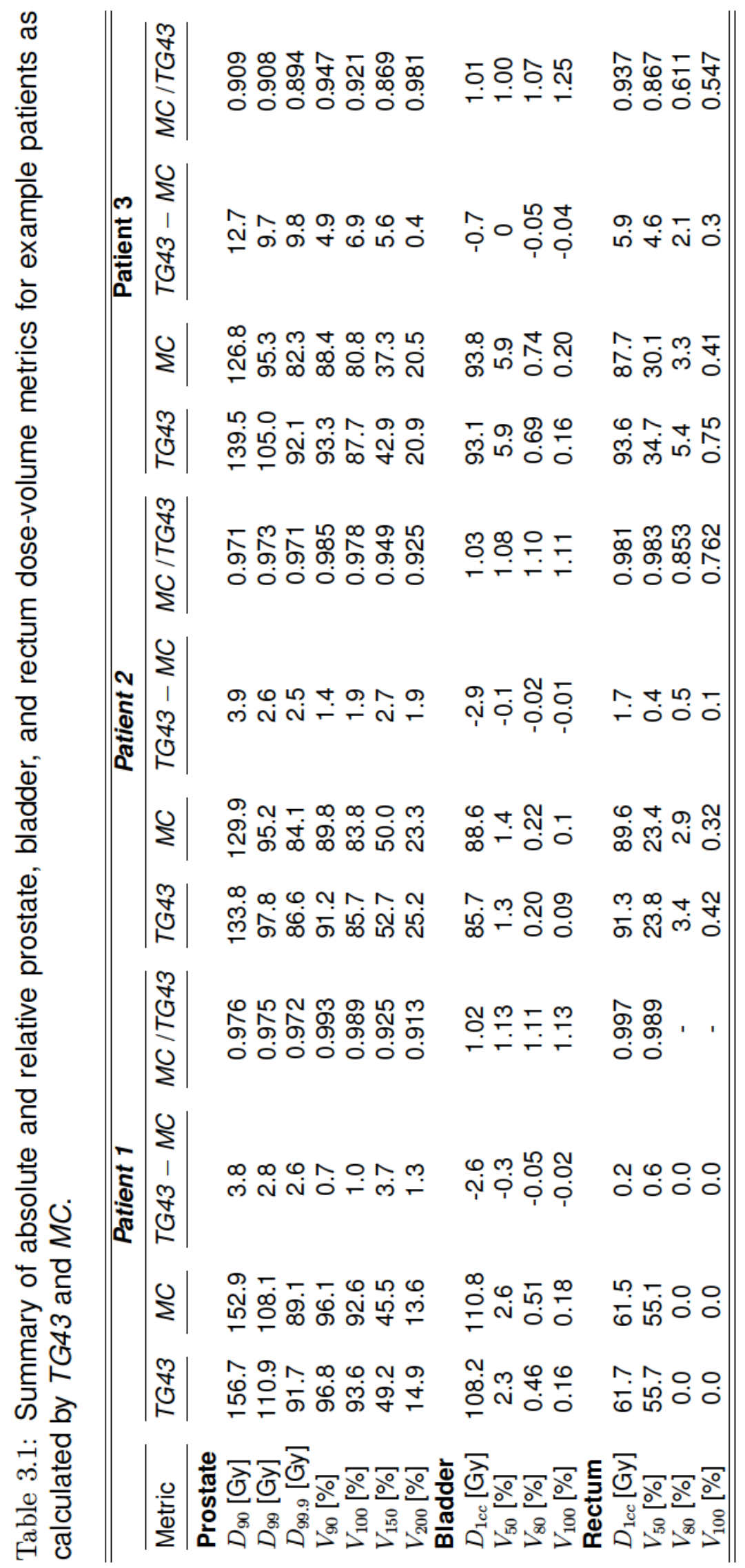



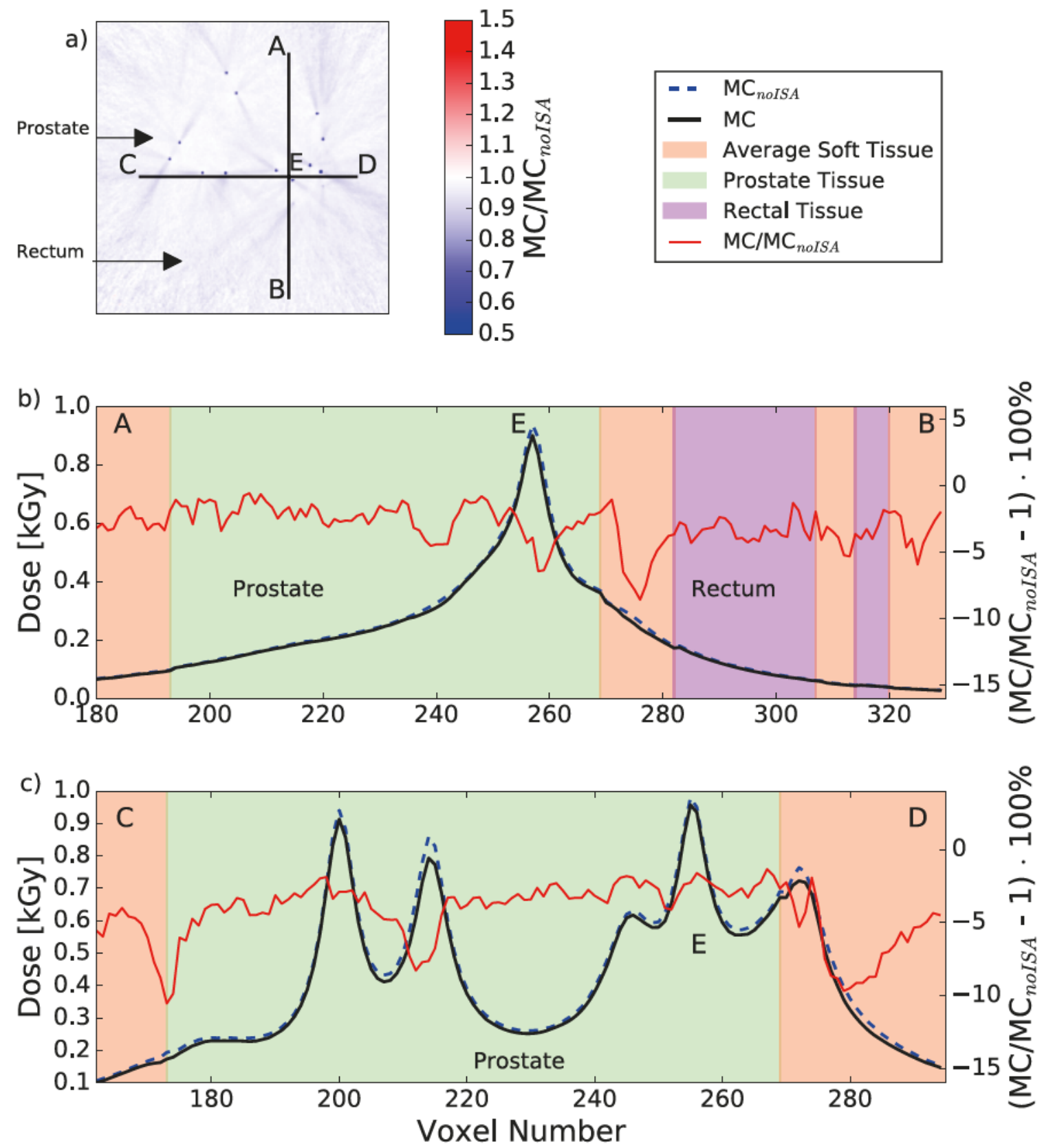

Figure 3.1: Example Patient 1 (a) TG43 normalized dose distribution only considering inter-seed effects with (b) vertical line profile and (c) horizontal line profile. Colorscale indicates MC/TG43 dose ratio for each voxel, with values ranging from $<0.5$ to $>1.5$. Line profiles present dose profiles along the respective direction as calculated by TG43 in Gray (dashed blue line), MC in Gray (solid black line), and MC/TG43 showing percent difference with TG43 (red lines). Voxel tissue assignment indicated with overlaid colors. 
on simulated dose distributions. Prostate, rectal, and bladder dose-volume metrics are all overestimated by $M C_{\text {noISA }}$ : Prostate $D_{90}, V_{100}$, and $V_{200}$ are overestimated by $2.6 \%, 1.8 \%$, and $7.3 \%$, respectively; Rectal $D_{1 c c}$ and $V_{50}$ are overestimated by $3.1 \%$ and $5.0 \%$, respectively; Bladder $D_{1 c c}$ and $V_{50}$ are overestimated by $2.7 \%$ and $8.3 \%$, respectively.

To understand the discrepancies in dose distribution between $T G 43, M C_{n o I S A}$, and $M C$, one must recall Equation (1.2), where under the assumptions of $\mathrm{CPE}$, absorbed dose in a medium is the product of the photon energy $(E)$, photon fluence $(\phi)$, and mass-energy absorption coefficient $\left(\mu_{e n} / \rho\right)$ of the medium. At the low photon energies in ${ }^{125} \mathrm{I}$ brachytherapy ( $\leqslant 35 \mathrm{keV}, \bar{E}_{\gamma}=28 \mathrm{keV}$ ), photoelectric absorption is a significant interaction between the photons and various media (refer to Figure C.1). Photoelectric absorption involves an incident photon interacting with a bound electron in an atom, resulting in the complete absorption of the photon and the emission of a photoelectron (that deposits dose locally). The emitted photoelectron leaves a vacancy in a shell of the atom that may be filled by an electron from a higher shell resulting in fluorescence. Differences in absorbed dose derived using TG43 and $M C$ are generally a result of the differences in photon fluence $(\phi)$ and mass-energy absorption coefficient $\left(\mu_{e n} / \rho\right)$ of the different simulated media.

Figure 3.1(a) presents the $M C_{n o I S A}$ normalized $M C$ dose distribution where lowdose ratio streaks around seed centers attributable to inter-seed attenuation are shown. Photons produced by seeds interact with the titanium capsule ${ }^{32,60,61}$ of neighbouring ${ }^{125}$ s seeds, leading to the photoelectric absorption of the photons and resulting in the attenuation of photon fluence. Since absorbed dose is proportional to photon fluence, a reduction in photon fluence in a medium results in a reduction of absorbed dose. Figure $3.1(\mathrm{~b}, \mathrm{c})$ presents a line profile that traverses seeds in proximity; reductions of up to $10 \%$ in $M C$ dose compared to $M C_{n o I S A}$ are observed 
about seed centers due to the attenuation of photons by the seeds not accounted for in $M C_{\text {noISA. }}$. This effect is not constrained about the seed but extends beyond, in the opposite direction of the neighboring seed (similar to a shadow cast behind an opaque object), resulting in relative dose reductions in $M C$ compared to $M C_{n o I S A}$ in the surrounding regions. These dose reductions are responsible for increased dose in $M C_{n o I S A}$, and are a component of the overall effect when comparing $M C$ and TG43.

Figure 3.2 presents example Patient 1, where TG43 and $M C_{n o I S A}(M C$ excluding inter-seed effects) derived doses are compared. This comparison allows one to observe the effect of tissue inhomogeneities on simulated dose distributions in the absence of inter-seed effects. Prostate dose-volume metrics are relatively higher, while rectal and bladder dose-volume metrics are relatively lower for $M C_{n o I S A}$ calculations compared to TG43: Prostate $D_{90}, V_{100}$, and $V_{200}$ are lower by $0.39 \%, 0.30 \%$, and $0.37 \%$, respectively; Rectal $D_{1 c c}$ and $V_{50}$ are higher by $1.2 \%$ and $3.2 \%$, respectively; Bladder $D_{1 c c}$ and $V_{50}$ are higher by $6.3 \%$ and $21 \%$, respectively.

Differences between the TG43 and $M C$ derived dose distributions are a result of deviations of $\mu_{e n} / \rho$ of the simulated medium compared to water. Since absorbed dose is proportional to the mass-energy absorption coefficient $\left(\mu_{e n} / \rho\right)$ of the medium (which describes the probability of interaction), an increase or decrease of $\mu_{e n} / \rho$ in the medium compared to water will result in an increase or decrease of absorbed dose in the medium compared to water. Similarly, differences in mass absorption coefficient $(\mu / \rho)$ between water and simulated medium result in difference in the attenuation of photon fluence. Since absorbed dose is proportional to photon fluence, any increase or decrease in photon fluence in a medium compared to water leads to an increase or decrease in absorbed dose in the medium compared to water.

Figures 3.2(b, c) presents $T G 43, M C_{n o I S A}$ and $M C_{n o I S A} / T G 43$ doses for two line 

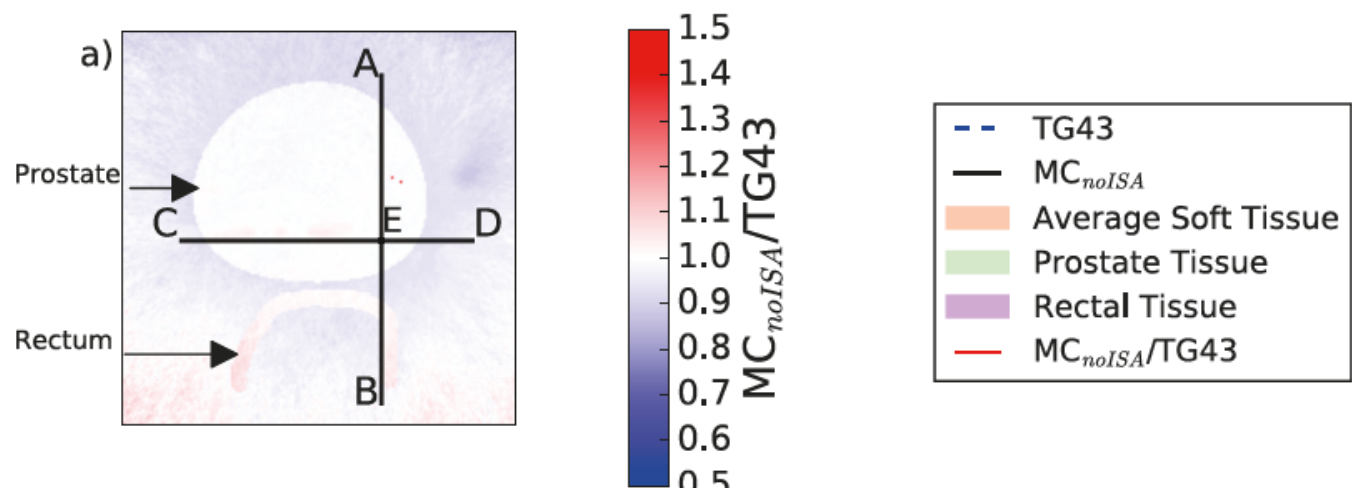

0.5
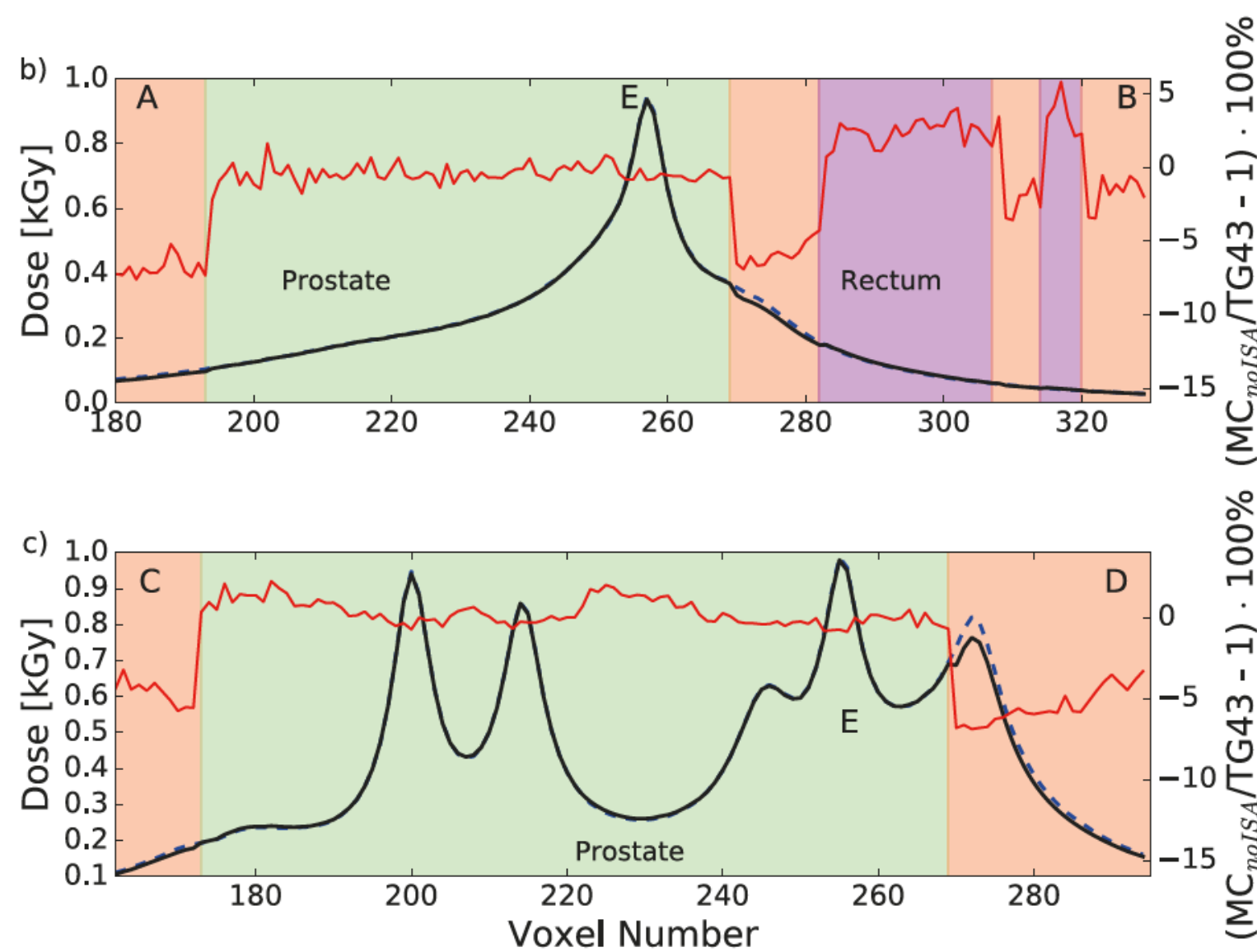

Figure 3.2: Example Patient 1 (a) TG43 normalized dose distribution only considering tissue effects with (b) vertical line profile and (c) horizontal line profile. Color-scale indicates $M C / T G 43$ dose ratio for each voxel, with values ranging from $<0.5$ to $>1.5$. Line profiles present dose profiles along the respective direction as calculated by TG43 in Gray (dashed blue line), MC in Gray (solid black line), and MC/TG43 showing percentage difference with TG43 (red lines). Voxel tissue assignment indicated with overlaid colors. 
profiles that traverse prostate tissue, intermediary soft male tissue, and rectal tissue. In the prostate region, it is observed that there is a small difference between $M C_{n o I S A}$ and TG43; prostate tissue is very similar to water tissue (shown in Figures 2.3 and 2.4) with similar mass attenuation coefficients $\left((\mu / \rho)_{W \text { ater }}^{\text {Prosta }}=0.998\right.$ at 28 $\mathrm{keV})$ and mass energy-absorption coefficients $\left(\left(\mu_{e n} / \rho\right)_{\text {Water }}^{\text {Proste }}=0.993\right.$ at $\left.28 \mathrm{keV}\right)$. Prostate tissue will generally have decreased photon fluence and energy-absorption compared to water resulting in small differences dosimetrically between assigning water versus prostate in the prostate region. Soft male tissue, compared to water, has a lower mass attenuation coefficient $\left((\mu / \rho)_{\text {Water }}^{\text {Softissue }}=0.953\right.$ at $\left.28 \mathrm{keV}\right)$ and mass-energy absorption coefficient $\left(\left(\mu_{\text {en }} / \rho\right)_{\text {Water }}^{\text {SoftTisue }}=0.929\right.$ at $\left.28 \mathrm{keV}\right)$ resulting in decreased photon attenuation and energy-absorption in soft tissue versus water. Figures 3.2(b, c) illustrate dose decreases in $M C_{\text {noISA }}$ compared to TG43 since photons traveling through soft male tissue experience less attenuation and decreased energy-absorption compared to water. This decreased attenuation of photon fluence in the intermediary soft tissue region leads to increased photon fluence incident on the rectum in $M C_{n o I S A}$ compared to TG43. Rectal tissue has a lower mass absorption coefficient $\left((\mu / \rho)_{\text {Water }}^{\text {Rectum }}=0.980\right.$ at $\left.28 \mathrm{keV}\right)$ and mass energy-absorption coefficient compared to water $\left(\left(\mu_{e n} / \rho\right)_{\text {Water }}^{\text {Rectum }}=0.995\right.$ at $\left.28 \mathrm{keV}\right)$. Since the incident photon fluence is increased and rectal tissue attenuates photon fluence less compared to water, rectal dose is generally underestimated by TG43, as shown in Figures3.2(a, b).

Figure 3.3 presents example Patient 1, comparing $M C$ and TG43 dose distributions to show the overall effect of considering tissue heterogeneities and inter-seed effects. The overall effect can be roughly imagined as the "superposition" of the interseed effects in Figure 3.1 and tissue effects in Figure 3.2. Prostate $D_{90}, V_{100}$, and $V_{200}$ are overestimated by TG43 by $2.9 \%, 2.2 \%$, and $7.5 \%$, respectively. Rectal $D_{1 c c}$ and $V_{50}$ are overestimated by TG43 by $1.9 \%$ and $1.7 \%$, respectively. Bladder $D_{1 c c}$ and 

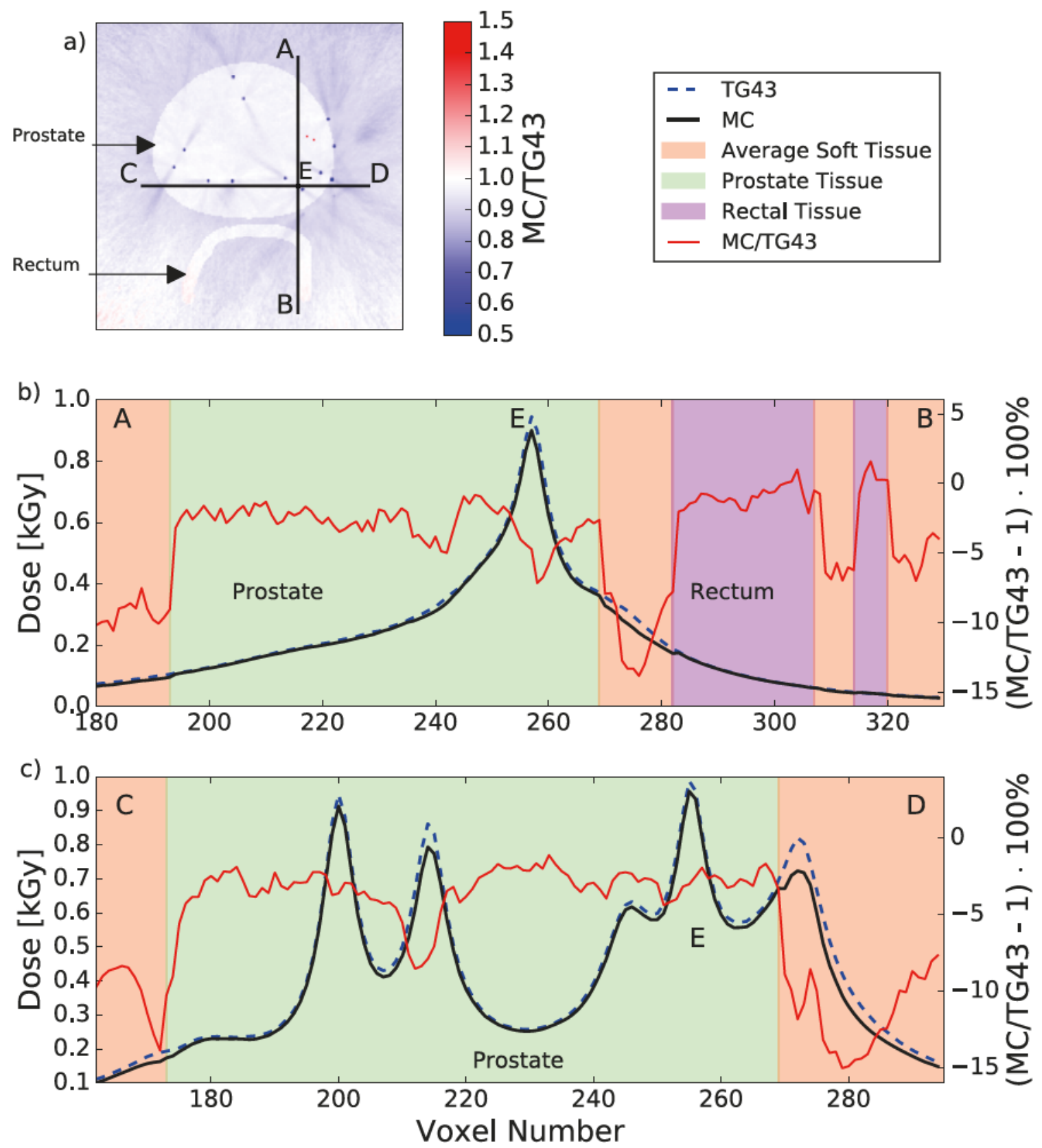

Figure 3.3: Example Patient 1 (a) TG43 normalized dose distribution showing interseed and tissue effects with (b) vertical line profile and (c) horizontal line profile. Color-scale indicates MC/TG43 dose ratio for each voxel, with values ranging from $<0.5$ to $>1.5$. Line profiles present dose profiles along the respective direction as calculated by TG43 in Gray (dashed blue line), MC in Gray (solid black line), and MC/TG43 showing percent difference with TG43 (red lines). Voxel tissue assignment indicated with overlaid colors. 
$V_{50}$ are underestimated by $T G 43$ by $3.0 \%$ and $8.0 \%$, respectively. Inter-seed effects play the most significant role in the overall reduction of $M C$ dose compared to TG43 for this patient.

\section{Example Patient 2}

Figure 3.4 presents the second example patient, Patient 2, where TG43 overestimates prostate and rectal dose-volume metrics, while underestimating bladder dosevolume metrics, compared to $M C$. Prostate $D_{90}, V_{100}$, and $V_{200}$ are overestimated by TG43 by $2.4 \%, 1.1 \%$, and $8.7 \%$, respectively. Rectal $D_{1 c c}$ and $V_{50}$ are overestimated by $0.30 \%$ and $1.1 \%$ by TG43, respectively. TG43 underestimates bladder $D_{1 c c}$ and $V_{50}$ by $2.0 \%$ and $13 \%$, respectively.

Figure $3.4(\mathrm{~b})$ presents a line profile that traverses a vertical segment on the slice of the patient phantom indicated by the vertical line on Figure 3.4(a); doses calculated with both $M C$ and TG43 follow the same trend, however, the MC/TG43 line indicates relative differences of about $-30 \%-5 \%$ between $M C$ and TG43. Similar to Patient 1 , a small decrease in $M C$ dose compared to $T G 43$ is observed in the prostate region (mostly due to inter-seed effects). Bladder has a larger mass attenuation and mass-energy absorption coefficient compared to water (refer to §2.2.3), leading to increased attenuation and energy-absorption for photons simulated in the bladder. Since photon fluence incident on the bladder is increased in $M C$ compared to TG43, due to the intermediary soft tissue that attenuates photons less than water, in addition to a larger mass energy-absorption coefficient of bladder compared to water, dose to bladder voxels adjacent to the prostate region are initially underestimated by TG43. Further into the bladder region, moving away from the prostate, TG43 begins to overestimate dose since bladder attenuates photons more (decreased photon fluence) than water. Overall, bladder dose-volume metrics are underestimated 

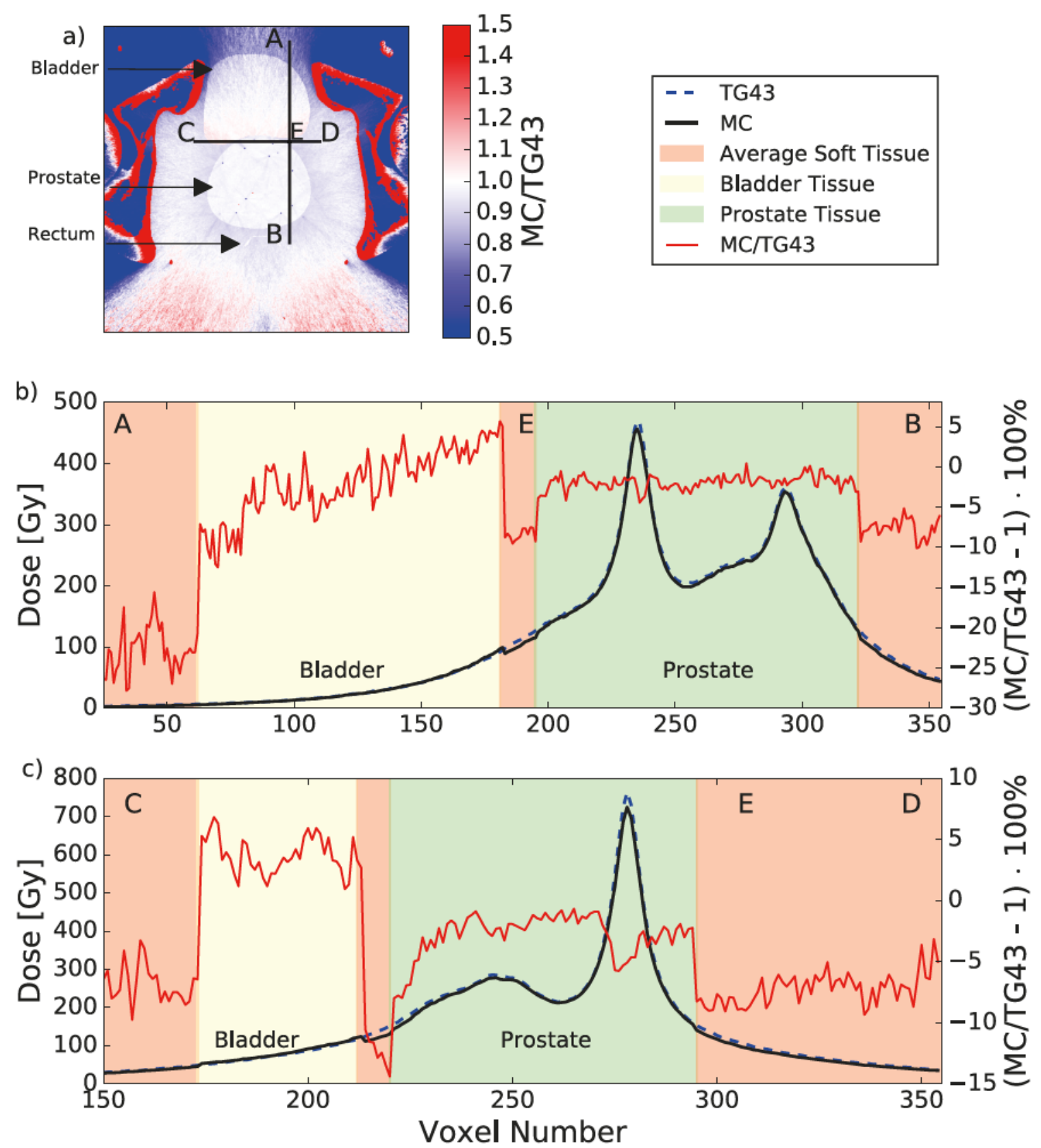

Figure 3.4: Example Patient 2 (a) TG43 normalized dose distribution with (b) vertical line profile and (c) horizontal line profile. Color-scale indicates MC/TG43 dose ratio for each voxel, with values ranging from $<0.5$ to $>1.5$. Line profiles present dose profiles along the respective direction as calculated by TG43 in Gray (dashed blue line), $M C$ in Gray (solid black line), and MC/TG43 showing percent difference with TG43 (red lines). Voxel tissue assignment indicated with overlaid colors. 
by $T G 43$ for this patient.

\section{Patient 3}

Figure 3.5 presents the third example patient, Patient 3 , that had the largest discrepancy of prostate $D_{90}$ between $T G 43$ and $M C$, out of all the patients in the cohort, with TG43 overestimating prostate $D_{90}$ by $9.12 \%$. Furthermore, prostate $V_{100}$ and $V_{200}$ were overestimated by TG43 by $7.9 \%$ (largest discrepancy in cohort) and $1.9 \%$, respectively. Rectal $D_{1 c c}$ and $V_{50}$ are overestimated by $6.3 \%$ (largest in the cohort) and $13.3 \%$, respectively. Bladder $D_{1 c c}$ and $V_{50}$ are underestimated by $1.0 \%$ and $0.87 \%$, respectively.

This large discrepancy in prostate $D_{90}$ and $V_{100}$ are due to the large calcification, which is labeled in Figure 3.5(a) (for a single slice). Calcification tissue, compared to water, has a much larger mass attenuation coefficient $\left((\mu / \rho)_{\text {Water }}^{\text {Calcifion }}=6.3\right)$ and mass energy-absorption coefficient $\left(\left(\mu_{e n} / \rho\right)_{\text {Water }}^{\text {Calcifica }}=11\right)$; calcification tissue will attenuate photons and absorb a considerably larger amount of energy compared to water. While the dose to the calcification tissue is of no clinical significance, dose reductions as a result of these large structures are of interest. Since there is a decrease in photon fluence, there is an accompanying decrease in absorbed dose for the $M C$ scheme compared to $T G 43$ in a large prostate region surrounding the calcification tissue. Figure 3.5 (b) shows a reduction of up to $45 \%$ in absorbed dose between $M C$ and TG43 in the prostate adjacent to the calcification. Similarly, Figure 3.5(c) illustrates calcification tissue absorbing up to $311 \%$ more dose than water, with reductions of up to $45 \%$ in the prostate doses in the surrounding region. 


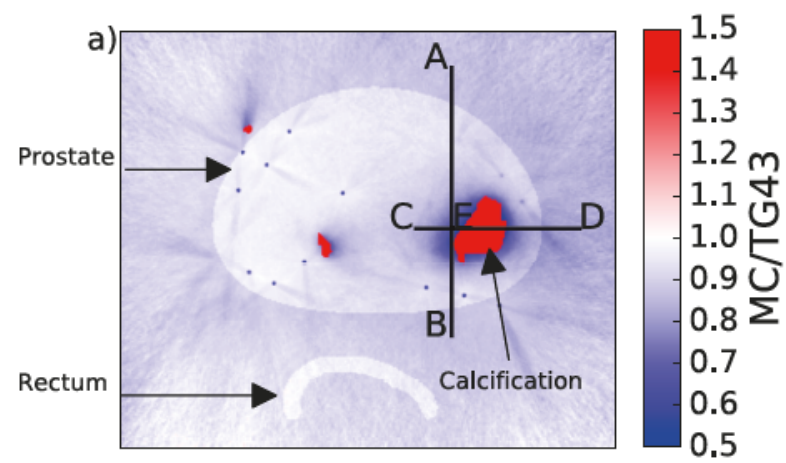

1.5

1.4

1.3

$1.2 \mathrm{~m}$

1.1 过

(1.0

$0.8 \Sigma$

0.7

0.5

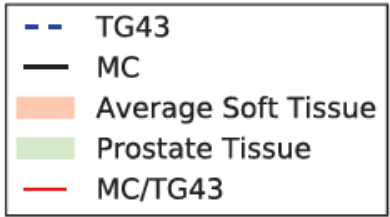

b)

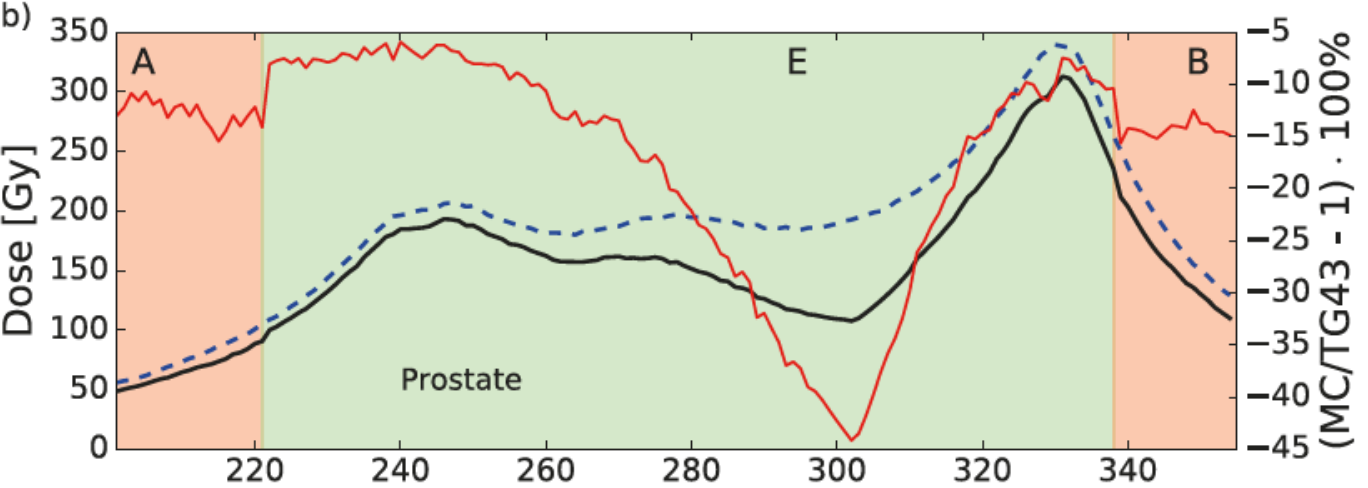

c) 800

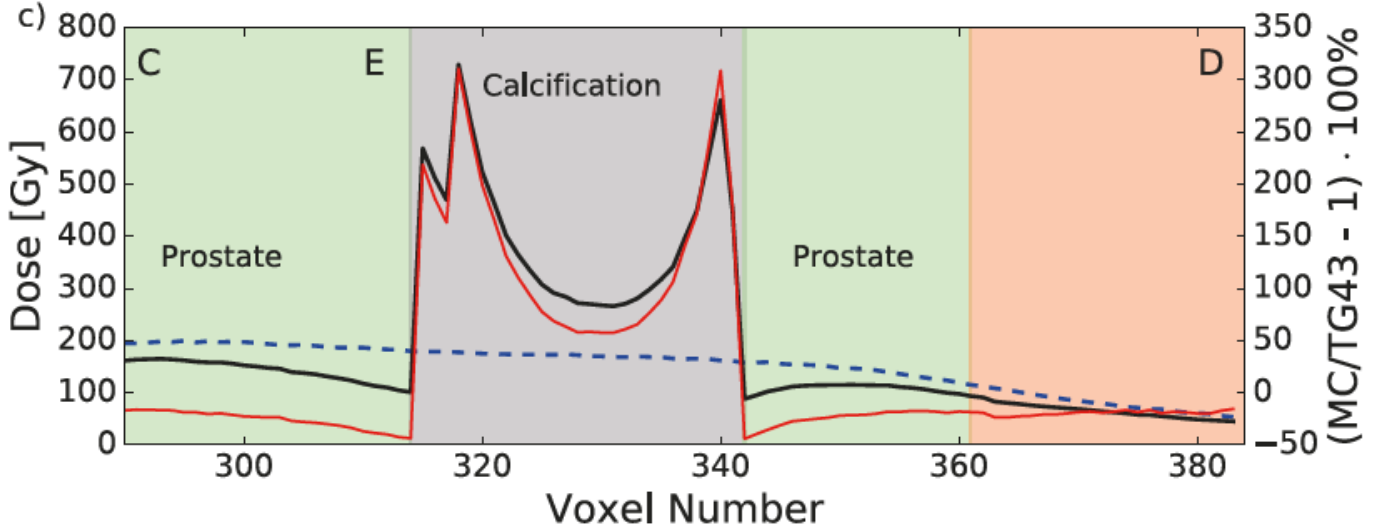

Figure 3.5: Example Patient 3 (a) TG43 normalized dose distribution with (b) vertical line profile and (c) horizontal line profile. Color-scale indicates MC/TG43 dose ratio for each voxel, with values ranging from $<0.5$ to $>1.5$. Line profiles present dose profiles along the respective direction as calculated by TG43 in Gray (dashed blue line), $M C$ in Gray (solid black line), and MC/TG43 showing percentage difference with TG43 (red lines). Voxel tissue assignment indicated with overlaid colors. 


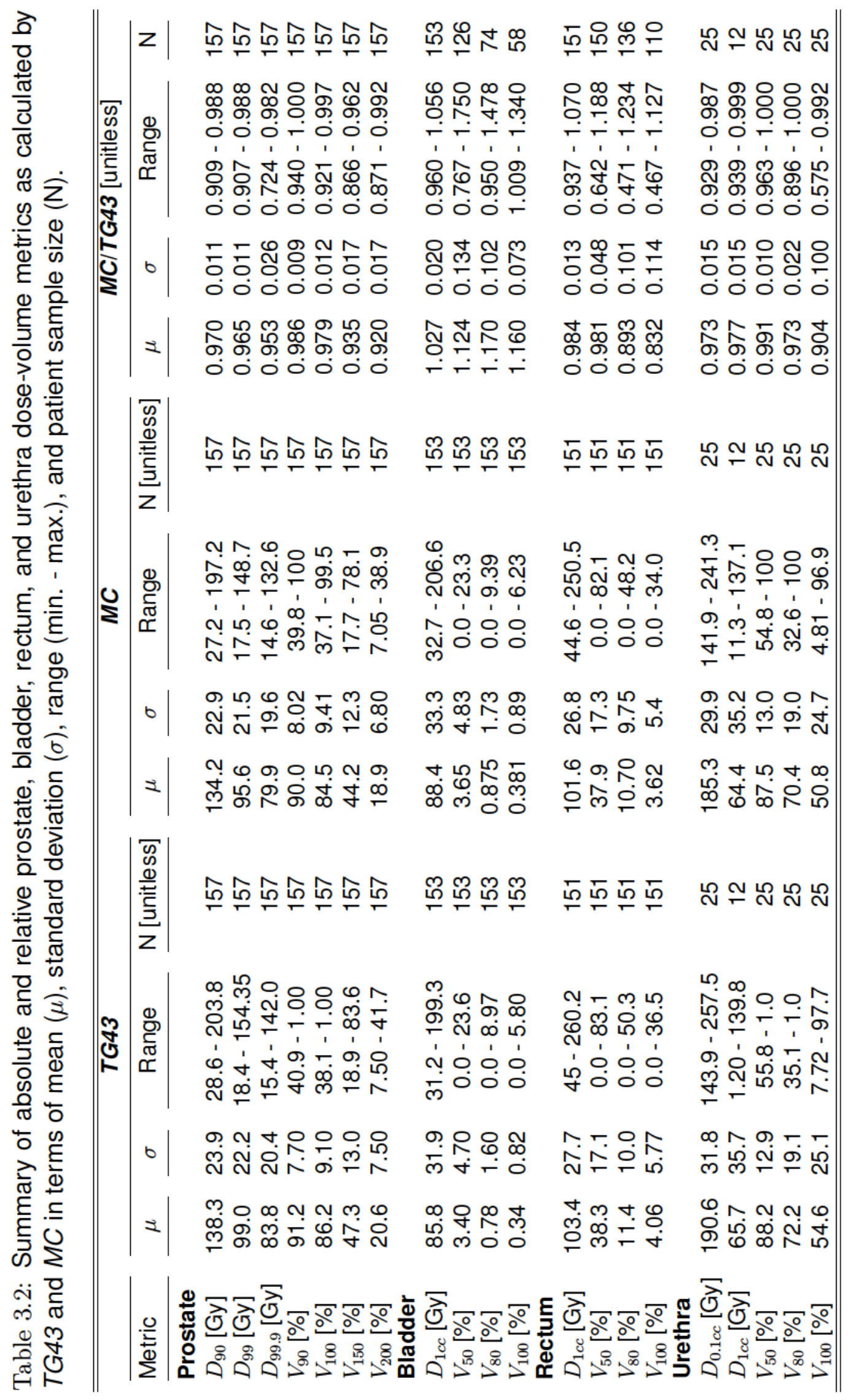




\subsubsection{Prostate}

Figure 3.6 compares the absolute distribution of prostate $D_{90}, D_{99}$, and $D_{99.9}$ as calculated with TG43 and MC for a cohort of 157 patients. As the prostate volume of interest for calculating minimum absorbed dose is increased (minimum dose received by $90 \%, 99 \%$, and $99.9 \%$ of the prostate) there is a decrease in average dose metric. Similarly, when looking at prostate volume metrics, presented in Figure 3.7 for $V_{90}, V_{100}, V_{150}$, and $V_{200}$, as the minimum absorbed dose threshold is increased, the volume receiving said dose decreases.

When comparing dose-volume metrics calculated with the two simulation approaches, prostate dose-volume metrics are overestimated by TG-43 (as simulated with TG43) compared to when tissue heterogeneities and inter-seed effects are taken into consideration $(M C)$. As seen when comparing both panels of Figures 3.6 and 3.7, separately, there is a systematic decrease in average dose-volume metric for $M C$ compared to TG43. To illustrate the overall effect of considering tissue heterogeneities and inter-seed effects on prostate dose-volume metrics, Figure 3.8 presents all the prostate dose-volume metrics calculated with $M C$ normalized to TG43 for each patient. On average, there is an overestimation by TG43 of $2.98 \%$, $3.48 \%$, and $4.7 \%$ for prostate $D_{90}, D_{99}$, and $D_{99.9}$, respectively. Additionaly prostate $V_{90}, V_{100}, V_{150}$, and $V_{200}$, are overestimated by $T G 43$ by $1.37 \%, 2.06 \%, 6.54 \%$, and $8.02 \%$, on average, respectively.

Figure 3.9 presents prostate $\left(D_{90}\right)_{T G 43}^{M C}$ (the ratio of $D_{90}$ calculated by $M C$ normalized to $T G 43)$ and $\left(V_{100}\right)_{T G 43}^{M C}$ as a function of the fraction of the prostate volume assigned to calcification. For prostate $\left(D_{90}\right)_{T G 43}^{M C}$, most patients (134 of the 157 total) had dose ratios within one standard deviation of the mean. Patients with $\left(D_{90}\right)_{T G 43}^{M C}$ 


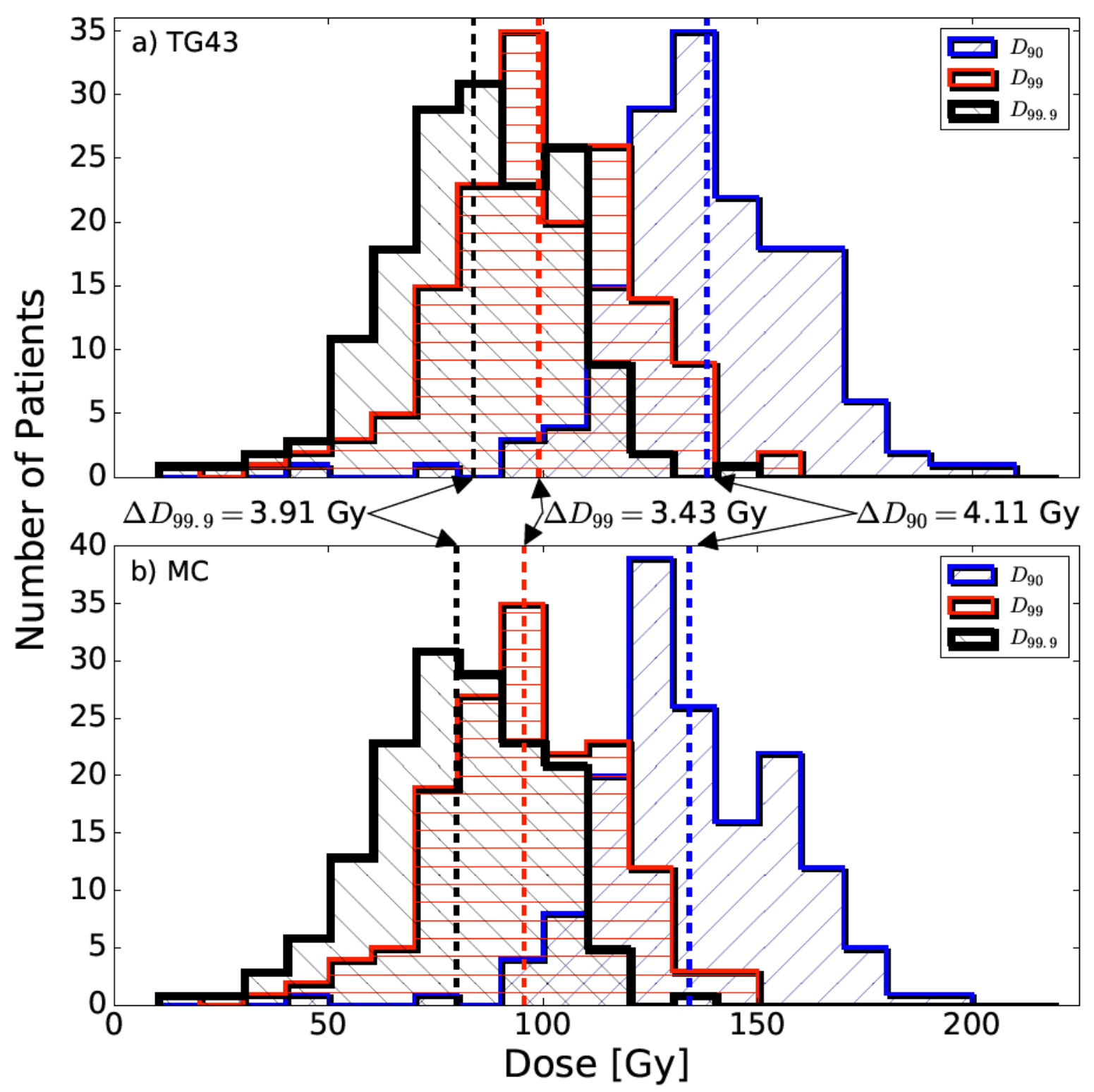

Figure 3.6: Distribution of prostate dose metrics, $D_{90}, D_{99}$, and $D_{99.9}$, for the cohort of patients $(\mathrm{N}=157)$ as calculated with (a) TG43, and (b) MC. Vertical dashed lines represent mean absolute dose value for each corresponding histogram. $\Delta D_{x}$ represents difference between average dose metrics calculated by TG43 and MC. 


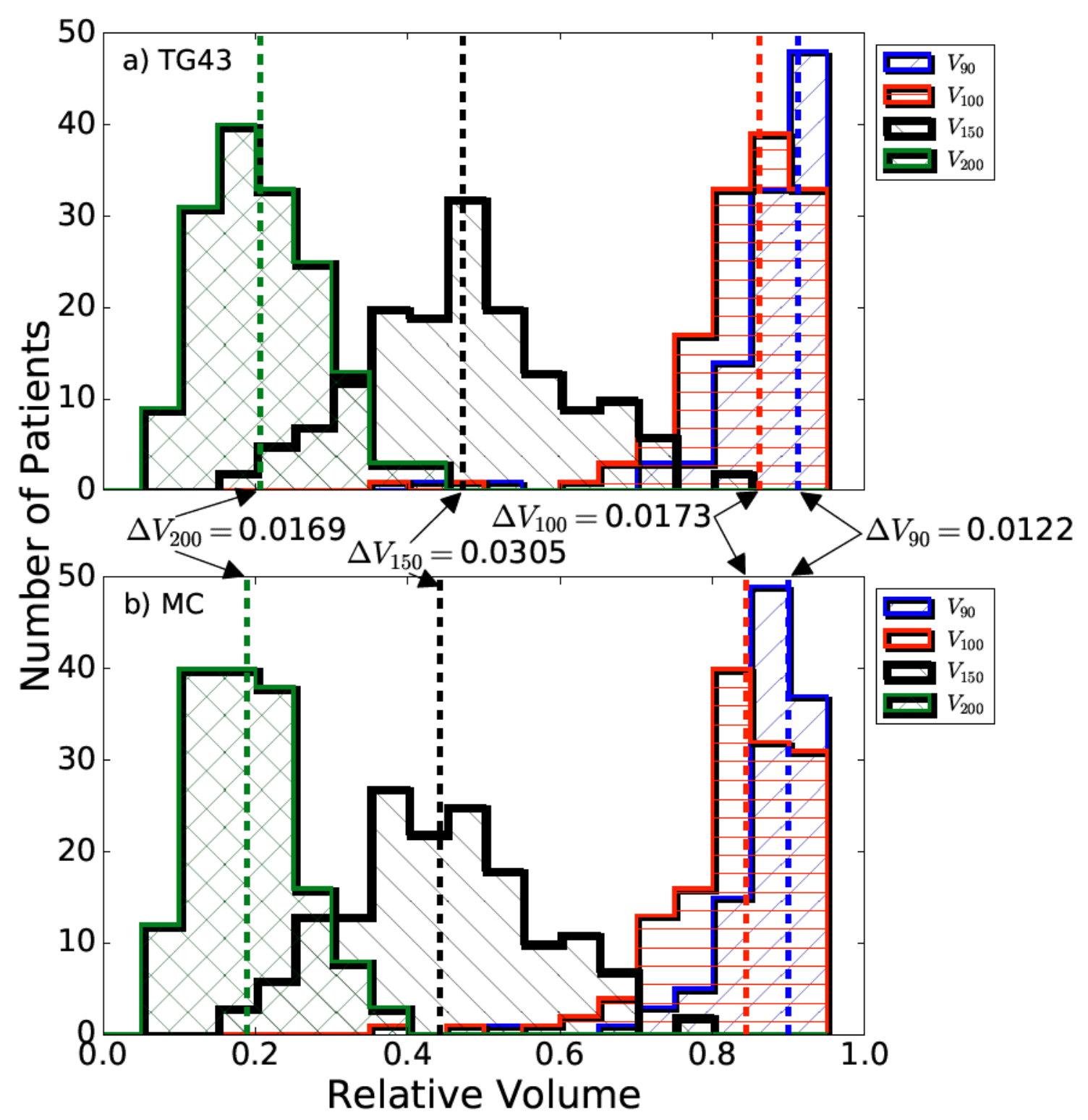

Figure 3.7: Distribution of prostate volume metrics, $V_{90}, V_{100}, V_{150}$, and $V_{200}$, for a cohort of patients $(\mathrm{N}=157)$ as calculated with (a) $T G 43$, and (b) MC. Vertical dashed lines represent mean relative volumes. $\Delta V_{x}$ represents difference between average volume metrics calculated by $T G 43$ and $M C$. 


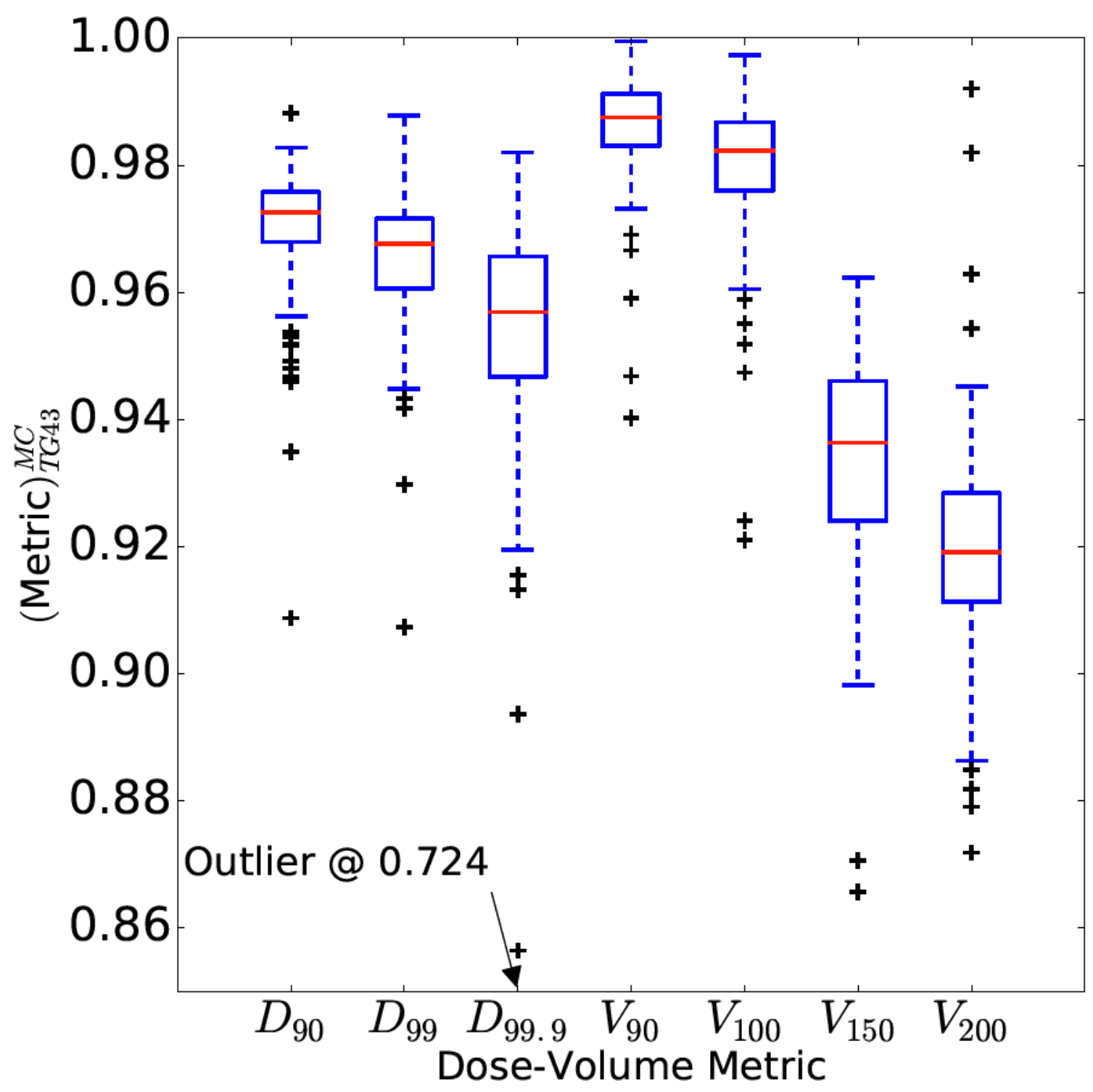

Figure 3.8: Prostate dose-volume metrics calculated with MC normalized to TG43 calculations. The bottom and top of the boxes represent the first (Q1) and third (Q3) quartile (Inter-quartile Range $(I Q R)=Q 3-Q 1)$, respectively; the median is represented by the horizontal band. Top and bottom whiskers extend to (Q3 + 1.5 $\times I Q R)$ and $(\mathrm{Q} 1-1.5 \times I Q R)$, respectively, with outliers marked. 
less than one standard deviation from the mean were generally those with a nonnegligible fraction of their prostate voxels assigned to calcification tissue. A similar trend for $\left(V_{100}\right)_{T G 43}^{M C}$ is observed, in that patients with a large fraction of prostate assigned to calcification showed larger discrepancies between TG43 and MC dose calculations. The patient with the largest deviation of dose-volume metrics from TG43 and the largest percentage of prostate volume assigned calcification was example Patient 3, investigated in §3.1.

In general, it is shown that all prostate dose metrics are overestimated by dose calculations performed in accordance with the TG-43 formalism, i.e. water-based patient phantoms with dose contributions of each seed considered independently. With the consideration of tissue heterogeneities and inter-seed effects in the $M C$ scheme, an average overestimation by $T G 43$ of $2.98 \%$ for prostate $D_{90}$ and $2.06 \%$ for $V_{100}$ are observed. For the most extreme cases, $D_{90}$ overestimation of up to $9.12 \%$ are possible due to the presence of low-dose regions caused by large prostatic calcifications. 


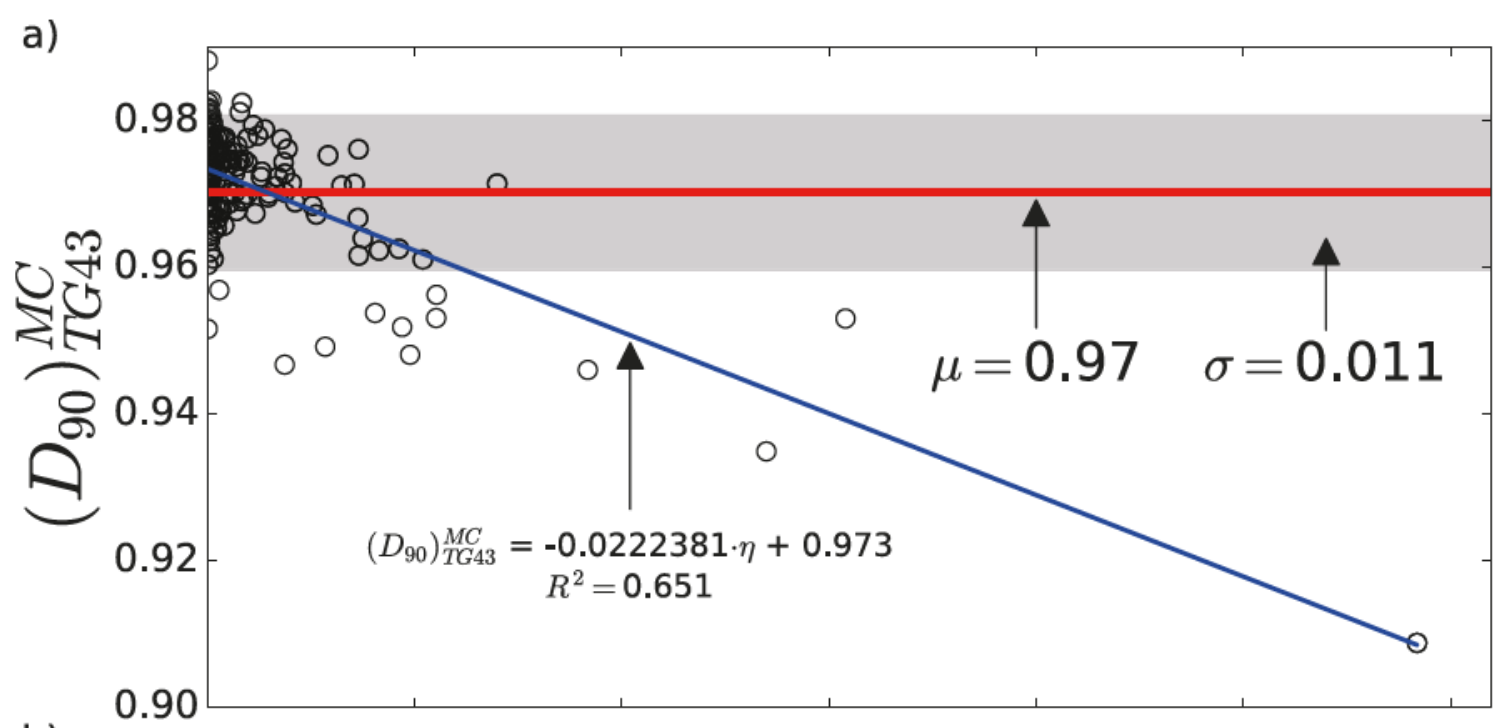

b)

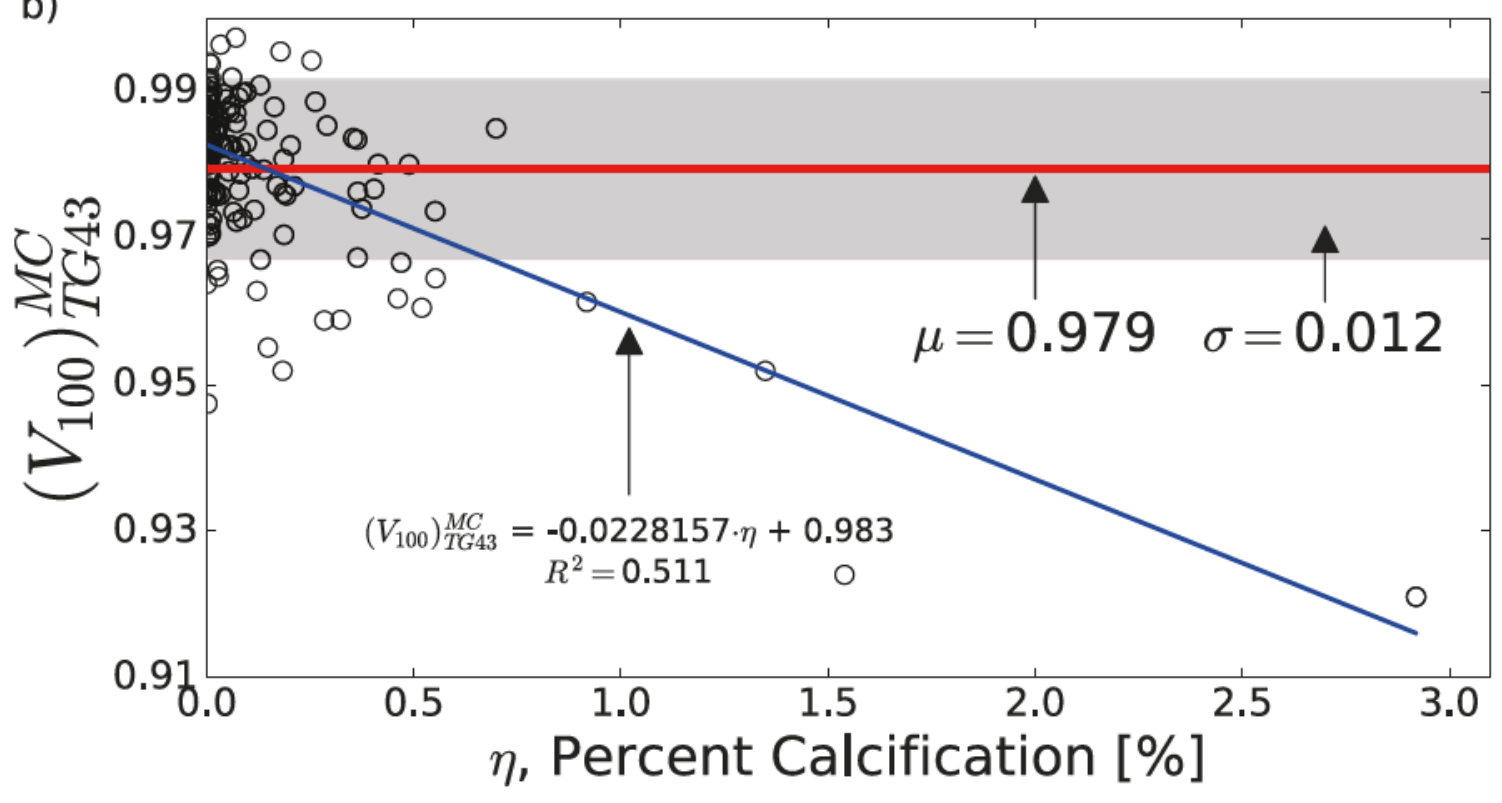

Figure 3.9: Prostate $D_{90}$ and $V_{100}$ metrics calculated with $M C$ normalized to TG43 as a function of percent calcification in the prostate region. For each normalized metric, the mean and one standard deviation is highlighted, in addition to a firstdegree polynomial fit showing the overall relation between percent calcification and the two prostate dose-volume metric ratios. 


\subsubsection{Organs-at-Risk}

Figure 3.10 presents the distribution of absolute dose-volume metrics for all organsat-risk (OAR) (bladder, rectum, and urethra), comparing dose-volume distributions calculated by TG43 and MC. Figure 3.11 presents the same set of dose-volume metrics for the OAR, normalizing $M C$ dose calculations to $T G 43$, removing absolute fractional volumes below $0.1 \%$.

This study has shown that bladder dose-volume metrics are generally underestimated by TG43. Bladder tissue has a larger mass attenuation and mass energyabsorption coefficient compared to water that leads to a comparatively increased photon attenuation, and photon energy-absorption in the bladder region. While these two effects are competing, dose-volume metrics generally consider the region of the bladder adjacent to the prostate, where TG43 underestimates the dose compared to MC. Bladder $D_{1 c c}$ is underestimated by $T G 43$ by $2.78 \%$, on average. Bladder $V_{50}$, $V_{80}$, and $V_{100}$ are also underestimated by $T G 43$ by an average of $12.4 \%, 17.0 \%$, and $16.0 \%$, respectively.

The rectum has a photon mass attenuation and photon mass-energy absorption coefficient lower than water resulting in decreased photon attenuation and photon energy-absorption. For some patient cases, an underestimation by TG43 of rectal dose-volume metrics was observed, but on average, these dose-volume metrics are overestimated by TG43 compared to MC. Rectal $D_{1 c c}$ is overestimated by TG43 by $1.62 \%$, on average, while rectal $V_{50}, V_{80}$, and $V_{100}$, are overestimated by an average of $1.67 \%, 10.7 \%$, and $16.8 \%$, respectively.

While being in the middle of the treatment volume, urethral $D_{1 c c}$ is on average lower than both bladder and rectal $D_{1 c c}$, as shown in Figure 3.10(a). Urethra is as- 

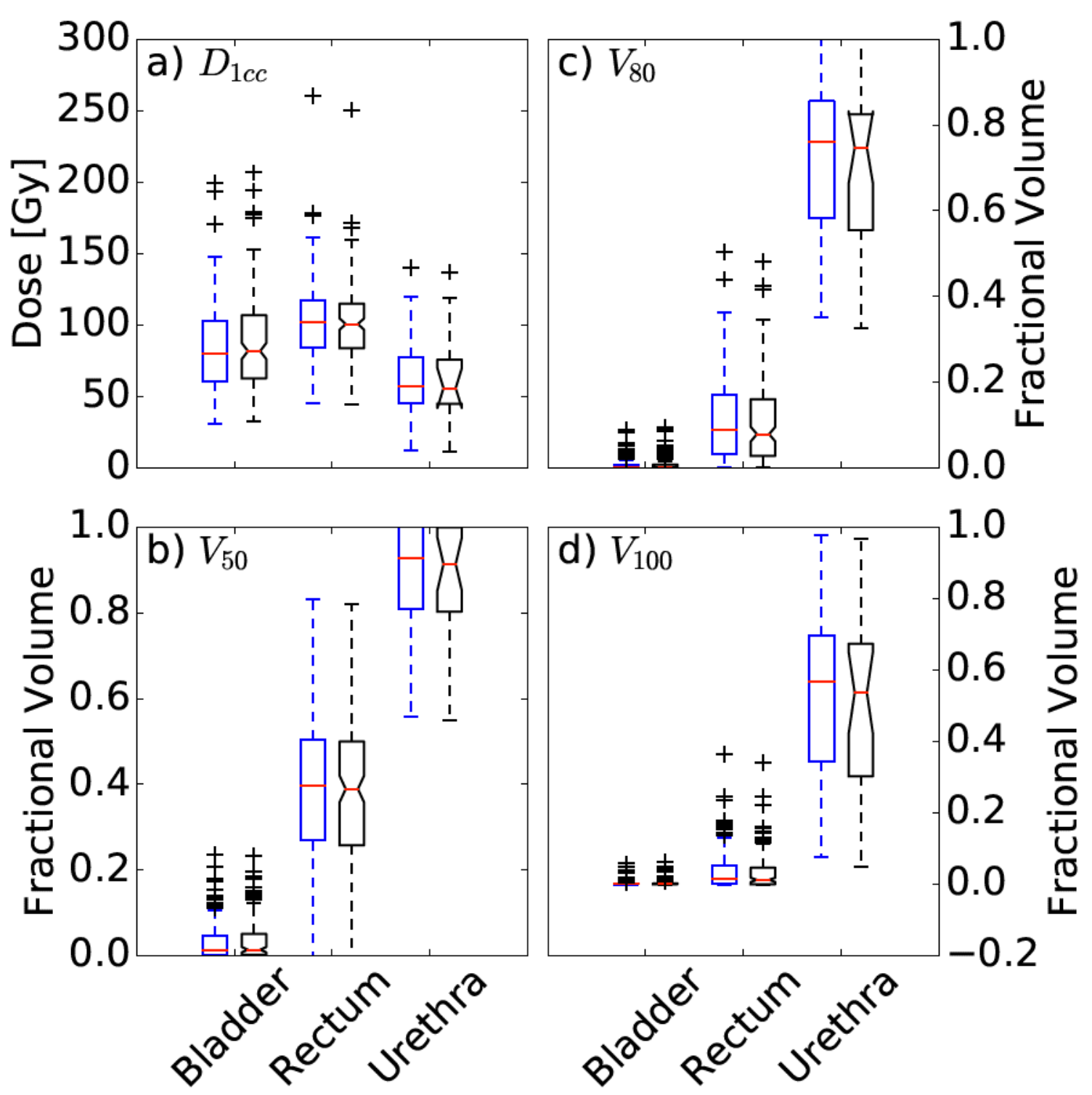

Figure 3.10: Box and whiskers plot of absolute distribution of organs-at-risk dosevolume metrics. Blue boxes represent TG43 calculations while notched black boxes represent $M C$ calculations. $\mathrm{N}_{\text {Bladder }}=154, \mathrm{~N}_{\text {Rectum }}=152, \mathrm{~N}_{\text {Urethra }}=12$. 

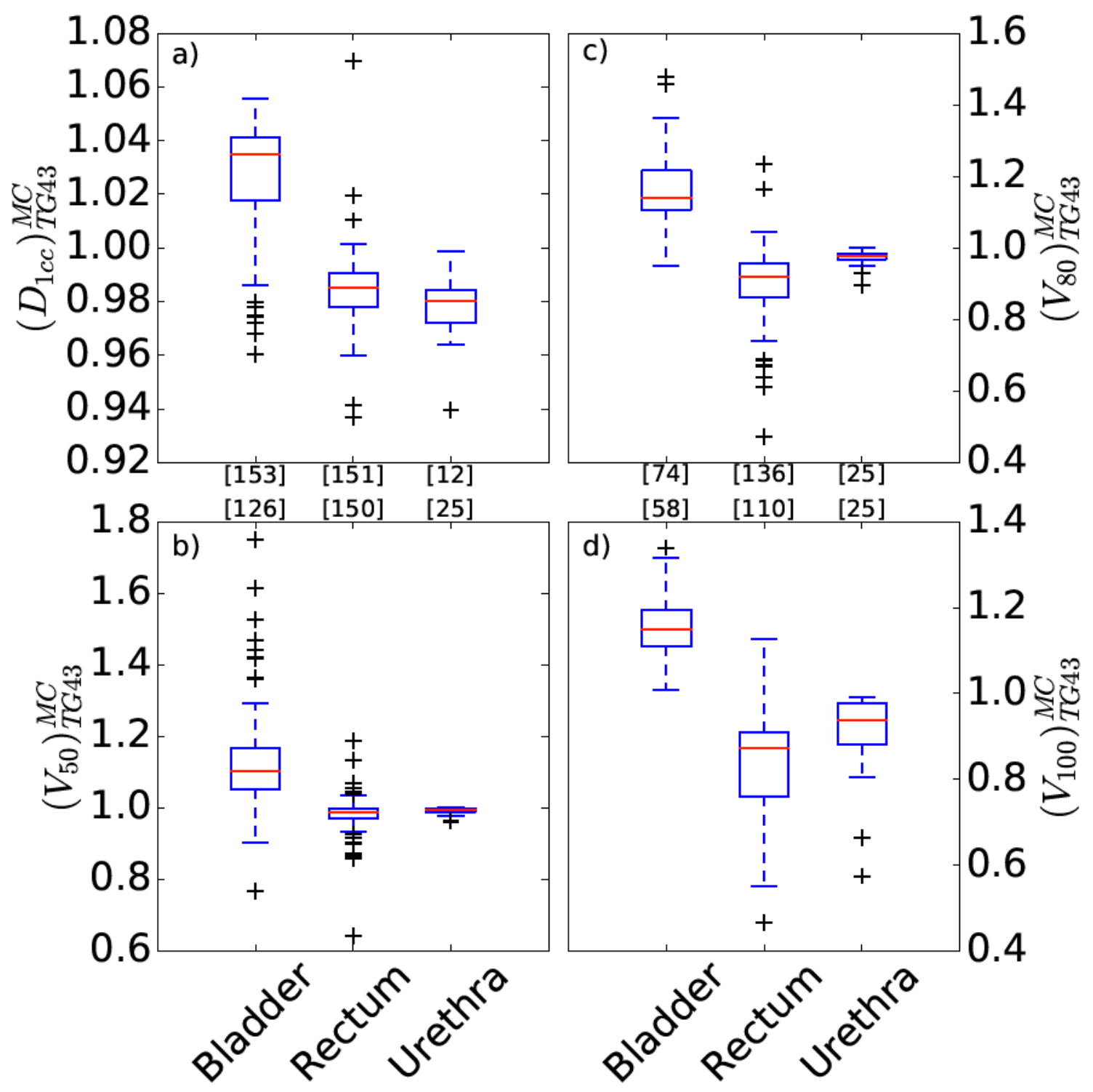

Figure 3.11: Box and whiskers plot $M C$ calculated distribution of organs-at-risk dosevolume metrics normalized to TG43. Numbers in square brackets at the center of the figure represent number of each patients included in the corresponding box-plot (out of the initial $\mathrm{N}_{\text {Bladder }}=154, \mathrm{~N}_{\text {Rectum }}=152, \mathrm{~N}_{\text {Urethra }}=12$ ). 
signed the same tissue composition as prostate meaning that the photon attenuation and photon energy-absorption is slightly less than water. Discrepancies in urethral dose-volume metrics between $M C$ and TG43 are mostly attributed to inter-seed effects; unlike the other OAR, urethral proximity to seeds within the treatment volume makes the organ subject to inter-seed effects. Low-dose streaks present due to photon attenuation by neighboring seeds (previously described in $§ 3.1$ and illustrated in Figure 3.1) may overlap with the urethra, acting as a "shadow" from the radiation. Urethral $D_{1 c c}$ is overestimated by $2.23 \%$ by $T G 43$, on average. Urethral $V_{50}, V_{80}$, and $V_{100}$, are overestimated by $T G 43$ by $0.861 \%, 2.69 \%$, and $9.62 \%$, on average, respectively.

\subsection{Radiobiological Modeling}

Figure 3.12 presents histograms of biologically effective dose (BED) and tumor control probability (TCP) for the patient cohort calculated with TG43 and MC, where voxels with doses less than 110 Gy are discarded from the prostate volume, as done in the literature ${ }^{53}$. With this method, it can be observed that BED values are close for all patients given the small deviation from the average BED, for both TG43 and MC calculation methods. Utilizing this model with the given parameters estimates a high TCP for the majority of the cohort. With the consideration of tissue heterogeneities and inter-seed effects in $M C$, there is not much variability in BED and TCP distribution compared to TG43; the most notable differences between the two dose calculation schemes is observed in patients with BED greater than one standard deviation from the mean.

The use of a 110 Gy dose cut-off is justified by King et al. ${ }^{53}$ for use with the protocol outlined in the TG-43 report, where tissue heterogeneities, that may result in low-dose regions due to large calcifications, are not considered. To illustrate the 

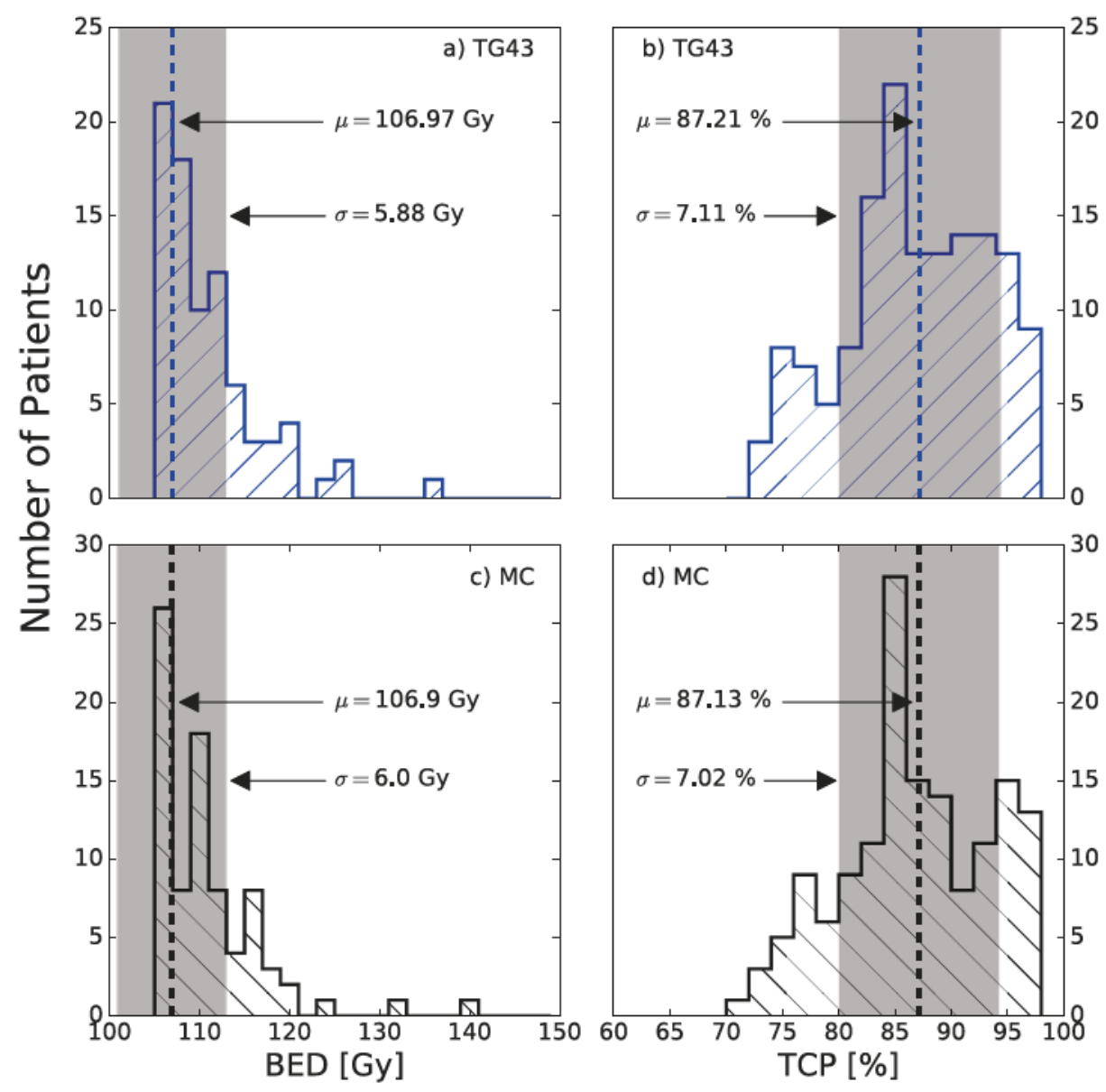

Figure 3.12: Histogram of (a, c) biologically effective dose (BED) and (b, d) tumor control probability (TCP) for 110 Gy dose cut-off for dose distributions calculated with (blue histogram) TG43 and (black histogram) $M C$ for all patients $(N=157)$. Average BED and TCP with one standard deviation overlaid. 
fraction of voxels discarded with the 110 Gy dose cut-off, Figure 3.13 presents cumulative DVH for 5 patient prostates calculated with $M C$, where the 110 Gy dose cut-off is shown to remove a non-negligible fraction of prostate voxels. While DVHs lack spatial information, the assumption that these discarded voxels are at the periphery of the organ is untrue for specific patient cases. Therefore, it is imperative to take into consideration these low-dose voxels since they can lead to compromised BED and TCP under this model.

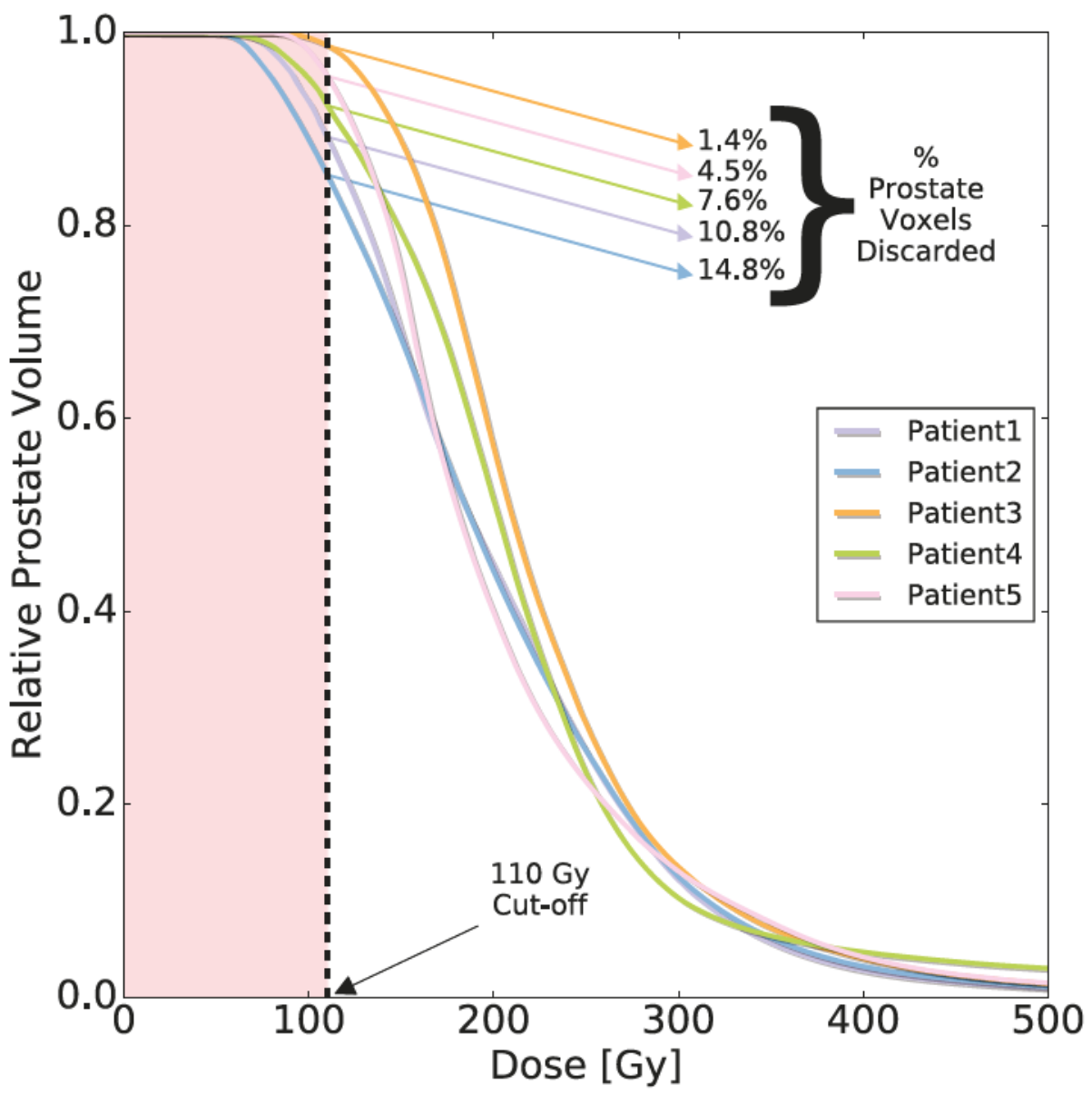

Figure 3.13: Cumulative dose-volume histograms calculated with $M C$ for 5 patient prostates. Dashed vertical line with shaded red region represents dose threshold (110 Gy) where voxels doses less than this are discarded. Percentage of total prostate voxels discarded for each patient overlaid.

Figure 3.14 presents histograms of biologically effective dose (BED) and tumor control probability (TCP) for the patient cohort calculated with TG43 and MC, where 
voxels with doses less than 9.43 Gy are discarded from the prostate volume. This histogram illustrates a lower patient average BED (about -23 Gy), with a wider spread of BED values (about +12 Gy) compared to the 110 Gy cut-off. The largest difference between the 110 Gy and 9.43 Gy cut-off approaches appears for computed TCP values, where the consideration of low-dose voxels is shown to diminish TCP across the entire patient cohort. According to the TCP model, tumor cell survival after the treatment compromises a successful outcome; prostate under-dosage considered in $M C$ may lead to tumor cell survival, so the result is as expected for this model. Compared to Figure 3.12, BED and TCP distributions over the patient cohort are also similar when using dose distributions calculated with TG43 and MC.

\subsection{Outcome Study}

Radiation toxicities scored according to the CTCAE guidelines are summarized in Table 3.3. Generally, most patients received treatments that were free of severe adverse side-effects. The most common side-effect to the PIPB treatment observed is grade 1 urinary complication. Treatment-induced prostate edema is a very common occurrence for men receiving brachytherapy treatments; difficulty or painful urination (dysuria) is caused by the restriction of the urethra that runs through the temporarilyenlarged prostate shortly after the treatment. Patients treated with Flomax (Tamsulosin) were generally relieved of these symptoms. Complications such as rectal bleeding and erectile dysfunction were also reported retrospectively, but at a lower frequency relative to urinary complications.

Figure 3.15 presents urinary, rectal, and erectile complications of grade 0 to 3 related to absolute dose metrics for prostate $D_{90}$ (urinary and erectile complications), and rectal $D_{1 c c}$ (rectal complications). Similarly, Figure 3.16 presents the same com- 

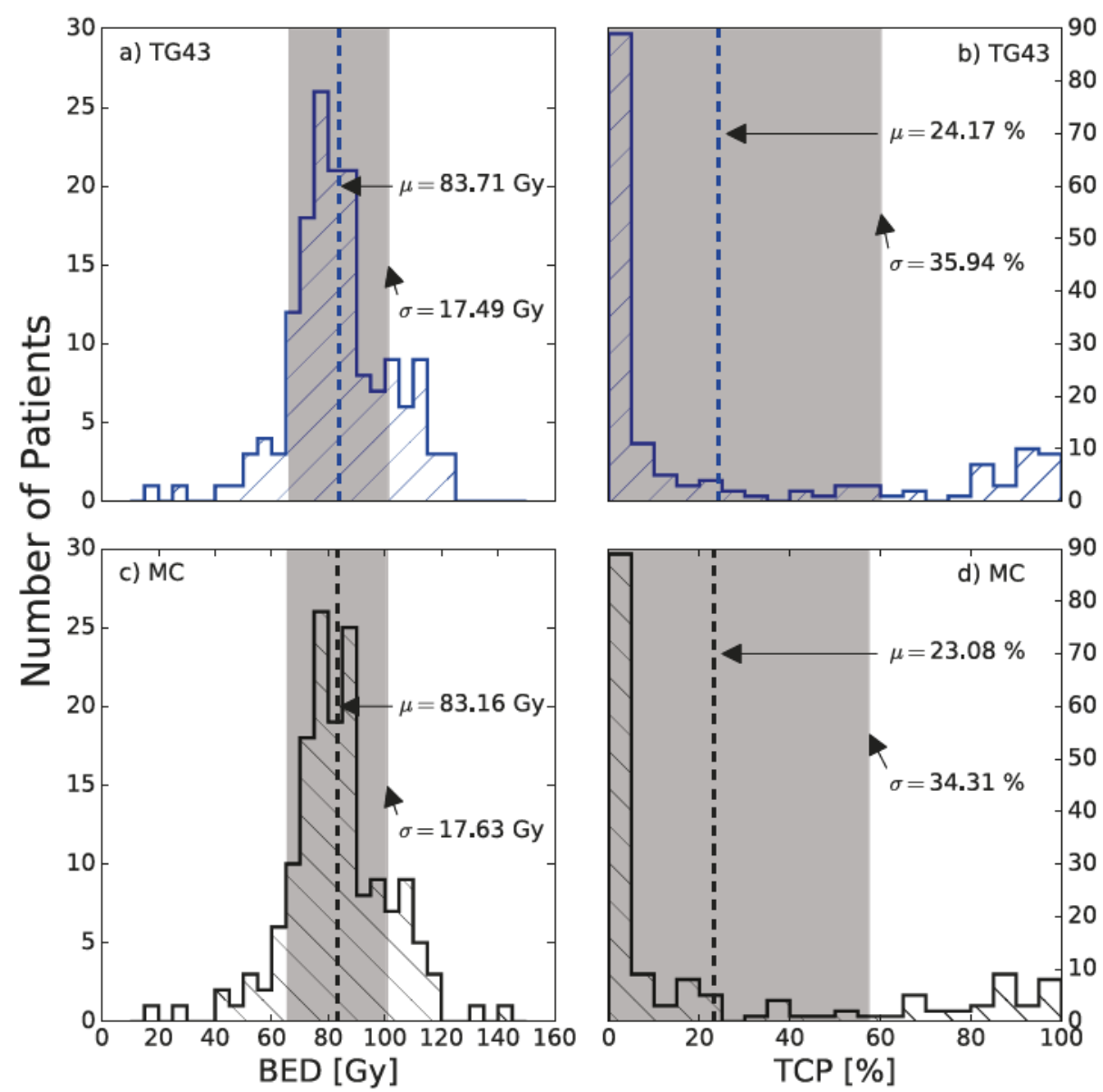

Figure 3.14: Histogram of $(\mathrm{a}, \mathrm{c})$ biologically effective dose (BED) and (b, d) tumor control probability (TCP) for 9.43 Gy dose cut-off for dose distributions calculated with (blue histogram) TG43 and (black histogram) $M C$ for all patients $(N=157)$. Average BED and TCP with one standard deviation overlaid.

Table 3.3: Summary of treatment toxicity outcomes for 157 patients in the follow-up database. Number of patients and percentage of entire cohort with each outcome reported for each radiation toxicity. Followup dates range from November 19 ${ }^{\text {th }}, 2007$ to June $6^{\text {th }}, 2016$.

\begin{tabular}{|c|c|c|c|}
\hline \multirow[b]{2}{*}{ Outcome } & \multicolumn{3}{|c|}{ Radiation Toxicity } \\
\hline & Erectile & Rectal & Urinary \\
\hline None & $120(76 \%)$ & $125(80 \%)$ & $39(25 \%)$ \\
\hline Grade 1 & $22(14 \%)$ & $18(11 \%)$ & $85(54 \%)$ \\
\hline Grade 2 & $9(6 \%)$ & $11(7 \%)$ & $31(20 \%)$ \\
\hline Grade 3 & $4(3 \%)$ & $1(<1 \%)$ & $0(0 \%)$ \\
\hline Missing & $2(1 \%)$ & $2(1 \%)$ & $2(1 \%)$ \\
\hline
\end{tabular}




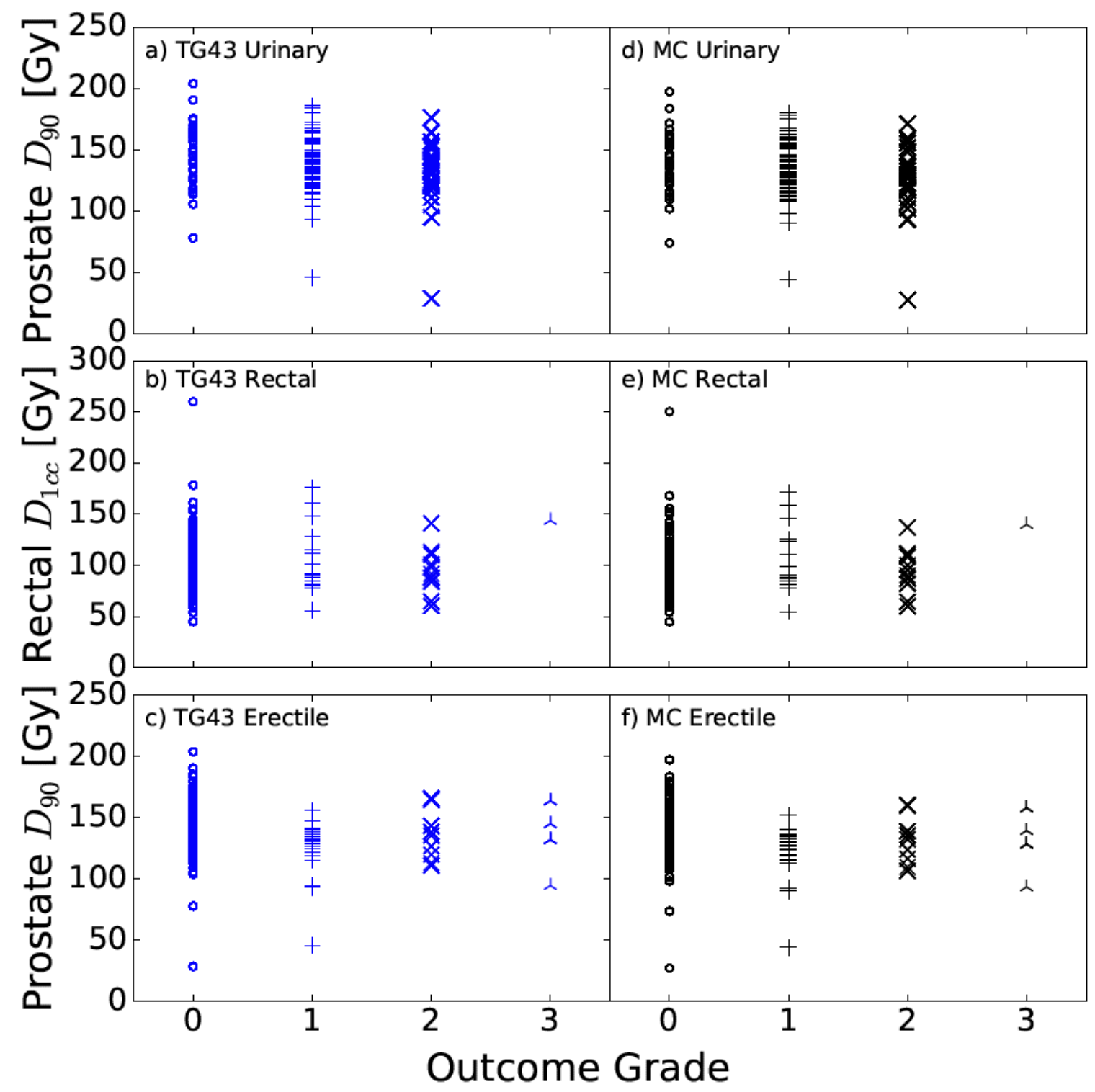

Figure 3.15: Comparison of radiation toxicity outcomes related to dose metrics calculated with TG43 and $M C$ for urinary $\left(N_{\text {Urinary }}=155\right)$, rectal $\left(N_{\text {Rectal }}=149\right)$, and erectile complications $\left(N_{\text {Erectile }}=155\right)$. Dose [Gy] for respective dose metric plotted against outcome grade. 
plications related to absolute volume metrics: prostate $V_{200}$ (urinary and erectile complications), and rectal $V_{100}$ (rectal complications). Neither absolute dose nor volume metrics calculated with $T G 43$ or $M C$ were found to have any correlation or act as a predictor for the radiation toxicity grade for the patient cohort in this study. Additionally, high grade radiation toxicities were not found to be dependent on anomalous differences in dose distributions pertaining to the target, and organs-at-risk derived by either dose calculation scheme. Lastly, the number of patients that developed a grade 3 toxicity was found to be very low $(N=5)$.

Figure 3.17 presents urinary, rectal, and erectile complications of grade 0 to 3 related to $T G 43$ normalized $M C$ dose and volume metrics. Exploring the same set of dose-volume metrics as Figure 3.15 and 3.16, similar conclusions may be drawn: namely, the ratio of dose-volume metrics calculated with the two simulation schemes does not provide any correlation nor acts as a predictor for radiation toxicity grade. 


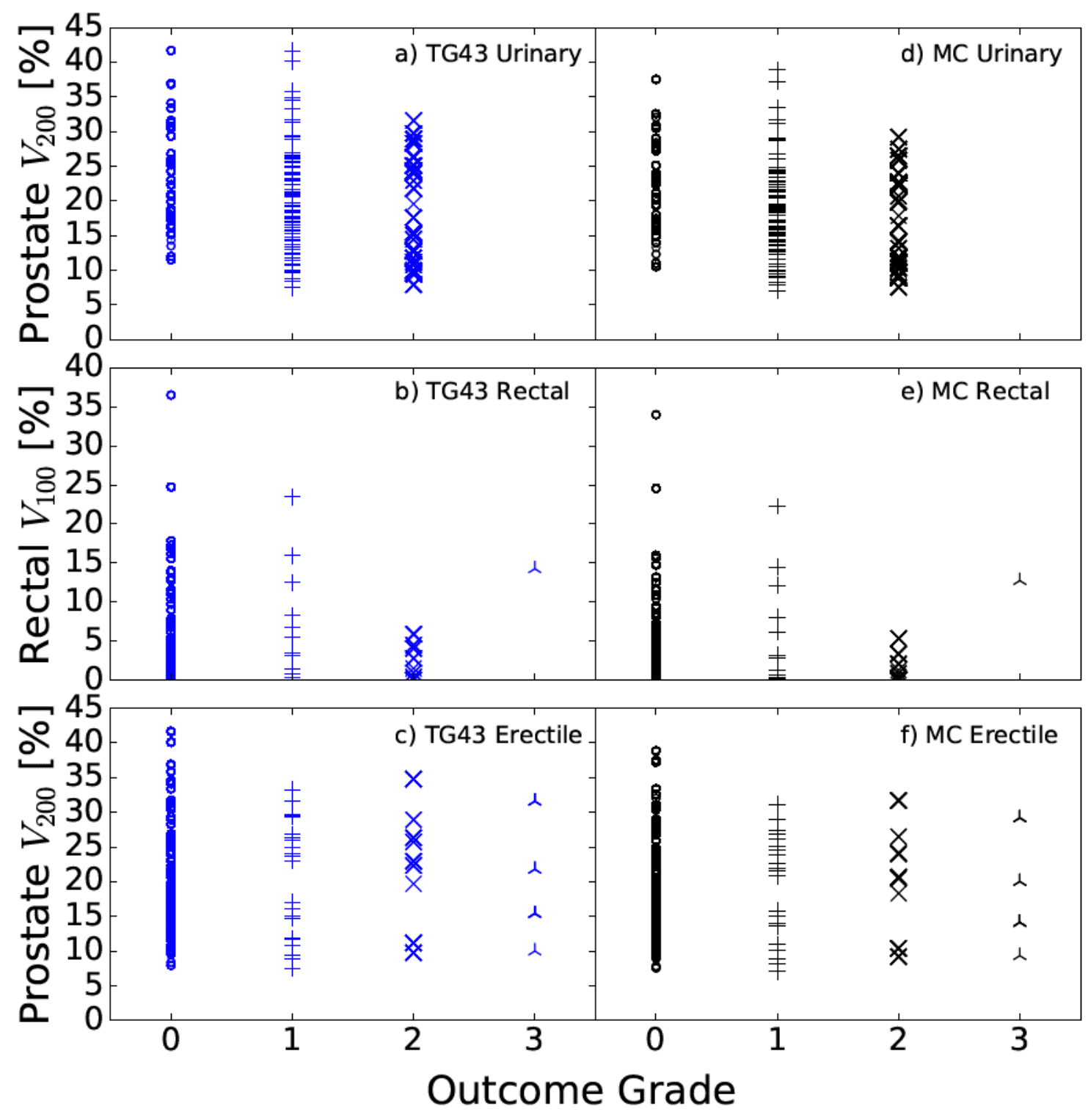

Figure 3.16: Comparison of radiation toxicity outcomes related to volume metrics calculated with TG43 and $M C$ for urinary $\left(N_{\text {Urinary }}=155\right)$, rectal $\left(N_{\text {Rectal }}=149\right)$, and erectile complications $\left(N_{\text {Erectile }}=155\right)$. Fractional volume [\%] for respective volume metric plotted against outcome grade. 


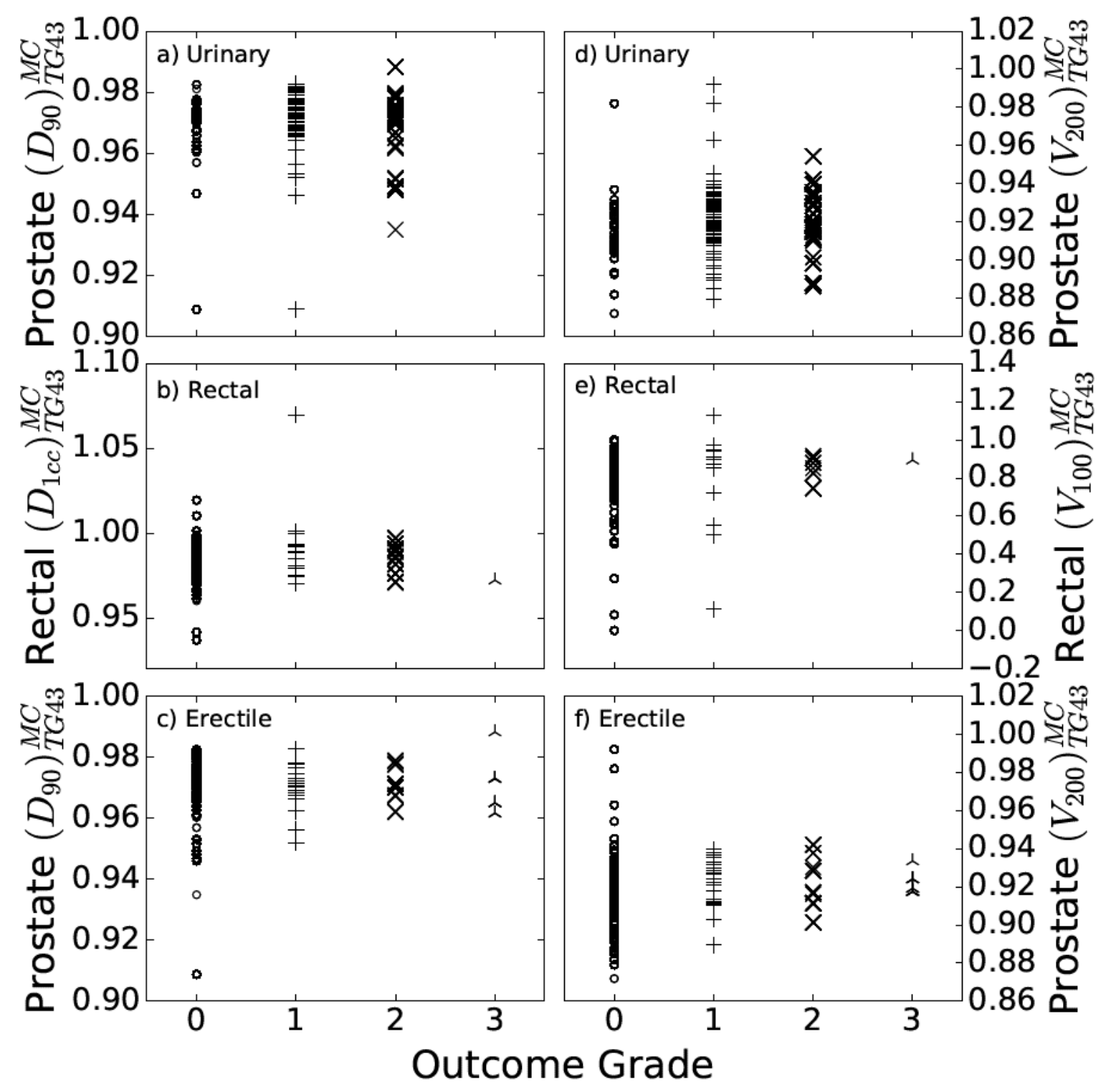

Figure 3.17: Comparison of radiation toxicity outcomes related to normalized dosevolume metrics for urinary $\left(N_{\text {Urinary }}=155\right)$, rectal $\left(N_{\text {Rectal }}=122\right)$, and erectile complications $\left(N_{\text {Erectile }}=155\right)$. Calculations with $M C$ normalized to $T G 43$ for respective dose-volume metrics plotted against outcome grade. 


\section{Chapter 4}

\section{Discussion}

\subsection{Dosimetry}

\subsubsection{Prostate}

Similar studies in the literature investigate the effects of tissue heterogeneities and inter-seed effects compared to the TG-43 formalism for $\mathrm{I}^{125}$ brachytherapy. Differences between applications of model-based approaches within the literature may be dependent on the choice of prostate tissue composition ${ }^{62}$, seed density ${ }^{23}$, prostate size $^{23}$, amount of calcification tissue $e^{25,28}$ within the prostate, and seed model ${ }^{63}$.

Landry et al. ${ }^{62}$ investigated the effect of tissue composition and mass density on prostate $D_{90}$, for an ${ }^{125}$ | brachytherapy source, ignoring inter-seed effects. Using three prostate tissue compositions taken from Woodard and White ${ }^{20}$ (Prostate A, tissue composition used in this study), ICRP ${ }^{21}$ (Prostate $B$, equivalent to Prostate $A$ ), and ICRP ${ }^{64}$ (Prostate $C$, equivalent to average male soft tissue used in this study from ICRU $\left.{ }^{22}\right)$, and using mass densities set to unity $(\rho=1)$ or CT derived values, they score $D_{w, m}$ (radiation transport in medium with absorbed dose scored in water) and $D_{w, w}$ (TG-43) for 4 patients. When neglecting density effects (setting $\rho=1$ ), they find Prostate $A$ to be water equivalent with no difference in $D_{90}$ when compared to TG-43 $\left(\left[D_{90}^{\text {Prostate } A}-D_{90}^{T G-43}\right] / D_{90}^{T G-43}\right)$; Prostate $C$ is reported to overestimate 
prostate $D_{90}$ by $3.2 \%$ compared to TG- 43 . This can be understood as the difference in photon attenuation resulting from differences in mass attenuation coefficients between Prostate $A\left((\mu / \rho)_{w, 28}^{m} \mathrm{keV}=0.998\right)$ and Prostate $C\left((\mu / \rho)_{w, 28 \mathrm{keV}}^{m}=0.953\right)$. Photon attenuation is decreased in Prostate $C$ compared to Prostate $A$ resulting in a higher photon fluence. Since dose to water is scored, increased photon fluence leads to higher dose deposition. While dose to medium was not explored in this study, the impact of tissue composition on prostate $D_{90}$ is illustrated through changes in photon attenuation of different media.

Comparing the results of Landry et al. ${ }^{62}$ to the study presented herein, CT derived mass densities for prostate composition Prostate $A$ are used, and $D_{w, m}$ is converted to $D_{m, m}$ using the mass-energy absorption coefficient ratio of medium to water at the mean energy of ${ }^{125} \mathrm{I}\left(\left(\mu_{e n} / \rho\right)_{m, 28}^{w} \mathrm{keV}=0.993\right)^{6,62}$. Landry et al. ${ }^{62}$ find prostate $D_{90}$ is overestimated by $1.3 \%$ by TG-43 ([1- $\left.\left.D_{90}^{T G-43} / D_{90}^{m, m}\right] \cdot 100 \%\right)$, compared to the overestimation found in this study of $3.0 \%$. Discrepancies between the two arise from the following factors: converting $D_{w, m}$ to $D_{m, m}$ using $\left(\mu_{e n} / \rho\right)_{w, 28}^{m} \mathrm{keV}$ ignores the variation of $\left(\mu_{e n} / \rho\right)_{w}^{m}$ with photon energy; ignoring inter-seed effects can lead to decreases in $D_{90}$ of about $4 \%{ }^{23,24,26}$. Additionally, Landry et al. ${ }^{62}$ had a small patient group (4 patients), which does not provide adequate patient variability and statistical certainty.

Carrier et al. ${ }^{23}$ performed a pre-implant investigation on the effect of ${ }^{125}$ I seed density and source strength on prostate dose-volume metrics for two prostate volumes: $26 \mathrm{~cm}^{3}$ and $59 \mathrm{~cm}^{3}$. By comparing dose calculations in a water phantom with inter-seed effects off (SMC, similar to TG43) and on (MCW, similar to MC with all media set to water), they concluded that inter-seed attenuation is increased with increasing seed density. Higher seed densities imply smaller inter-seed distances that lead to increased photon-seed interactions that produce discrepancies between dose 
calculations performed with $S M C$ and $M C W$. The larger prostate volume is shown to have a bigger inter-seed effect, attributed to higher global attenuation for the larger prostate volume. Increasing source strength is shown to decrease inter-seed attenuation in both prostate volumes, due to fewer implanted seeds (lower seed density). SMC overestimates prostate $D_{90}$ by approximately $1 \%$ - $6 \%$ compared to $M C W$ for both prostate volumes; prostate $V_{200}$ is overestimated by approximately $1 \%-9 \%$ and $1 \%-6 \%$ for the $59 \mathrm{~cm}^{3}$ and $26 \mathrm{~cm}^{3}$ prostate volumes, respectively.

Carrier et al. ${ }^{23}$ included an additional calculation scheme that considers tissue heterogeneities (MCP, similar to $M C)$, i.e. modeling prostate tissue. They reported a $4.4 \%$ and $4.8 \%$ overestimation of prostate $D_{90}$ by only considering a homogeneous water phantom for the $26 \mathrm{~cm}^{3}$ and $59 \mathrm{~cm}^{3}$ prostate volumes, respectively. Overall, comparing $M C P$ to clinical calculations based on TG-43U1 (generating geometry function using line-source approximation), they reported TG-43U1 as overestimating prostate $D_{90}$ by $5.8 \%-12.8 \%$, depending on prostate volume and seed density. These values show larger dose overestimation by TG43 compared to the current study, on average; however, considering the range of prostate $D_{90}$ overestimation by $M C$ in this study $(1.2 \%-9.1 \%)$, there is a small overlap with values in Carrier et al. ${ }^{23}$ Differences between reported values could be due to Carrier et al. ${ }^{23} \mathrm{com}$ paring model-based calculations to calculations based on TG-43U1, as opposed to a scheme equivalent to TG43, that accounts for about a 1\% underestimation by TG43U1. In a similar study, Carrier et al. ${ }^{24}$ report an average overestimation by TG-43 of prostate $D_{90}$ and $V_{100}$ by $6.5 \%$ and $2.1 \%$, respectively, comparing two schemes similar to TG43 and MC. Although the average overestimation of $D_{90}$ is higher than the average from this study, the reported $V_{100}$ overestimation by TG-43 are in agreement.

Fekete et al. ${ }^{25}$ investigated the effect of tissue heterogeneities and inter-seed 
effects on prostate dose-volume metrics, compared to traditional TG-43, for a cohort of patients with prostatic calcifications. Comparing two calculation methods similar to $M C$ and TG43, they report an average overestimation by TG-43 of prostate $D_{90}$ and $V_{100}$ of $6.4 \%$ and $2.6 \%$, respectively. For an extreme case, a patient with a large calcification, prostate $D_{90}$ was overestimated by $14.1 \%$ by TG-43. While reported average prostate $V_{100}$ were in agreement with this study within statistical uncertainties, prostate $D_{90}$ metrics were not in agreement. Fekete et al. ${ }^{25}$ uses a patient cohort of exclusively calcification patients, compared to the non-calcification patients included in this study, so larger overestimations of prostate $D_{90}$ are expected by Fekete et al. ${ }^{25}$. They also find calcifications responsible for reductions in dose of up to $45 \%$ in the surrounding prostate medium compared to TG- 43 , a finding that is consistent with this study. Furthermore, Fekete et al. ${ }^{25}$ observe a correlation between fraction of calcification in the prostate and $D_{90}$ overestimation by TG-43, similar to Figure 3.9(a) in this study, meaning there is an agreement in the dose-volume metric decrease effect attributable to prostatic calcifications.

Mason et al. ${ }^{28}$ investigated the impact of calcifications on prostate dose-volume metrics for 10 patients with a mean fraction of prostate voxels assigned to calcification of about $0.9 \%$; they report an average decrease of prostate $D_{90}$ and $V_{100}$ of $4.5 \%$ and $2.1 \%$, respectively, compared to TG-43 based dose calculations. For the most extreme case, an overestimation by TG-43 of prostate $D_{90}$ and $V_{100}$ of $7.4 \%$ and $4.8 \%$ was reported, respectively. As an exercise, values from Mason et al. ${ }^{28}$ will be compared with the results of the first-degree polynomial fit in Figure 3.9, which gives an expected decrease of prostate $D_{90}$ and $V_{100}$ of $4.7 \%$ and $3.8 \%$, for $0.9 \%$ prostate volume assigned calcification, respectively. It should be noted that the polynomial fit is performed for all patients with and without calcifications. Removing patients with fractional prostate voxels assigned calcification of less than $0.5 \%$ (corresponding to the lower bound of Mason et al. ${ }^{28}$ calcification patient cohort), the coefficient of de- 
termination is improved $\left(R_{D_{90}}^{2}=0.853, R_{V_{100}}^{2}=0.767\right)$ and an expected decrease of $D_{90}$ and $V_{100}$ of $4.7 \%$ and $3.9 \%$ is computed, respectively. Decreases in prostate $D_{90}$ between reported values and expected values from the polynomial fit are in excellent agreement, while decrease in $V_{100}$ are not. Performing the same analysis for the study by Fekete et al. ${ }^{25}$, where the mean fraction of prostate voxels assigned to calcification is $1.7 \%$, the polynomial fit gives expected decreases of prostate $D_{90}$ and $V_{100}$ of $6.3 \%$ and $5.6 \%$, respectively. Compared to values reported by Fekete et al. ${ }^{25}$ of $6.4 \%$ and $2.6 \%$ for prostate $D_{90}$ and $V_{100}$, respectively, expected values are in good agreement for prostate $D_{90}$ but overestimate the reduction for $V_{100}$.

Generally a consensus between works in the literature (discussed above) and this study is reached in concluding prostate dose-volume metrics are overestimated by clinical TG-43 calculations. The degree of overestimation has been shown to be greatly influenced by the presence of prostatic calcifications, in addition to a variety of other factors. Implantation technique differs from center-to-center, and depending on source strengths used this may lead to varying number of seeds implanted for a given procedure. As shown by Carrier et al. ${ }^{23}$, inter-seed effects account for a decrease of prostate $D_{90}$ of $1 \%$ - $6 \%$ compared to TG-43 depending on source strengths and implanted seed densities. Differences in seed models used at various centers may also contribute to differences in dose distributions. Afsharpour et al. ${ }^{63}$ investigated the effect of different seed geometries, radiopaque markers, and source encapsulations on prostate $D_{90}$ dose reduction by MC compared to TG-43 for four ${ }^{125} \mid$ and two ${ }^{103} \mathrm{Pd}$ seeds using two patient cases. Differences of up to $4.0 \%$ in $D_{90}$ reduction by MC are reported depending on seed model. Since low energy photons show large variability in mass-energy absorption coeffecient depending on tissue composition, differences in photon cross-sections used can lead to variability in computed dose distributions. The numbers of patients investigated by other works were low $(\mathrm{N}<45)$ compared to this study $(\mathrm{N}=157)$, so lack of patient variability and sample size result 
in differing degrees of statistical certainty.

\subsubsection{Organs-at-Risk}

\section{Urethra}

Works in the literature by Fekete et al. ${ }^{25}$ and Mason et al. ${ }^{28}$ report urethral $D_{10}$ overestimation by TG-43 using dose calculations derived using a model-based approach similar to $M C$ and a scheme similar to TG43. Urethra, however was assigned as Average Male Soft Tissue (same composition as Table 2.2) as opposed to Prostate Tissue. They report an average urethral $D_{10}$ overestimation by TG- 43 of $4.5 \%{ }^{25}$ and $3.5 \%{ }^{28}$. In this study, urethral $D_{0.1 c c}$ and $D_{1 c c}$ are overestimated by TG-43 by $2.7 \%$ $\pm 1.5 \%$ and $2.3 \% \pm 1.5 \%$, on average. Values reported by Fekete et al. ${ }^{25}$, Mason et al. ${ }^{28}$ and the current study are not comparable due to differences in tissue assignment in the urethra and choice of urethral dose metrics. While there is no explicit urethral tissue composition in the literature, the choice between Prostate Tissue used in this study and the urethra composition used by Fekete et al. ${ }^{25}$ and Mason et al. ${ }^{28}$ have been investigated in an abstract by the author ${ }^{65}$. It was found that Average Male Soft Tissue decreases urethral $D_{0.1 c c}$ and $D_{1 c c}$ compared to Prostate Tissue by an additional $6 \%$, on average. The average urethral volume in this study is $1.0 \mathrm{~cm}^{3}$ $\pm 0.4 \mathrm{~cm}^{3}(\mathrm{~N}=25)$, making $D_{0.1 c c}$ a good surrogate for $D_{10}$; directly comparing the two values, this study finding of $2.7 \% \pm 1.5 \%$ overestimation by TG-43 is in agreement within statistical uncertainty to the $4.5 \%$ and $3.5 \%$ overestimation reported in the literature. Applying a simplified raw correction to account for the different urethral tissue composition, values from our study are increased by $6 \%$; an overestimation of $2.9 \% \pm 1.6 \%$ by TG-43 is calculated, resulting in better agreement with results from the literature. 
Urethral visualization in post-implant CT imaging is poor, therefore the use of urinary catheter with contrast may be employed, which leads to perturbation of the true anatomical urethral volume and issues with assigning voxel mass densities using CT number for derivation of virtual patient phantoms (similar to issues discussed in §2.2.4). Patients with contrast present in the urinary catheter must be assigned a nominal mass density $\left(\rho=1.0 \mathrm{~g} / \mathrm{cm}^{3}\right)$ that may lead to underestimations in urethral dose-volume metrics. The minor anatomical inaccuracy of urethral volume perturbation via urinary catheter can be assumed as an additional uncertainty in the various uncertainties in brachytherapy dose calculations and treatments.

\section{Rectum}

Works by Mason et al. ${ }^{28}$ and Carrier et al. ${ }^{24}$ reported rectal dose-volume overestimations by TG-43 rectal $D_{2 c c}{ }^{28}$ and $V_{100}{ }^{24}$ of $10.5 \%$ and $10.9 \%$, respectively, for a small cohort of patients $(\mathrm{N}<30)$. The current study reports an overestimation of rectal $D_{1 c c}$ and $V_{100}$ of $1.6 \% \pm 1.3 \%(\mathrm{~N}=151)$ and $17 \% \pm 11 \%(\mathrm{~N}=110)$. Mason et al. ${ }^{28}$ assigned rectum as Average Male Soft Tissue while Carrier et al. ${ }^{24}$ assigns Rectal Tissue (same composition as Table 2.2). Rectal $V_{100}{ }^{24}$ is in good agreement within statistical uncertainty with values reported in this study. Rectal $D_{2 c c}{ }^{28}$ is found to be overestimated by TG-43. While not being directly comparable to rectal $D_{1 c c}$ in the current study due to difference in tissue assignment and dose metric, rectal dose metrics are found to be generally overestimated with TG43. Average Male Soft Tissue has a high adipose content that results in a mass attenuation coefficient $7 \%$ lower than Rectal Tissue (at $28 \mathrm{keV}$ ), explaining the large discrepancies in the magnitude of overestimation. Rectal dose-volume metrics in this study took a wide range of values, including some that reported underestimations by TG-43. Many inter-patient differences such as rectal-prostate distances, rectal-seed distances, and rectal size 
may result in large variability in dose-volume discrepancies between MC and TG43.

Differences in rectal contouring between different centers, i.e. contouring a semi-circular rectal wall adjacent to the prostate versus the entire rectum, can result in differences in volume metrics between reported values. The latter method, while including the entire rectal volume, may include dose to discarded metabolic waste in the retrospective patient model. The impact of the differing methods has unknown clinical significance.

\section{Bladder}

Carrier et al. ${ }^{24}$ reported an overall average overestimation of bladder $V_{100}$ of $15.8 \%$ by TG-43 based calculations. However, some patients with overall underestimation of $V_{100}$ by TG-43 based calculations were also found. Separating tissue and inter-seed effects on dosimetric differences, they report bladder underestimation by TG-43 due to the tissue effect of $11.1 \%$ on average, and bladder overestimation due to inter-seed effects of $19.1 \%$, on average. Comparatively, this current study reports bladder $V_{100}$ underestimation by TG-43 of $16 \% \pm 7 \%$, on average. While these values are generally not in agreement, the inter-patient variability, including, but not limited to, differences in source strength, bladder-prostate distance, and bladder-seed distances lead to uncertainty in agreeing upon a result. Similar to the issue in rectal contouring, differences in bladder contouring, i.e. contouring the entire structure versus the wall adjacent to the prostate, have unknown effects on dosimetric analysis.

\subsubsection{General Comments}

Retrospective studies of this nature have some inherent shortcomings when deriving patient specific models and computing dose distributions. Post-implant dosime- 
try only considers the static patient anatomy and seed geometry at one time, not considering any dynamic effects such as procedure-induced prostate edema, or the migration of seeds over time. Edema increases prostate volume immediately after the procedure for up to 30 days $^{6}$ (large fraction of total dose delivered in this timeframe) leading to an increase in inter-source distances. If post-implant dosimetry is performed after the edema has mostly subsided, an overestimation of target dose ${ }^{54}$ is expected. A study by Taussky et al. ${ }^{66}$ shows statistically significant changes in derived prostate $D_{90}$ due to treatment induced edema. The study concludes however that prostate edema plays a clinically-insignificant role for good quality implants. Studies by Taschereau et al. ${ }^{67}$ and Gao et al. ${ }^{68}$ show the effect of seed migration on dosimetric analysis and concluded that not accounting for seed migration may result in significant differences in dose distributions. They show seeds tend to cluster leading to higher local seed densities resulting in larger inter-seed effects.

The accuracy of virtual patient models derived using a contour-guided voxel-byvoxel approach is dependent on the accuracy of the physician-defined target and organs-at-risk contours. Since modeled organ positions and volumes are dependent on contour data, discrepancies in the contoured organ volume and "real" organ volume can result in inaccurate patient models and dose distributions. Dosimetric analyses using dose-volume histograms is dependent on organ volumes as defined by contours and shows inherent sensitivity of interpolated dose-volume metrics with contour variability. Gao et al. ${ }^{69}$ investigated inter- and intra-physician variability in CTbased prostate volume delineation among 6 physician against a gold standard defined by a multi-disciplinary group of experts based on The Visible Human data set ${ }^{70}$. They conclude that physician delineated prostate contours were 30\% larger than the "real" organ volume with only $84 \%$ coverage of the gold standard, ie. physicians are overestimating prostate size in addition to contouring non-prostatic regions. The intra-physician variation ranged from $2 \%$ to $8 \%$ standard deviation of the mean, with 
an $18.8 \%$ inter-physician standard deviation of the mean. This leads to increased uncertainties in physician-defined contours, appending to the list of uncertainties for dose calculations and dosimetric analysis.

As discussed previously, sensitivity to tissue compositions results in differences in derived dose distributions and dosimetric quantities. Inter-patient variability in tissue composition however is not taken into consideration in this dosimetric study.

\subsection{Radiobiological Modeling}

This study has applied a simple radiobiological model using dose distributions computed with advanced MBDCA to derive two radiobiological indicies, biologically effective dose (BED), and tumor control probability (TCP). Afsharpour et al. ${ }^{71}$ use a Monte Carlo approach and apply it to the radiobiological model by Dale and Jones ${ }^{35}$ (also used in the current study) to estimate $\alpha / \beta$. This involved using post-implant prostate $D_{90}$ derived using MC, in addition to the voxel-by-voxel approach, to compute BED. While the methodology for the voxel-by-voxel approach is unclear (ie. the approach taken for voxels with doses that gave negative $T_{\text {eff }}$ depending on the choice of $\alpha$ ), they underscore the sensitivity of BED to choice of radiobiological modeling parameters. The current study is in agreement with Afsharpour et al. ${ }^{71}$ in stating the importance of considering tissue heterogeneities and inter-seed effects in dose calculation models. While there is no agreement in the literature for specific values for $\alpha / \beta$, especially across different prostate cancer cell lines, there is a general consensus that $\alpha / \beta$ for prostate cancer is low ${ }^{54}$. This thesis does not investigate various radiobiological models, or sensitivity to radiobiological modeling parameters found in the literature, but instead outlines the importance and challenges with considering dosimetry using a model-based calculation paradigm. Tissue heterogenities and inter-seed effects have been shown to give rise to areas of under-dosage in the 
prostate not considered with the TG-43 formalism. These modeling considerations result in more accurate dose distributions that can be used for analysis with current radiobiological models.

\subsection{Outcomes Study}

Some works in the literature explore the usage of dosimetric quantities, based on the TG-43 formalism, as a predictor for disease free survival (DFS). Stock et al. ${ }^{72}$ reported on prostate $D_{90}$ as a predictive factor for DFS, concluding that patients that receive $D_{90}$ lower than 140 Gy had a 4-year DFS rate of $68 \%$ compared to those receiving 140 Gy or higher (92\% DFS, $p=0.02)$. Additionally, they show that prostate $D_{90}$ is a better predictive factor compared to PSA, Gleason Score, and TNM-staging $(p<0.001)$. Prostate $D_{90}$ may be understood as a predictor of implant quality, with a bad implant ( $D_{90}<140 \mathrm{~Gy}$ ) leading to poor tumor control resulting in lower DFS rate. Other studies ${ }^{73-76}$ have confirmed findings by Stock et al. ${ }^{72}$, with some showing prostate $V_{100}$ as a better predictor of DFS than $D_{90}$. Studies by Morris et al. ${ }^{77}$ and Yorozu et al. ${ }^{78}$ have failed to draw a correlation between dosimetric quantities and DFS. Another study by Stock et al. ${ }^{79}$ has shown biologically effective dose (BED) as a significant predictor of DFS $(p<0.0001)$. While dosimetric quantities such as prostate $D_{90}, V_{100}$, and BED are shown to correlate well with DFS, no published study has been able to correlate these quantities with radiation toxicity outcomes. Furthermore, no work in the literature has attempted to correlate dosimetric quantities derived using a model-based approach with any clinical endpoint. This study aimed to utilize a more accurate model-based approach to dose calculations to compute dosimetric quantities that, in theory, were better predictors of treatment outcomes and DFS compared to the TG-43 formalism. However, lack of patients follow-up data, few high grade radiation toxicities, and few PSA failures lead to difficulties in drawing statistically significant correlations between dosimetric values (computed with either 
MC or TG43) and clinical endpoints. 


\section{Chapter 5}

\section{Conclusions and Future Outlook}

This study presented a dosimetric comparison between the current clinical protocol of dose calculations outlined in TG-43 and an advanced model-based approach of dose calculations that take into consideration tissue heterogeneities and inter-seed effects. Results presented for the prostate, bladder, rectum, and urethra showed differences between TG-43 and the model-based approach. Prostate, rectal, and urethral dosimetric quantities were overestimated, while bladder was underestimated, by the TG-43 formalism. It was shown that the largest discrepancies in dosimetry between the two calculation methods were due to large prostatic calcifications that attenuated the photon fluence, resulting in significant under-dosage of some prostate volume.

A simple radiobiological model was applied to the patient cohort to study the impact of the two calculations methods on two radiobiological indices: biologically effective dose (BED), and tumor control probability (TCP). It was shown that the current methodology employed in the literature to use this radiobiological model does not account for areas of under-dosage in the prostate as a result of tissue heterogenities and inter-seed effects; inclusion of these effects and the consideration of low-dose regions lead to considerably lower BED and compromised TCP.

A summary of radiation-induced toxicities as a result of the brachytherapy treat- 
ments was also presented where it was concluded that the treatment was well tolerated for most patients. The most common treatment side-effect was minor urinary incontinence as a result of treatment-induced edema, which was resolved shortly after the procedure. Correlations between dosimetric quantities computed with either dose calculation method, and radiation toxicity grades, or clinical endpoints were not drawn, attributing this to the lack of followup data or PSA failures.

Continued efforts in this field of research should focus their attention on the improvement of current post-implant procedures; namely, improved imaging techniques for organ delineation and more complete patient followup data. The usage of dual energy CT, SPECT/CT, or MRI may provide improved organ delineation leading to better certainty in contoured organ volumes ${ }^{80-83}$ and subsequently more accurate patient models. An increase in patient followup period (at least $>5$ years) either through efforts of a multi-center or single institution database, would allow for better outcome studies to draw statistically improved correlations between treatment outcomes and dosimetric, or radiobiological endpoints. Lastly, an improvement to the current model-based dose calculation approach should consider dynamic modeling of prostate edema and seed motion to establish a more accurate model of the entire treatment. 


\section{Appendix A}

\section{Patient Data Storage}

DICOM-RT files for each patient are anonymized, reorganized, and stored in the following file structure:

.../Patients/

Patiento/

DICOM-RT Plan

DICOM-RT Structure

DICOM-RT Dose

CT/

CT Slice 0

CT Slice 1

...

CT Slice $n$

Patient1/

PatientN/

DICOM-RT Plan file: Contains dosimetrist-defined seed center positions, radionuclides used, and air-kerma strength for the brachytherapy treatment.

DICOM-RT Structure file: Contains contour points that define various 
physician-defined organs and treatment volumes.

DICOM-RT Dose file: Contains dose distributions calculated by treatment planning software.

CT files: Contains slice-by-slice information regarding voxel dimensions and CT numbers. 


\section{Appendix B}

\section{Philips Brilliance CT Big Bore Calibration Curve}

The CT calibration curve relates CT numbers (in Hounsfield Units, HU) to electron densities $\left(\mathrm{e}^{-} / \mathrm{cm}^{3}\right)$ relative to water. The conversion of electron densities to mass densities $\left(\mathrm{g} / \mathrm{cm}^{3}\right)$ is done as outlined in the TG-186 report ${ }^{6}$; namely the relative electron density $\rho_{e, \text { rel }}$ can be converted to mass density $\rho$ with maximum error of less than $1 \%$, with the following expression:

$$
\rho=-0.1746+1.176 \rho_{e, r e l}
$$

Table B.1: CT number to mass density calibration curve used for Philips Brilliance CT Big Bore. CT numbers between any points listed are interpolated linearly.

\begin{tabular}{cc}
\hline \hline CT Number [HU] & Mass Density $\left[\mathbf{g} / \mathbf{c m}^{3}\right]$ \\
\hline-1024 & -0.163 \\
-706 & 0.155 \\
-535 & 0.296 \\
-99 & 0.884 \\
10 & 1.001 \\
208 & 1.107 \\
467 & 1.331 \\
1234 & 1.813 \\
3520 & 2.883 \\
5750 & 3.941 \\
8000 & 4.976 \\
160000 & 8.645 \\
\hline \hline
\end{tabular}




\section{Appendix C}

\section{Photon Cross-sections}

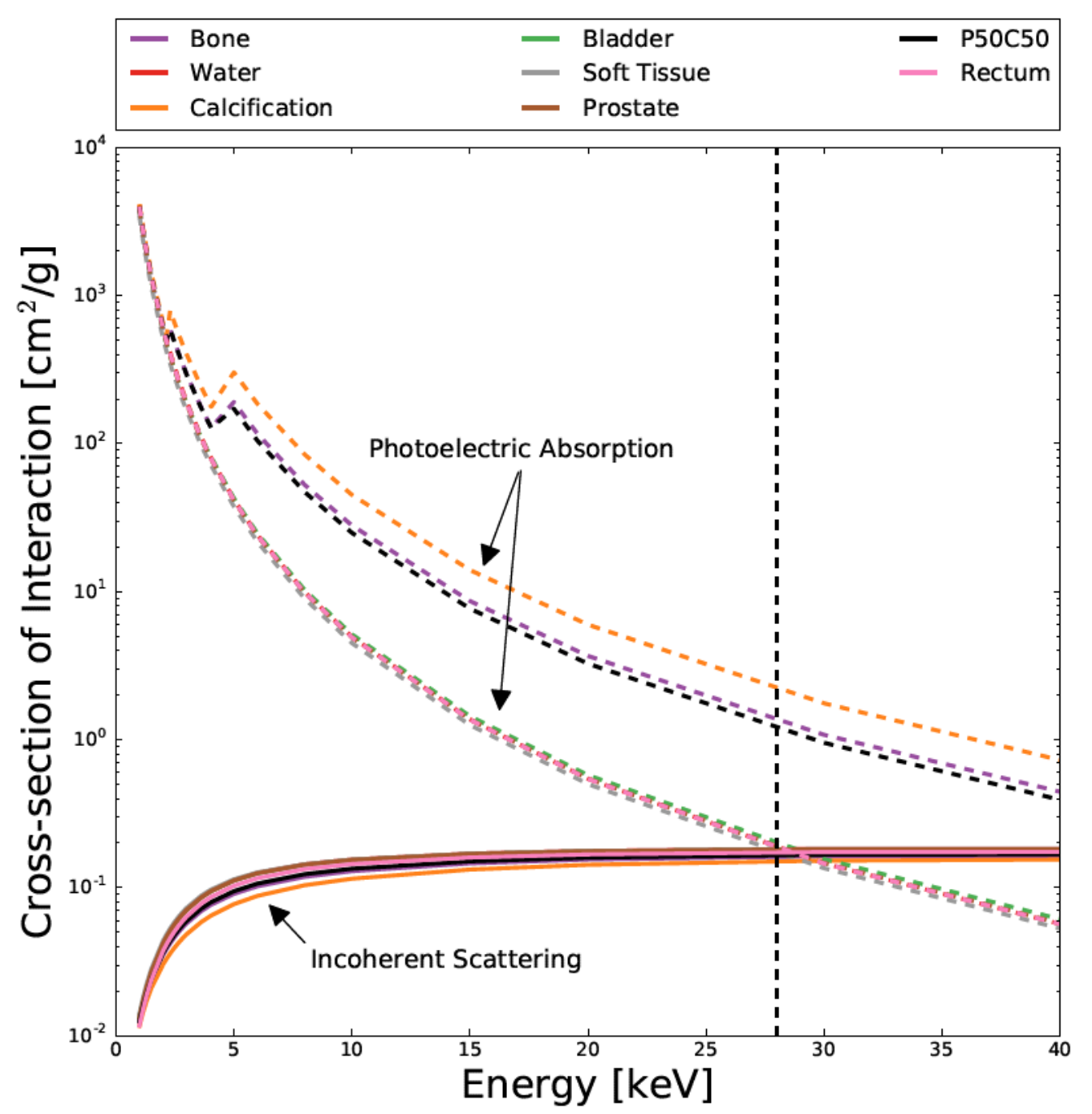

Figure C.1: Photoelectric absorption (dashed lines) and incoherent scattering (solid lines) cross-sections as a function of photon energy of various tissues ${ }^{51}$ on a semilog plot. Dashed vertical line represents average photon energy of ${ }^{125}$ I radioisotope. 


\section{References}

[1] Canadian Cancer Society and National Cancer Institute of Canada. Advisory Committee on Records and Registries. Canadian Cancer Statistics. Canadian Cancer Society, 1987. (p 1)

[2] A. W. Partin, M. W. Kattan, E. N. P. Subong, P. C. Walsh, K. J. Wojno, J. E. Oesterling, P. T. Scardino, and J. D. Pearson. Combination of prostate-specific antigen, clinical stage, and gleason score to predict pathological stage of localized prostate cancer: a multi-institutional update. JAMA, 277(18):1445-1451, 1997. (p 1)

[3] R. Nath, W. S. Bice, W. M. Butler, Z. Chen, A. S. Meigooni, V. Narayana, M. J. Rivard, and Y. Yu. AAPM recommendations on dose prescription and reporting methods for permanent interstitial brachytherapy for prostate cancer: Report of Task Group 137. Med. Phys., 36:5310 - 5322, 2009. (pp 1, 2, 8, and 9)

[4] M. J. Rivard, B. M. Coursey, L. A. DeWerd, M. S. Huq, G. S. Ibbott, M. G. Mitch, R. Nath, and J. F. Williamson. Update of AAPM Task Group No. 43 Report: A revised AAPM protocol for brachytherapy dose calculations. Med. Phys., 31: $633-674,2004 .($ pp 2, 3, and 21)

[5] F. H. Attix. Introduction to Radiological Physics and Radiation Dosimetry. Wiley, New York, 1986. (p 4)

[6] L. Beaulieu et al. Report of the Task Group 186 on model-based dose calcu- 
lation methods in brachytherapy beyond the TG- 43 formalism: Current status and recommendations for clinical implementation. Med. Phys., 39:6208 - 6236, 2012. (pp 4, 21, 23, 59, 66, and 74)

[7] A. K. Carlsson and A. Ahnesjö. The collapsed cone superposition algorithm applied to scatter dose calculations in brachytherapy. Med. Phys., 27:2320 2332, 2000. (p 4)

[8] A. K. Carlsson and A. Ahnesjö. Point kernels and superposition methods for scatter dose calculations in brachytherapy. Phys. Med. Biol., 45:357-382, 2000. message

[9] A. K. Carlsson and A. Ahnesjö. Accounting for high Z shields in brachytherapy using collapsed cone superposition for scatter dose calculation. Med. Phys., 30: 2206-2217, 2003. (p 5)

[10] A. K. Carlsson and A. Ahnesjö. Optimization of the computational efficiency of a 3d, collapsed cone dose calculation algorithm for brachytherapy. Med. Phys., 35(4):1611-1618, 2008. (p 4)

[11] C. Zhou and F. Inanc. Integral-transport-based deterministic brachytherapy dose calculations. Physics in Medicine and Biology, 48(1):73, 2002. (p 4)

[12] G. M. Daskalov, R. S. Baker, D. W. O. Rogers, and J. F. Williamson. Multigroup discrete ordinates modeling of $125 \mathrm{i} 6702$ seed dose distributions using a broad energy-group cross section representation. Med. Phys., 29(2):113-124, 2002. (p 4)

[13] O. Chibani and J. F. Williamson. MCPI: A sub-minute Monte Carlo dose calculation engine for prostate implants. Med. Phys., 32:3688-3698, 2005. ( $p$ 4) 
[14] R. E. P. Taylor, G. Yegin, and D. W. O. Rogers. Benchmarking BrachyDose: Voxel-based EGSnrc Monte Carlo calculations of TG-43 dosimetry parameters. Med. Phys., 34:445 - 457, 2007. (pp 6 and 21)

[15] R. M. Thomson, G. Yegin, R. E. P. Taylor, J. G. H. Sutherland, and D. W. O. Rogers. Fast Monte Carlo dose calculations for brachytherapy with BrachyDose. Med. Phys., 37:(abstract) 3910 - 3911, 2010. (p 4)

[16] J. E. O'Connor. The variation of scattered x-rays with density in an irradiated body. Physics in Medicine and Biology, 1(4):352, 1957. (p 5)

[17] M. J. Rivard, J. L. M. Venselaar, and L. Beaulieu. The evolution of brachytherapy treatment planning. Med. Phys., 36:2136 - 2153, 2009. (p 5)

[18] G. M. Daskalov, R. S. Baker, D. W. O. Rogers, and J. F. Williamson. Dosimetric modeling of the MicroSelectron high-dose rate ${ }^{192}$ Ir source by the multigroup discrete ordinates method. Med. Phys., 27(10):2307-2319, 2000. (p 5)

[19] C. Börgers. Complexity of Monte Carlo and deterministic dose-calculation methods. Physics in Medicine and Biology, 43(3):517, 1998. (p 5)

[20] H. Q. Woodard and D. R. White. The composition of body tissues. Brit. J. Radiol., 59:1209-1219, 1986. (pp 5, 20, and 58)

[21] ICRP. Report of Task Group on Reference Man. ICRP Report 23, ICRP, Washington D.C., 1975. (pp 20 and 58)

[22] ICRU. Photon, electron, photon and neutron interaction data for body tissues. ICRU Report 46, ICRU, Washington D.C., 1992. (pp 5, 20, and 58)

[23] J. F. Carrier, L. Beaulieu, F. Therriault-Proulx, and R. Roy. Impact of interseed attenuation and tissue composition for permanent prostate implants. Med. Phys., 33:595 - 604, 2006. (pp 5, 58, 59, 60, and 62) 
[24] J. F. Carrier, M. D’Amours, F. Verhaegen, B. Reniers, A. G. Martin, E. Vigneault, and L. Beaulieu. Postimplant dosimetry using a Monte Carlo dose calculation engine: a new clinical standard. Int. J. Radiat. Oncol. Biol. Phys., 68:1190 1198, 2007. (pp 5, 59, 60, 64, and 65)

[25] C. A. C. Fekete, M. Plamondon, A. G. Martin, E. Vigneault, F. Verhaegen, and L. Beaulieu. Calcifications in low-dose rate prostate seed brachytherapy treatment: Post-planning dosimetry and predictive factors. Radiotherapy and Oncology, 114(3):339-344, 2015. (pp 5, 58, 60, 61, 62, and 63)

[26] O. Chibani, J. F. Williamson, and D. Todor. Dosimetric effects of seed anisotropy and interseed attenuation for ${ }^{103} \mathrm{Pd}$ and ${ }^{125} \mathrm{I}$ prostate implants. Med. Phys., 32: $2557-2566,2005$. (pp 5 and 59)

[27] N. Miksys, L. Beaulieu, and R. M. Thomson. Monte Carlo dose calculations for permanent prostate brachytherapy: Calcification modelling schemes and sensitivity to tissue compositions. Brachytherapy, 15:S33-S34, 2016. (p 6)

[28] J. Mason, B. Al-Qaisieh, P. Bownes, A. Henry, and D. Thwaites. Investigation of interseed attenuation and tissue composition effects in ${ }^{125} \mathrm{I}$ seed implant prostate brachytherapy. Brachytherapy, 13(6):603-610, 2014. (pp 6, 58, 61, 63, and 64)

[29] I. Kawrakow. Accurate condensed history Monte Carlo simulation of electron transport. I. EGSnrc, the new EGS4 version. Med. Phys., 27:485 - 498, 2000. (p 6)

[30] I. Kawrakow and D. W. O. Rogers. The EGSnrc Code System: Monte Carlo simulation of electron and photon transport. Technical Report PIRS-701, National Research Council Canada, Ottawa, Canada, 2000. (p 6)

[31] G. Yegin. A new approach to geometry modeling for Monte Carlo particle transport: An application to the EGS code system. Nuclear Instruments and Methods 
in Physics Research Section B: Beam Interactions with Materials and Atoms, 211(3):331-338, 2003. (p 6)

[32] R. E. P. Taylor and D. W. O. Rogers. An EGSnrc Monte Carlo-calculated database of TG-43 parameters. Med. Phys., 35:4228 - 4241, 2008. (pp 6 and 30)

[33] B. R. B. Walters, I. Kawrakow, and D. W. O. Rogers. History by history statistical estimators in the BEAM code system. Med. Phys., 29:2745 - 2752, 2002. (p 7)

[34] J. F. Fowler. The linear-quadratic formula and progress in fractionated radiotherapy. Brit. J. Radiol., 62(740):679 - 694, 1989. (p 7)

[35] R. G. Dale and B. Jones. The clinical radiobiology of brachytherapy. The British Journal of Radiology, 71(845):465-483, 1998. (pp 8 and 67)

[36] R. G. Dale. The application of the linear-quadratic dose-effect equation to fractionated and protracted radiotherapy. Int. J. Radiat. Oncol. Biol. Phys., 58(690): $515-528,1985 .(p 8)$

[37] H. D. Thames. An incomplete-repair model for survival after fractionated and continuous irradiations. International Journal of Radiation Biology and Related Studies in Physics, Chemistry and Medicine, 47(3):319-339, 1985. (p 8)

[38] C. C. Ling, J. Roy, N. Sahoo, K. Wallner, and L. Anderson. Quantifying the effect of dose inhomogeneity in brachytherapy: application to permanent prostatic implant with ${ }^{125}$ I seeds. (pp 9 and 23)

[39] R. G. Dale, I. P. Coles, C. Deehan, and J. A. O'Danoghue. Calculation of integrated biological response in brachytherapy. International Journal of Radiation Oncology Biology Physics, 38:633-642, 1997. (p 9) 
[40] X. A. Li, J. Z. Wang, R. D. Stewart, and S. J. DiBiase. Dose escalation in permanent brachytherapy for prostate cancer: Dosimetric and biological considerations. Physics in Medicine and Biology, 48(17):2753, 2003. (p 9)

[41] S. L. Tucker, H. D. Thames, and J. M. G. Taylor. How well is the probability of tumor cure after fractionated irradiation described by Poisson statistics? Radiation Research, 124(3):273-282, 1990. (p 9)

[42] M. Zaider and G. N. Minerbo. Tumour control probability: A formulation applicable to any temporal protocol of dose delivery. Physics in Medicine and Biology, 45(2):279, 2000. (p 9)

[43] S. L. Tucker and J. M. G. Taylor. Improved models of tumour cure. International Journal of Radiation Biology, 70(5):539-553, 1996. ( $p$ 9)

[44] C. Xu, F. Verhaegen, D. Laurendeau, S. A. Enger, and L. Beaulieu. An algorithm for efficient metal artifact reductions in permanent seed implants. Med. Phys., $38: 47-56,2011$. ( p 13)

[45] J. G. H. Sutherland, K. M. Furutani, Y. I. Garces, and R. M. Thomson. Modelbased dose calculations for ${ }^{125}$ l lung brachytherapy. Med. Phys., 39:4365-4377, 2012. (p 13)

[46] J. G. H. Sutherland, N. Miksys, K. M. Furutani, and R. M. Thomson. Metallic artifact mitigation and organ-constrained tissue assignment for Monte Carlo of permanent implant lung brachytherapy. Med. Phys., 41:011712, 2014. (p 13)

[47] N. Miksys, C. Xu, L. Beaulieu, and R. M. Thomson. Development of virtual patient models for permanent implant brachytherapy Monte Carlo dose calculations: Interdependence of CT image artifact mitigation and tissue assignment. Physics in Medicine and Biology, 60(15):6039, 2015. (p 13) 
[48] G. Barequet and M. Sharir. Piecewise-linear interpolation between polygonal slices. In Proceedings of the tenth annual symposium on Computational Geometry, pages 93-102. ACM, 1994. ( $p$ 17)

[49] G. Barequet, M. T. Goodrich, A. Levi-Steiner, and D. Steiner. Contour interpolation by straight skeletons. Graphical Models, 66(4):245-260, 2004. message

[50] A. B. Ekoule, F. C. Peyrin, and C. L. Odet. A triangulation algorithm from arbitrary shaped multiple planar contours. ACM Transactions on Graphics (TOG), 10(2): 182-199, 1991. (p 17)

[51] M. J. Berger, J. H. Hubbell, S. M. Seltzer, J. Chang, J. S. Coursey, R. Sukumar, D. S. Zucker, and K. Olsen. XCOM: Photon cross section database (version 1.5). Technical report, NIST, Gaithersburg, MD, http://physics.nist.gov/xcom, 2010. (pp 19, 21, 23, and 75)

[52] I. J. Chetty et al. Report of the AAPM Task Group No. 105: Issues associated with clinical implementation of Monte Carlo-based photon and electron external beam treatment planning. Med. Phys., 34:4818-4853, 2007. (pp 21 and 23)

[53] C. R. King, T. A. DiPetrillo, and D. E. Wazer. Optimal radiotherapy for prostate cancer: Predictions for conventional external beam, IMRT, and brachytherapy from radiobiologic models. International Journal of Radiation Oncology Biology Physics, 46(1):165-172, 2000. (pp 24 and 49)

[54] R. Nath, W. S. Bice, W. M. Butler, Z. Chen, A. S. Meigooni, V. Narayana, M. J. Rivard, and Y. Yu. AAPM recommendations on dose prescription and reporting methods for permanent interstitial brachytherapy for prostate cancer: Report of Task Group 137. Med. Phys., 36(11):5310-5322, 2009. (pp 25, 66, and 67)

[55] D. J. Carlson, R. D. Stewart, X. A. Li, K. Jennings, J. Z. Wang, and M. Guerrero. 
Comparison of in vitro and in vivo $\alpha / \beta$ ratios for prostate cancer. Physics in Medicine and Biology, 49(19):4477, 2004. (p 25)

[56] J. Z. Wang, M. Guerrero, and X. A. Li. How low is the $\alpha / \beta$ ratio for prostate cancer? International Journal of Radiation Oncology Biology Physics, 55(1): 194-203, 2003. (p 25)

[57] J. Z. Wang, X. A. Li, X. Y. Cedric, and S. J. DiBiase. The low $\alpha / \beta$ ratio for prostate cancer: What does the clinical outcome of HDR brachytherapy tell us? International Journal of Radiation Oncology Biology Physics, 57(4):1101-1108, 2003. (p 25)

[58] J. Z. Wang, N. A. Mayr, S. Nag, J. Montebello, N. Gupta, N. Samsami, and C. Kanellitsas. Effect of edema, relative biological effectiveness, and dose heterogeneity on prostate brachytherapy. Med. Phys., 33(4):1025-1032, 2006. (p 25)

[59] US Department of Health and Human Services. National Cancer Institute. Common Terminology Criteria for Adverse Events (CTCAE). Version 4.0. 2009 (v4. 03: June 14, 2010). (p 25)

[60] J. F. Williamson. Comparison of measured and calculated dose rates in water near I-125 and Ir-192 seeds. Med. Phys., 18:776 - 786, 1991. (p 30)

[61] J. Dolan, Z. Li, and J. F. Williamson. Monte Carlo and experimental dosimetry of an ${ }^{125}$ I brachytherapy seed. Med. Phys., 33:4675 - 4684, 2006. (p 30)

[62] G. Landry, B. Reniers, L. Murrer, L. Lutgens, E. Bloemen-Van Gurp, J. P. Pignol, B. Keller, L. Beaulieu, and F. Verhaegen. Sensitivity of low energy brachytherapy Monte Carlo dose calculations to uncertainties in human tissue composition. Med. Phys., 37(10):5188-5198, 2010. (pp 58 and 59) 
[63] H. Afsharpour, M. D’Amours, B. Coté, J.-F. Carrier, F. Verhaegen, and L. Beaulieu. A Monte Carlo study on the effect of seed design on the interseed attenuation in permanent prostate implants. Med. Phys., 35:3671 - 3681, 2008. (pp 58 and 62)

[64] ICRP. Basic Anatomical and Physiological Data for Use in Radiological Protection: Reference Values. Annals of the ICRP 89, ICRP, Washington D.C., 2003. (p 58)

[65] A. R. M. Haidari, N. Miksys, J. E. Cygler, E Choan, G. Perry, O. Holmes, and R. M. Thomson. Effect of tissue heterogeneity and interseed effects on urethral dose for permanent prostate brachytherapy. Brachytherapy, 15:S186-S187, 2016. (p 63)

[66] D. Taussky, L. Austen, A. Toi, I. Yeung, T. Williams, S. Pearson, M. McLean, G. Pond, and J. Crook. Sequential evaluation of prostate edema after permanent seed prostate brachytherapy using CT-MRI fusion. International Journal of Radiation Oncology Biology Physics, 62(4):974-980, 2005. (p 66)

[67] R. Taschereau, J. Roy, and J. Pouliot. Monte Carlo simulations of prostate implants to improve dosimetry and compare planning methods. Med. Phys., 26 (9):1952-1959, 1999. (p 66)

[68] M. Gao, J. Z. Wang, S. Nag, and N. Gupta. Effects of seed migration on postimplant dosimetry of prostate brachytherapy. Med. Phys., 34(2):471-480, 2007. (p 66)

[69] Z. Gao, D. Wilkins, L. Eapen, C. Morash, Y. Wassef, and L. Gerig. A study of prostate delineation referenced against a gold standard created from the Visible Human data. Radiotherapy and Oncology, 85(2):239-246, 2007. (p 66) 
[70] M. J. Ackerman, V. M. Spitzer, A. L. Scherzinger, and D. G. Whitlock. The Visible Human data set: an image resource for anatomical visualization. MEDINFO, 8: 1195-1198, 1994. (p 66)

[71] H. Afsharpour, S. Walsh, C. A. C. Fekete, E. Vigneault, F. Verhaegen, and L. Beaulieu. On the sensitivity of $\alpha / \beta$ prediction to dose calculation methodology in prostate brachytherapy. International Journal of Radiation Oncology* Biology* Physics, 88(2):345-350, 2014. (p 67)

[72] R. G. Stock, N. N. Stone, A. Tabert, C. lannuzzi, and J. K. DeWyngaert. A doseresponse study for ${ }^{125}$ | prostate implants. International Journal of Radiation Oncology ${ }^{*}$ Biology ${ }^{\star}$ Physics, 41(1):101-108, 1998. (p 68)

[73] M. A. Papagikos, P. J. Rossi, D. L. McCullough, P. E. Clark, W. R. Lee, et al. Dosimetric quantifiers for low-dose-rate prostate brachytherapy: Is $\mathrm{V}_{100}$ superior to $D_{90}$ ? Brachytherapy, 4(4):252-258, 2005. (p 68)

[74] L. Potters, C. Morgenstern, E. Calugaru, P. Fearn, A. Jassal, J. Presser, and E. Mullen. 12-year outcomes following permanent prostate brachytherapy in patients with clinically localized prostate cancer. The Journal of Urology, 173 (5):1562-1566, 2005. message

[75] A. V. Taira, G. S. Merrick, R. W. Galbreath, K. E. Wallner, and W. M. Butler. Natural history of clinically staged low-and intermediate-risk prostate cancer treated with monotherapeutic permanent interstitial brachytherapy. International Journal of Radiation Oncology Biology Physics, 76(2):349-354, 2010. message

[76] E. F. Miles, J. W. Nelson, A. K. Alkaissi, S. Das, R. W. Clough, G. Broadwater, M. S. Anscher, J. P. Chino, and J. R. Oleson. Biologically Effective Dose (BED) correlation with biochemical control after low-dose rate prostate brachytherapy 
for clinically low-risk prostate cancer. International Journal of Radiation Oncology Biology Physics, 77(1):139-146, 2010. (p 68)

[77] W. J. Morris, I. Spadinger, M. Keyes, J. Hamm, M. McKenzie, and T. Pickles. Whole prostate $D_{90}$ and $V_{100}$ : A dose-response analysis of 2000 consecutive ${ }^{125}$ I monotherapy patients. Brachytherapy, 13(1):32-41, 2014. (p 68)

[78] A. Yorozu, N. Kuroiwa, A. Takahashi, K. Toya, S. Saito, T. Nishiyama, Y. Yagi, T. Tanaka, Y. Shiraishi, and T. Ohashi. Permanent prostate brachytherapy with or without supplemental external beam radiotherapy as practiced in Japan: Outcomes of 1300 patients. Brachytherapy, 14(2):111-117, 2015. (p 68)

[79] R. G. Stock, N. N. Stone, J. A. Cesaretti, and B. S. Rosenstein. Biologically effective dose values for prostate brachytherapy: Effects on PSA failure and posttreatment biopsy results. International Journal of Radiation Oncology Biology Physics, 64(2):527-533, 2006. (p 68)

[80] B. H. Hasegawa, K. H. Wong, K. Iwata, W. C. Barber, A. B. Hwang, A. E. Sakdinawat, M. Ramaswamy, D. C. Price, and R. A. Hawkins. Dual-modality imaging of cancer with SPECT/CT. Technology in Cancer Research \& Treatment, 1(6): 449-458, 2002. ( p 71)

[81] G. Landry, P. V. Granton, B. Reniers, M. C. Öllers, L. Beaulieu, J. E. Wildberger, and F. Verhaegen. Simulation study on potential accuracy gains from dual energy CT tissue segmentation for low-energy brachytherapy Monte Carlo dose calculations. Physics in Medicine and Biology, 56(19):6257, 2011. message

[82] J. J. Prete, B. R. Prestidge, W. S. Bice, D. F. Dubois, and L. A. Hotchkiss. Comparison of MRI- and CT-based post-implant dosimetric analysis of transperineal interstitial permanent prostate brachytherapy. Radiation Oncology Investigations, 6(2):90-96, 1998. message 
[83] S. Nag, W. Bice, K. DeWyngaert, B. Prestidge, R. Stock, and Y. Yu. The American Brachytherapy Society recommendations for permanent prostate brachytherapy postimplant dosimetric analysis. International Journal of Radiation Oncology Biology Physics, 46(1):221-230, 2000. (p 71) 